\title{
The Controversy on the Inverse-Square Law for Coulomb's Interactions
}

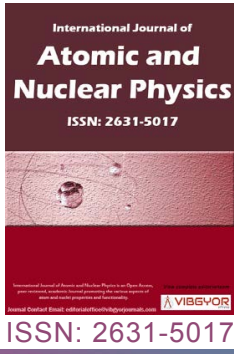

\section{Wladimir Guglinski*}

Escola de Engenharia da Universidade Federal de Minas Gerais, Brazil

\begin{abstract}
Abdus Salam and his co-workers proposed the concept of strong gravity in the 1960 s, as an alternative to the young $Q C D$, so that to solve the puzzles concerning to confinement and asymptotic freedom, not requiring, as occurs in $Q C D$, to abandon the behavior of a force acting from the inverse-square law. At that time asymptotic freedom in QED was observed by some theorists, and by Gerard't Hooft in 1972, whose physical significance however was realized only one year later by David Gross, Frank Wilczek and David Politzer. They "rehabilitated" the Quantum Field Theory, because prior to their discovery it was under suspicion, since Coulomb interactions become infinitely strong at very short distances. But this approach has not so far led to a Grand Unified Theory. Then we are forced to think whether the "rehabilitation" is possible by other alternative, rather than by asymptotic freedom, because there are so many unacceptable puzzles in nuclear physics, that they oblige us to conclude that some of the fundamental principles of the nuclear theory are wrong. Therefore, if some principles of the nuclear theory are wrong, it is possible that the "rehabilitation" must be sought in the atomic nucleus, as proposed herein.
\end{abstract}

\section{Keywords}

Nuclear magnetic moment, Electric quadruopole moment, Excited even Z = N nuclei, Transition probability between states, Asymptotic freedom, Coulomb's law, Weak interactions

\section{Introduction}

Along the last 15 years, several new experimental findings come, year after year, demolishing more and more the pillars on which the theoretical structure of the current Nuclear Physics was erected. The hypothesis of the strong nuclear force is one among the pillars. Other pillar is the old Coulomb's Law, since the stability of atomic nuclei is attributed to the successful interaction by strong force between protons and neutrons, able to win the Coulomb repulsion.

The first person to note that Coulomb's Law seems to prevail in the distances of few femtometers, inside the atomic nuclei, was Rutherford. His experiment, where alpha particles hit the nucleus U238, suggested him that up to the distance of $30 \mathrm{fm}$ the repulsion between $\mathrm{U} 238$ and the alpha particle follows the old Coulomb's law. Nevertheless, Rutherford (and all the theorists who after him dealt with that question), did not take in consider-

* Corresponding author: Wladimir Guglinski, Escola de Engenharia da Universidade Federal de Minas Gerais, Av.Presidente Antonio Carlos, 6627, Pampulha, Belo Horizonte-MG, Brazil

Accepted: August 19, 2019; Published: August 21, 2019

Copyright: (C) 2019 Guglinski W. This is an open-access article distributed under the terms of the Creative Commons Attribution License, which permits unrestricted use, distribution, and reproduction in any medium, provided the original author and source are credited.

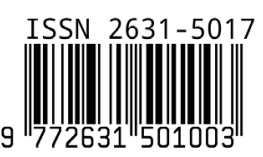

Guglinski. Int J At Nucl Phys 2019, 4:013 
ation that the velocity of the alpha particle, which is $10 \%$ of the light speed, might have influence in his experiment, because the theorists never before did consider that the structure of the electric charges, of the elementary particles, can be composed by fermions which fill the quantum vacuum, in spite of the idea that quantum vacuum is filled by fermions is not a novelty in theoretical physics [1]. If the electric field is composed by fermions that move with the speed $c$ of light along straight strings which leave the body of protons, electrons, quarks, and any charged particle, then the interaction between the strings of two protons, moving with relative velocity $V=0.1$.c one against the other, the energy of interaction will be proportional to $(c+V)^{2} / c^{2}=1.21$. And if one of the protons moves away the other one with velocity $v=0.1 . c$, the interaction energy is proportional to $(\mathrm{c}-\mathrm{V})^{2} / \mathrm{c}^{2}=0.81$, and so the difference of energy interaction between the two electric fields in the two situations is 1.49. Therefore, there is chance to reach to equations from which Rutherford's experiments can be interpreted by a new approach, and perhaps to develop a new theory free of unacceptable paradoxes.

In this paper are exhibited striking evidences, supported by mathematical calculations, testifying against the hypothesis of the strong nuclear force. And the evidences are reinforced by the new experimental findings, along the last 15 years, demolishing the pillars that sustain the current Nuclear Theory.

As the strong nuclear force is a pillar of the Nuclear Physics, the acceptation by nuclear theorists, that there is not any strong nuclear force playing any role inside the atomic nuclei, will bring repercussions not only in the field of Nuclear Physics, but also for the field of Particle Physics, because the inexistence of the strong force implies that Coulomb's Law decreases for distances in the range of few femtometers inside the atomic nuclei, as shown in the present paper. And thereby, for the "rehabilitation" of the Quantum Field Theory, there is no need to consider the hypothesis of asymptotic freedom proposed by Gross, Wilczek and Politzer. So, new pillars for the Theoretical Physics can emerge from the acceptation, by the nuclear theorists, that protons and neutrons are not bound by strong nuclear force inside the atomic nuclei. The destiny of Theoretical Physics is in their hands, and they cannot face their responsibility with negligence, because is a nonsense to sacrifice the scientific advancement for feeling that their pride was hurt by having for decades embarked on a wrong path.

\section{Gamow's Theory Cannot be Correct}

Alpha particle is emitted by radioactive nuclei as uranium, thorium, and polonium. But its emission is not authorized by the laws of classical physics. For instance, in $\mathrm{U} 238$ the $2 \mathrm{He} 4$ is confined into a potential due to the strong nuclear force, which is a little larger than the Coulomb repulsion on the alpha particle. By considering the classical physics, the alpha particle cannot leave the U238. So, the alpha decay of U238 violates the classical physics, and that's why Gamow proposed his theory, according to which the alpha particle can be emitted through the tunneling effect. Let us analyze his theory:

a) Suppose that due to the quantum tunneling the alpha particle succeeds to cross the difference of energy between the strong nuclear potential and the Coulomb potential.

b) The strong nuclear force has a very short range of interaction, actuates at distances shorter than $3.0 \mathrm{fm}$ only. The radius of Th234 (resulted from the U238 decay) is about $7.0 \mathrm{fm}$, and the radius of $2 \mathrm{He} 4$ is about $2.0 \mathrm{fm}$. So, when the alpha particle reaches the point where the distance " $d$ " (between Th234 and 2He4) is $d=$ $12 \mathrm{fm}$, there is no longer strong nuclear force acting on the particle. Therefore, the quantum tunneling effect is over. As consequence now we have to analyze what occurs with the alpha particle independently of the Gamow's theory on the quantum tunneling, because there is no longer any quantum tunneling effect on the alpha particle.

c) At the point where $d=12 \mathrm{fm}$ finished the attraction between Th234 and $2 \mathrm{He} 4$, and so for $\mathrm{d}>12 \mathrm{fm}$ only the Coulomb repulsion is acting on the alpha particle. The potential Coulomb repulsion between Th234 and $2 \mathrm{He} 4$, in that point with $d=12 \mathrm{fm}$, is about $27 \mathrm{MeV}$.

d) As at the point with $d=12 \mathrm{fm}$ only the Coulomb repulsion is acting on the alpha particle, and the potential of repulsion is $27 \mathrm{MeV}$, then the alpha particle is submitted to acceleration. And when it arrives to the point where is null the potential energy due to the repulsion with the Th234, its kinetic energy must be near to 27 
$\mathrm{MeV}$, since the potential energy of $27 \mathrm{MeV}$ was converted to kinetic energy. The energy lost due to acceleration of the charge of alpha particle is about $0.5 \mathrm{MeV}$.

e) Nevertheless, when the alpha particle leaves the Th234 and reaches the point where its repulsion with Th234 is practically null, its kinetic energy is only 4.2 MeV. So, Gamow's theory creates a paradox.

\section{Conclusions}

1. First of all, note that Gamow's paradox has nothing to do with quantum tunneling, because the paradox lies in the fact that his theory cannot explain why the alpha particle does not leave Th234 with kinetic energy $27 \mathrm{MeV}$. His theory is unacceptable, but the invalidity of the theory has no connection with the quantum tunneling phenomenon, because as it was shown here, even if the alpha particle crosses by quantum tunneling the barrier due to the strong nuclear force, it is impossible to explain why the alpha particle does not emerge with kinetic energy of $27 \mathrm{MeV}$, as is expected from his theory. His quantum tunneling theory cannot explain why the alpha particle leaves the Th234 with only 4.2 MeV.

2. Gamow proposed his quantum tunneling theory with the aim to explain how the alpha particle is able to cross the barrier due to the strong nuclear force, since it cannot be explained by the classical physics. But note that alpha decay of $U 238$ requires the tunneling effect because:

- By considering any the current nuclear model, it's impossible to find any other cause responsible for the U238 decay. That's why Gamow proposed the quantum tunneling, because there is no possible to find any other cause, by considering the current nuclear models.

- Nevertheless, if one considers the new nuclear model proposed by the author, where a nucleon $2 \mathrm{He} 4$ occupies the center of the atomic nuclei, it's possible to explain the U238 decay without the tunneling effect, as explained ahead.

- Indeed, the emission of alpha particle by U238 is explained in the book Quantum Ring Theory [2], in the Chapter entitled "Contribution of gamma-rays as the cause of alpha decay", in the page 186. The cause of emission of the alpha particle of a nucleus $\mathrm{U} 238$ is the bombardment of that nucleus by the gamma-rays emitted by the alpha decay of others U238 nuclei in the sample, because the central $2 \mathrm{He} 4$ of the U238 can be hit by a gamma-ray with suitable energy, able to remove it. In the book it is calculated that from such a process the half-life of U238 has the magnitude of $10^{9}$ years, because the central $2 \mathrm{He} 4$ can be removed only when it is hit by a gamma-ray whose direction of motion is coincident with the oscillation of the central $2 \mathrm{He} 4$ along the Z-axis of the U238. Unlike, if we consider the current nuclear models, where there is not a central $2 \mathrm{He} 4$ oscillating along a Z-axis, the half-life of U238 cannot reach the magnitude of billion years, because an alpha particle inside the $\mathrm{U} 238$ can be removed when hit by a gamma-ray moving in any direction. That's why the quantum tunneling is indispensable in the current Nuclear Physics.

- This new nuclear model, with a central $2 \mathrm{He} 4$, survived to a test in which succumbed all the current nuclear models: to calculate the magnetic moment of exotic nuclei. This is shown in a series of three papers published by the author [3-5].

- The Gamow's paradox has its origin in the value $27 \mathrm{MeV}$ of the potential at the distance $d=$ $12 \mathrm{fm}$ from the Th234, because the nuclear theorists adopted the assumption that Coulomb's law continues the same for any distance " $d$ " between two electrically charged particles. But we cannot be sure if such assumption is correct, because whereas the Coulomb's law was inferred from experiments with macroscopic charged bodies separated by large distances, with they both at rest to each other, on another hand in Modern Physics was not adopted any physical model for the electric field, and therefore the microscopic laws that rule the behavior of electric fields were never discovered. By considering a physical model of electric field, composed by fermions of the quantum vacuum, it is possible to show that Coulomb's law has a catastrophic collapse in the distance shorter than $d=30 \mathrm{fm}$, and the paradox why alpha particle leaves the Th234 with kinetic energy 4.2 MeV can be solved, as shown in the paper [5].

- As the nuclear theorists assumed that Coulomb's law continues valid for any distance d, 
they had no other alternative than to propose the existence of the strong nuclear force, which is the second assumption responsible for the Gamow's paradox.

- It's well to emphasize that it's mandatory to consider the tunneling effect (for the explanation on why the alpha particle is able to leave the U238) only "if" the current foundations of the Nuclear Physics are correct. However, such "if" has no chance. And such "if" has no chance (as will be shown in this paper) because several experimental findings are demolishing the fundamental pillars of the theory. By consequence, there is need to look for new foundations, and to adopt a new nuclear model proposed from the new foundations, and the current models must be replaced. From such situation, with the adoption of a new nuclear model where a central $2 \mathrm{He} 4$ occupies the center of all atomic nuclei, the tunneling effect can be discarded as the cause why the alpha particle is able leave the U238. The new nuclear model is born free of Gamow' paradox.

3. As final and unavoidable conclusion, Gamow's theory is wrong and unacceptable.

4. It's impossible to solve the puzzle of U238 alpha decay by keeping the hypothesis that nucleons are bound by strong nuclear force inside the atomic nuclei. And as we will see, the hypothesis of the strong nuclear force is responsible for the introduction of many other paradoxes in $\mathrm{Nu}$ clear Physics.

\section{Clusters in Nuclei}

By considering the current nuclear models, there is no way to know when an alpha particle will succeed to cross the barrier by tunneling effect, in the alpha decay. As emphasized by Heisenberg, the emission in alpha decay is a random phenomenon with no physical cause, because according to Quantum Mechanics some phenomena do not need physical causes for their occurrence. Then let us analyze such assumption.

- Consider a nuclear model composed by protons, neutrons, deuterons, and alpha particles, where those nucleons are bound through the strong nuclear force. The alpha particle is tied to the U238 strongly, because it has four nucleons interacting via strong nuclear force with the other nucleons. Then it is easier for the U238 to emit two protons and two neutrons, one after other, and not to emit an alpha particle. The emission of an alpha particle requires more energy, and therefore the nucleus will not emit it.

- Consider that U238 is composed by deuterons and alpha particles. In this case it is easier for the nucleus to emit two deuterons, instead of one alpha particle.

- Eisberg and Resnick explained this puzzle in their book [6], saying that the emission of the alpha particle is more "comfortable" for the nucleus U238, because it is energetically more favorable for the stability of the daughter nucleus, and so we conclude that the alpha particle decides to leave the U238 because she follows the principles of ethics, and prefers by herself to undertake her very hard task of leaving the nucleus, instead of to leave to protons or deuterons a task which is easier to them. In resume, the alpha particle prefers to violate the Last Action Principle, and to follow the laws of ethics.

- Of course the argument cited in Eisberg-Resnick book is very strange. Besides, the discovery that heavy nuclei have alpha decay suggested to the nuclear theorists that clusters of nucleons might be formed inside the atomic nuclei. Then consider that U238 is formed by clusters of alpha particles only. In this case it would be possible to explain the emission of alpha particles, since there were no protons, neutrons, neither deuterons available to be emitted easier than the alpha particle. However, in 2008 researchers at the Thomas Jefferson National Accelerator have discovered that within the nuclei the protons have 20 times more chance to pair up with neutrons than with other protons. (Source: Thomas Jefferson National Accelerator Facility, published online by the journal Science, at the Science Express website). Therefore, since 2008 it is known that the nuclei are not composed by clusters of alpha particles, and so the experiment made in Jef Lab demolished the hypothesis that alpha particle can be emitted by U238 following random laws, as supposed by Heisenberg, if we consider that the strong nuclear force is really responsible for the aggregation of nucleons inside the atomic nuclei. 


\section{Solution for the Unacceptable Gamow's Paradox}

The paradox is solved by considering a new Coulomb's Law for distances shorter than Bohr's radius, as shown in the author's paper [5], where are calculated the magnetic moments for the isotopes of iron, but also it is shown that Gamow's paradox can be solved by adopting a new Coulomb's Law, where the electric fields of the elementary particles are composed by the fermions that compose the quantum vacuum [1]. Then the energy interaction between two electrically charged particles $A$ and $B$ depends on:

- The relative velocity $V_{R}$ of interaction between the fermions of the quantum vacuum that compose the two electric fields of the particles $A$ and $B$. As in each electric field the fermions move with the velocity $c$ of light, then the velocity $V_{R}$ depends on the relative velocity $\mathrm{V}$ between the particles $A$ and $B$, and thereby:

- If $A$ is at rest and $B$ moves toward $A$ with velocity $V$, the energy interaction increases with the growth of $\mathrm{V}$, and therefore it's larger than that considered in the conventional Coulomb's Law, where the energy interaction does not depend on the velocity.

- If $A$ is at rest and $B$ moves with velocity $V$ increasing the distance with $A$, the energy interaction is smaller than that considered in the conventional Coulomb's Law, and the decrease of energy depends on $\mathrm{V}$.

- If $A$ moves with velocity $v$ and $B$ moves with velocity $V$ toward $A$, the energy interaction is larger than that considered in the conventional Coulomb's Law, and the growth of energy depends on $v$ and $V$.
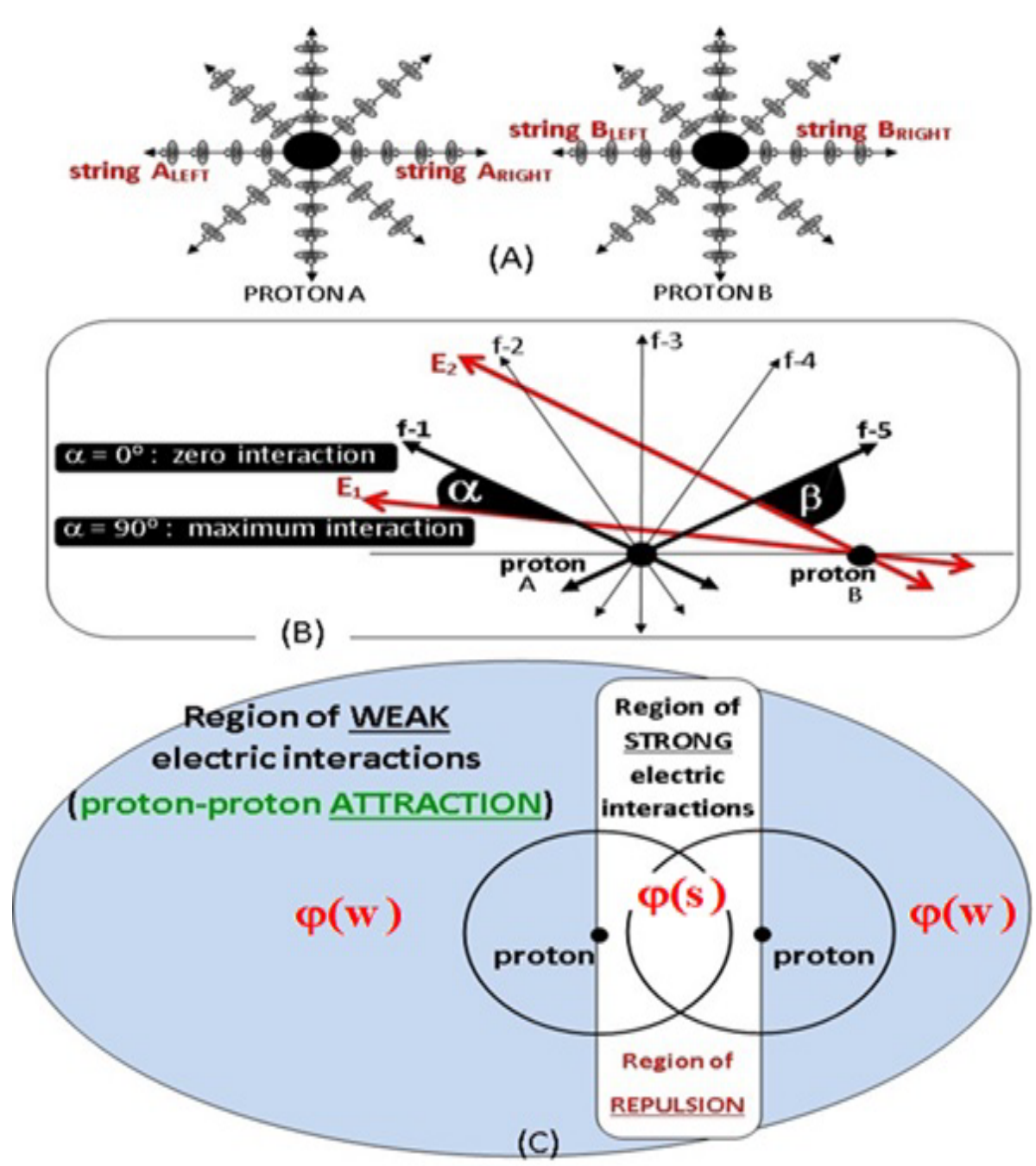

Figure 1: There are four types of Coulomb's interaction between two protons A and B, as proposed in [5], from which, as shown ahead, Gamow's paradox can be solved without introduction of any new paradox. 
A resume of the calculations in [5], concerning the Gamow's paradox, is exhibited ahead.

- Influence of electric field composed by electricitons in the emission of alpha particle by U238

The basic mechanisms that rules the electric interactions through the New Coulomb's Law was firstly proposed in [7], later improved in [5], and proposed as final version in [8]. The structure of the electric field composed by fermions of the quantum vacuum, proposed in $[5,8]$, is seen here in the Figure 1. Ahead is explained how the motion of a proton influences the behavior of the fermions of the quantum vacuum that composes its electric field, when two protons have Coulomb interaction. The fermions of the quantum vacuum which have electric charges were baptized as "electricitons" in the author's theory.

As proposed in [5] and seen in the Figure 1A, the electricitons move with the velocity $c$ of light, along strings leaving the body of the proton. For a distance $\mathrm{d} \geq 10^{-11} \mathrm{~m}$, the interaction between two particles occur through the old Coulomb's Law, as proposed in [5], and confirmed in [8]. Ahead is proposed the parameter $\Delta(\mathrm{sw})$, which relates the energy interaction between electricitons of the electric fields of two particles, when the distance between them is $\mathrm{d} \leq 10^{-11} \mathrm{~m}$, as follows.

\section{a) Two particles at rest}

For two particles $A$ and $B$ at rest, the intensity of interaction between electricitons of the strings $A_{\text {RIGHT }}$ and $B_{\text {LEFT }}$, seen in Figure $1 A$, given by the parameter $\Delta(\mathrm{ws})$, is:

$$
\Delta(\mathrm{sw})=\mathrm{C}^{\left(1+\frac{\mathrm{d}}{\mathrm{w}}\right)}
$$

where

- $\mathrm{c}$ is the speed of light

- $10^{-15}<d<10^{-11}, \mathrm{~d}$ is the distance between the particles.

- $\Phi=10^{-11}$

$$
\Delta(\mathrm{sw})=\mathrm{C}^{\left(\Psi+\frac{\mathrm{d}}{\Phi}\right)}
$$

where

- $10^{-16}<d \leq 10^{-15}$

- $\Phi=10^{-11}$

- $\Psi=1-\mathrm{a}$, where:

$a=0.9$ for $d=0.9 \times 10^{-15} ; a=0.8$ for $d=0.8 \times 10^{-15}$; $\ldots a=0.1$ for $d=0.1 \times 10^{-15}$ b) One particle moving with speed V toward other particle at rest

For a particle $A$ moving with velocity $V$ toward a particle $B$ at rest, as occurs for the alpha particle emitted by Po212 interacting with the U238, the intensity of interaction between electricitons of the strings $A_{\text {RIGHT }}$ and $B_{\text {LEFT }}$ given by the parameter $\Delta(w s)$, is

$$
\Delta(\mathrm{sw})=\left[\left(\mathrm{c}+\left(\frac{\mathrm{Q}}{\mathrm{e}}\right) \cdot \mathrm{V}\right)^{2}\right]^{\left(1+\frac{\mathrm{d}}{\mathrm{\phi}}\right)}
$$

$Q$ is the charge of the particle, in Coulomb, and $\mathrm{e}=1.6 \times 10^{-19} \mathrm{C}$

\section{c) One particle moving with speed $\mathrm{V}$ leaving a nucleus}

For a particle leaving with velocity $\mathrm{V}$ a nucleus at rest, as occurs in the U238 beta-decay, the intensity of interaction between electricitons of the strings $A_{\text {RIGHT }}$ and $B_{\text {LEFT' }}$ given by the parameter $\Delta(w s)$, is:

$$
\Delta(\mathrm{sw})=\left[\left(\mathrm{c}-\left(\frac{\mathrm{Q}}{\mathrm{e}}\right) \cdot \mathrm{V}\right)^{2}\right]^{\left(1+\frac{\mathrm{d}}{\mathrm{w}}\right)}
$$

d) Two particles moving with speed $V$ and $v$ in scattering experiments

For a proton $\mathrm{A}$ moving with velocity $\mathrm{V}$ toward $\mathrm{a}$ particle $B$ with velocity $v$ in scattering experiments, the intensity of interaction between electricitons of the strings $A_{\text {RIGHT }}$ and $B_{\text {LEFT' }}$ given by the parameter $\Delta(w s)$, is:

$$
\Delta(\mathrm{sw})=\left[\left(\mathrm{C}+\left[\frac{\mathrm{Q}+\mathrm{q}}{\mathrm{e}}\right] \cdot(\mathrm{V}+\mathrm{v})^{2}\right)\right]^{\left(1+\frac{\mathrm{d}}{\mathrm{\phi}}\right)}
$$

$\mathrm{Q}$ and $\mathrm{q}$ are the charges of the particles, in Coulomb, and e $=1.6 \times 10^{-19} \mathrm{C}$

- Solution for the Gamow's first paradox, in alpha decay of U238

Let us compare Rutherford's experiment with the alpha decay in U238.

\section{a) Rutherford scattering experiment}

Alpha particles with $8.8 \mathrm{MeV}$ move with $2.91 \times$ $10^{7} \mathrm{~m} / \mathrm{s}$, when exit Po212. Rutherford discovered from his experiment that up to the distance $d=3 \times$ $10^{-14} \mathrm{~m}$ the interaction of the alpha particle with the nucleus U238 was according to Coulomb's law, and for his calculation obviously he did not consider 


\begin{tabular}{|c|c|c|c|c|c|c|c|}
\hline 4 & A & B & c & D & E & $\mathbf{F}$ & G \\
\hline 1 & & & \multicolumn{2}{|c|}{$C 3^{\wedge}(1+(E 3 / D 3))$} & & & \\
\hline 2 & NEW COULOMB LAW & & c & $\Phi$ & d & V & $\mathbf{v}$ \\
\hline 3 & particles at rest & $9 E+16$ & $3 E+08$ & $1 \mathrm{E}-11$ & $1 E-11$ & & \\
\hline 4 & one particle moving toward targe & $1,444 E+17$ & $3 E+08$ & $1 \mathrm{E}-11$ & $3 E-14$ & $2,9 E+07$ & \\
\hline 5 & one particle leaving a nucleus & $7,593 E+16$ & $3 E+08$ & $1 \mathrm{E}-11$ & $3 E-14$ & $2 E+07$ & \\
\hline 6 & scaterring experiments & $7,61 \mathrm{E}+17$ & $3 \mathrm{E}+08$ & $1 \mathrm{E}-11$ & $3 E-14$ & $2,9 E+08$ & $2,9 E+08$ \\
\hline 7 & & & & & & & \\
\hline 8 & OLD COULOMB LAM & used & by $\mathbf{R}$ & erfo & & & \\
\hline 9 & particles at rest & $1,012 E+17$ & $3 E+08$ & $1 \mathrm{E}-11$ & $3 E-14$ & & \\
\hline 10 & & & $\operatorname{con}^{\wedge}(1+1$ & D9)/^2 & & & \\
\hline
\end{tabular}

Figure 2: Rutherford scattering experiment and alpha particle emission by U238, both interpreted from the New Coulomb Law.

the velocity of the alpha particle regarding the nucleus U238, because in the current theories the Coulomb's law does not take in consideration the velocity of the particles. From the eq. (1), the $\Delta(\mathrm{sw})$ factor, in the distance $3 \times 10^{-14} \mathrm{~m}$, is:

$$
\Delta(\mathrm{sw})=\mathrm{C}^{\left(1+\frac{d}{\Phi}\right)}=\left[3 \times 10^{8}\right]^{\left[1+\left(\frac{10^{-11}}{10^{-11}}\right)\right]}=9 \times 10^{16}
$$

This result is seen in the cell B3 of the calculus made by Excel, in the Figure 2, and the obtained value $9 \times 10^{16}$ concerns to the new Coulomb law. In the cell E3 it is seen that the distance considered is $\mathrm{d}=1 \times 10^{-11} \mathrm{~m}$ (and not $\mathrm{d}=3 \times 10^{-14} \mathrm{~m}$, which was considered by Rutherford in his calculations). Actually eq. (1) cannot be applied, to be compared with the Rutherford calculus, because he used the old Coulomb law, and eq. (1) is referred to the new law.

The correct equation to be compared to his calculus is the eq. (7), where it is considered the same $d=3 \times 10^{-14} \mathrm{~m}$ used by Rutherford.

$\Delta(\mathrm{sw})=\mathrm{C}^{(1+\mathrm{d} / \Phi) 2}=\left[3 \times 10^{8}\right]^{\left[1+3.10^{-14} / 10^{-11}\right]^{2}}=1.012 \times 10^{17}$

In eq. (7) the distance $d=3 \times 10^{-14} \mathrm{~m}$ is seen in the cell E9 of Figure 2. In the Rutherford experiment we have to calculate the value of the $\Delta(\mathrm{sw})$ factor from the eq. (3), and to compare it with the result of eq. (7). For the alpha particle, $Q=2$.e, and then:

$\Delta(\mathrm{sw})=\left[\left(\mathrm{C}+\left(\frac{\mathrm{Q}}{\mathrm{e}}\right) \cdot \mathrm{V}\right)^{2}\right]^{2\left(1+\frac{4}{6}\right)}=\left[\left(3 \times 10^{8}+2 \times 2.91 \times 10^{7}\right)^{2}\right]^{\left(1+\frac{3.10^{-14}}{10^{-1}}\right)}=1.44 \times 10^{17}$
As seen, the value $1.44 \times 10^{17}$ is close to 1.012 $\times 10^{17}$, justifying the Rutherford interpretation that the alpha particle emitted by the Po212 followed the Coulomb's law up to the distance $d=3 \times 10^{-14} \mathrm{~m}$ in the scattering with the U238.

According to eq. (3), the curve for the Rutherford experiment is shown in the Figure 3.

\section{b) U238 alpha decay}

Alpha particles with $4.2 \mathrm{MeV}$ move with $2 \times 10^{7}$ $\mathrm{m} / \mathrm{s}$. We will use the eq. (4) with the aim to discover what was the interaction between the particle alpha and the potential $V(r)$ in the distance $d=3$ $\times 10^{-14} \mathrm{~m}$, because once at that point, the particle should exit U238 with the potential energy in that point.

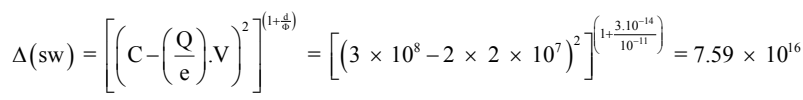

c) Comparison of the potentials $V(r)$ in Rutherford experiment and in U238 alpha decay

From eq. (8) and (9), the ratio between the two $\Delta(\mathrm{sw})$ factors is:

$$
\frac{\Delta(\mathrm{sw})_{\mathrm{RUT}}}{\Delta(\mathrm{sw})_{\mathrm{U} 238}}=\frac{1.44 \times 10^{17}}{7.59 \times 10^{16}}=1.902
$$

As the potential energy $E_{R U T}$ in the distance $d=$ $3 \times 10^{-14} \mathrm{~m}$ is $8.8 \mathrm{MeV}$, then the potential energy $\mathrm{E}(\mathrm{sw})_{\mathrm{U} 238}$ in the alpha decay is:

$$
\mathrm{E}_{\mathrm{U} 238}=\frac{8.8}{1.902}=4.63 \mathrm{MeV}
$$




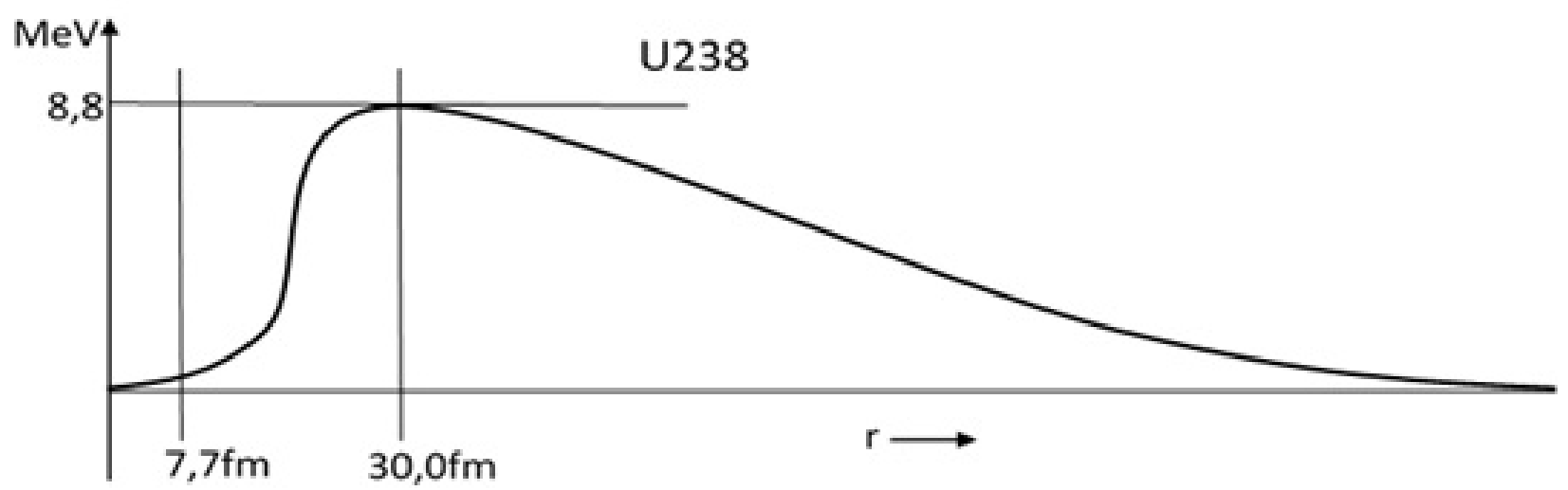

Figure 3: Repulsion between U238 and alpha particle emitted by Po212, in Rutherford experiment, according to the New Coulomb Law.

very close to 4,2 MeV due to the kinetic energy of the alpha particle leaving the U238, measured by experiments. As the alpha particle is accelerated by the potential energy, possibly the difference between 4.63 and $4.2 \mathrm{MeV}$ is due to the irradiation of energy by the accelerated motion.

\section{d) Resume of what happens in U238 alpha decay}

I. In the U238 decay, the point of maximum Coulomb repulsion between Th234 and He4 occurs at the point whose distance " $d$ " (between the center of the Th234 and the center of the alpha particle) is around to $d=30 \mathrm{fm}$.

II. The radius of the Th234 is around $R=7 \mathrm{fm}$, and the radius of $\mathrm{He} 4$ is around $2 \mathrm{fm}$. Then,

A) Consider that the repulsion is ruled by the traditional inverse-square Coulomb's Law.

B) Consider that the alpha particle was bound via strong nuclear force when it was inside the U238.

C) When the He4 is at the point $d=9 \mathrm{fm}$ the potential repulsion is around $27 \mathrm{MeV}$.

D) As consequence, when $\mathrm{He} 4$ reaches to a point far way the Th234 (where the potential energy is practically null), the alpha particle would have to move with kinetic energy around $27 \mathrm{MeV}$.

E) So, the hypothesis of the existence of the strong nuclear force requires that He4 must move with kinetic energy $27 \mathrm{MeV}$ when the repulsion with Th234 becomes practically null. But the experiments show that its kinetic energy is only $4.2 \mathrm{MeV}$.

III. Consider the hypothesis that strong nuclear force does not exist. Then at the point $d=9 \mathrm{fm}$ the energy repulsion between Th234 and $\mathrm{He} 4$ is not $27 \mathrm{MeV}$. Actually it is lower than $8.8 \mathrm{MeV}$ when $d=9 \mathrm{fm}$, because it grows up from a small value in $d=9 \mathrm{fm}$ until to reach the maximum value $8.8 \mathrm{MeV}$ at the distance $\mathrm{d}=30 \mathrm{fm}$ (see Figure 3).

IV. Between equations 8 and 11 is calculated that, for He4 leaving the Th234, in the distance $d=$ $30 \mathrm{fm}$ the Coulomb potential repulsion is 4.63 $\mathrm{MeV}$ (eq. 11), whereas from the traditional Coulomb's law, used by Rutherford, its value is around $8.8 \mathrm{MeV}$.

a) So, finally we understand why alpha particle does not leave Th234 with kinetic energy 8.8 $\mathrm{MeV}$, because the potential $8.8 \mathrm{MeV}$ at the point $\mathrm{d}=30 \mathrm{fm}$ is regarding to the repulsion on an alpha particle moving toward a nucleus U238, with velocity $V=0.097 \mathrm{c}$, in Rutherford's experiment. Therefore, the value $\mathrm{E}_{\mathrm{RUT}}=8.8 \mathrm{MeV}$ (calculated by Rutherford) is actually larger than the potential $\mathrm{E}_{\mathrm{U} 238}$ for the alpha particle at rest regarding to the U238 at the point $d=30 \mathrm{fm}$ (because Rutherford did not consider that the velocity $0.097 \mathrm{c}$ causes a growth in the potential repulsion between U238 and $\mathrm{He} 4)$. For the alpha particle at rest regarding to the $\mathrm{U} 238$, the potential $\mathrm{E}_{\mathrm{U} 238}$ is proportional to $\mathrm{C}^{2}$, whereas $E_{\mathrm{RUT}}$ is larger because it is proportional to $(c+Q .0 .097 c)^{2}=1.43 c^{2}$, where $Q=2$ is the quantity of charge protons in the alpha particle.

b) As in U238 decay the alpha particle is moving with velocity $V=0.067 \mathrm{c}$ leaving the nucleus, then 
the potential energy $E_{T h 234}$ on the alpha particle is very weaker than $\mathrm{E}_{\mathrm{RUT}}$, because $\mathrm{E}_{\mathrm{Th} 234}$ is proportional to $(c-2 \times 0.067 c)^{2}=0.75 c^{2}$. That's why He4 leaves the Th234 with $4.2 \mathrm{MeV}$.

\section{Fundamental Experimental Discoveries between 2009 and 2013, Proving to be Wrong the Foundations of Current Nuclear Physics}

Many experimental findings in the last decade have brought to light several properties of atomic nuclei that are impossible to be conciliated with the foundations of the current Nuclear Physics. Here we mention some of them.

\section{Beryllium isotopes invalidate the shell model}

The advancement of technology along the last decade has allowed the performance of several experiments measuring the nuclear properties of light atomic nuclei. They are made with the aim of verifying if their nuclear properties fit to what is expected from the principles of the SNP. It was known along decades that all the current nuclear models fail for the calculation of the magnetic moments for exotic nuclei, whose quadrupole moments of several of them also cannot be conciliated with what we expect from the foundations of Nuclear Physics. But, surprisingly, beyond the stubbornness of exotic nuclei in exhibiting magnetic moments impossible to be conciliated with current nuclear models, along the last decade the nuclear theorists are also realizing that exotic nuclei have properties that cannot be conciliated with the current nuclear models. Some of them are very exotic, and then we don't understand why some researchers have a big surprise when new experiments reveal that some isotopes violate the laws expected from the current nuclear models, as occurred in 2012, when the shell-model was invalidated by experiments that measured the size of the charge distribution in the Be12 isotope [9].

So, the question is:

- Shell model is used for the explanation of several nuclear properties.

- As beryllium isotopes invalidate the shell model, this imply that it makes no sense to apply it to those nuclear properties to which the shell model was considered to be successfully applied.

Nevertheless, nuclear theorists use to claim that there is not a unique nuclear model from which all the nuclear properties can be explained. Their argument implies that in the nature there are several different nuclear structures (some of them incompatible with other ones), and those structures work independently, and each of them, in the nature, produce phenomena by laws that cannot be applied to the other structures, because each one of them is responsible for a nuclear behavior which manifests itself for the production of a nuclear property that only that structure can produce. From such philosophical viewpoint, it means that there is a lot of different atomic nuclei in the nature, each one of them working with a special principle which allows to that structure to produce a specific nuclear phenomenon. But speaking seriously, it is obvious that it makes no sense to suppose that there are several different structures producing nuclear phenomena in the nature. Sure that there is only one nuclear structure, working by laws that are applied to all the atomic nuclei, by laws that are always ruled by the same fundamental principles. Then, instead of to suspect that Nuclear Physics was developed from wrong foundations (as a reasonable person must suspect), the nuclear theorists prefer to fool themselves, believing in the assumption that nature is no logic. However, as most comfortable as it may be for the nuclear theorists such philosophical justification, by using a meaningless philosophy, soon or later they will need to abandon such comfortable blind belief that there is nothing wrong with the support pillars of the Nuclear Physics. As said Heisenberg, "What we observe is not nature herself, but nature exposed to our method of questioning". So, what we observe nowadays is not the nuclear structure itself, but the nuclear structure exposed to the method of questioning adopted in the Nuclear Physics.

\section{The mystery of the Be7 electric quadrupole moment}

The electric quadrupole moment for $4 \mathrm{Be} 7$ is not quoted in nuclear tables. It's a great mystery, because:

- Its magnetic moment was measured in1998

- Be7 is the mirror of Li7, whose quadrupole moment was measured as being $Q(\mathrm{Li7})=$ -0.0403 barns, and therefore there is not any reasonable reason to justify why $\mathrm{Q}(\mathrm{Be} 7)$ was never measured. 
- Being Be7 and Li7 mirrors nuclei, the nuclear theorists hope that $\mathrm{Q}(\mathrm{Be} 7)$ must be close to the value for $Q(\mathrm{Li} 7)$.

- And because Be7 and Li7 are mirrors nuclei, they must have the same level of difficulty (or better to say facility) when submitted to experiments for measurement of their quadrupole moments. And as $\mathrm{Q}(\mathrm{Li} 7)$ was already measured, why did not $Q(B e 7)$ ?

- The measurement of the electric quadrupole moment for $4 \mathrm{Be} 7$ is critical for the confirmation of a theory on the solar nucleosynthesis (and therefore of maximum interest for many theorists). Then how to justify that it was never measured, in spite of it is crucial for the confirmation of a very important theory?

- The Be7 life-time is 53 days, and therefore there is not any difficulty regarding the time for the measurement.

- As $\mathrm{Q}(\mathrm{Be} 7)$ is very close to zero, according to the author's Hexagonal Floors model, he suspected that the reason why $\mathrm{Q}(\mathrm{Be} 7)$ is missing in nuclear tables is because, in spite of it was already measured by several experimentalists, however they decided do not publish their results, because they measured $\mathrm{Q}(\mathrm{Be} 7)$ very close to zero, and thereby they were afraid that some mysterious error occurred in the measurement. In another words, they gave up of publishing their results, because they knew that $\mathrm{Q}(\mathrm{Be} 7)$ very close to zero is impossible by considering the foundations of the current Nuclear Physics.

- Along 2013 the author exchanged several emails with experimentalists involved in the measurement, among them Dr. Stone, Editor of the nuclear table published by Clarendon Laboratory. No one among them knew how to explain, with a reasonable justification, the mystery of missing $\mathrm{Q}(\mathrm{Be} 7)$ in nuclear tables.

\section{Strong nuclear force denied by radius $7 \mathrm{fm}$ of halo neutron in Be11}

Gamow's paradox implies that protons and neutrons cannot be bound via strong nuclear force inside the atomic nuclei, as already shown herein. And as the strong force does not play any rule for the stability of the stable atomic nuclei, this means that we don't need to consider it any longer, and we conclude that strong nuclear force does not ex- ist.

Interestingly, such conclusion inferred from an impartial analysis of what really happens in the alpha decay of U238, was confirmed in 2009 by an experiment, because while the range of maximum actuation of the strong force is less than $3 \mathrm{fm}$, the experiment has detected that a halo neutron in Be11 is separated from the rest of the nucleus by a distance of $7 \mathrm{fm} \mathrm{[10].} \mathrm{And} \mathrm{any} \mathrm{theory,} \mathrm{supported}$ by the principles of the Standard Nuclear Physics, proposed to explain the enigma, is unacceptable and unfruitful, because:

- Suppose a theory is proposed (no matter if based on the principles of the SNP, or even if based on new arguments beyond the SM), however by keeping the belief that nucleons are bound via strong nuclear force.

- However the theory is unacceptable, because in $97 \%$ of decays $4 B e 11$ transmutes to $5 B 11$, and therefore the neutron does not leave the nucleus.

- In the $4 B e 11$, the neutron decays into a proton and electron, and the proton turns back to the core (the newborn 5B11). If the strong nuclear force was responsible for the cohesion of nuclei, the proton could never go back to the core, because in a distance of $7 \mathrm{fm}$ the proton cannot interact with the core via strong force, and the classical Coulomb repulsion between the core and the proton would be so strong that the proton would be expelled from the newborn $5 B 11$.

- Therefore $5 B 11$ could never be formed in $97 \%$ of the $4 B \mathrm{~B} 11$ decay.

- Conclusion: Be11 halo neutron demolishes the fundamental pillar of the SNP.

\section{The impossible Z-axis in atomic nuclei}

In spite of according to the foundations of the current Nuclear Theory it is impossible to have a preferential axis for the atomic nuclei, in the page 133 of the author's book Quantum Ring Theory [2] is predicted that atomic nuclei have a preferential Z-axis, along which occurs a symmetrical distribution of protons and neutrons, when $\mathrm{Z}$ and $\mathrm{N}$ are pairs. This prediction of 2006, when the book was published, is corroborated by an experiment of 2013, made by researchers led by Professor Peter Butler from the University of Liverpool, which de- 
tected that $88 \mathrm{R} \alpha 224$ has pear shape [11].

According to the current Nuclear Physics, the nucleus $R \alpha 224$ cannot be pear shaped, because from its foundations all the even-even nuclei must have either a spherical shape (when $Z=N$ ) or an ellipsoidal shape (when $N>1$ ). But according to the discovery of 2013, while Radium 224 is pear-shaped, Radon 220 does not assume the fixed shape of a pear but rather vibrates about this shape, and such finding is in contradiction with what is expected from the foundations that rule the behavior of the nuclear models. Beyond the discovery to be very important for the understanding on the structure of the nucleus, the nuclear theorists think that such puzzle can be also related to questions regarding the fundamental interactions responsible for the working of the structure of the universe. And Dr. Timothy Chupp, a University of Michigan professor of physics, has explained how the theorists are dealing with the puzzle. He thinks that pear shape is special, suggesting that neutrons and protons inside the nucleus take different positions along an internal axis. In another words, the pear shape of Ra224 implies that atomic nuclei have an special z-axis, around which protons and neutrons have a preferential distribution, whose existence is impossible, according to current nuclear models .In resume, in 2013 the discovery that Ra224 is pear shaped confirmed what was predicted in the page 133 of the book QRT, published in 2006, where the preferential distribution of protons and neutrons in atomic nuclei was proposed as:

"The distribution about the z-axis is a nuclear property up to now unknown in Nuclear Physics".

\section{The impossible electric quadrupole moment of the deuteron}

Along the decades since the 1960s, thirty-three models were used for the calculation of the electric quadrupole moment for the deuteron. The last paper on the subject was published in 2005 [12], where the authors write in the page 129 (the References from 5 up to 14 ahead are of that paper):

"So, in our investigation of deuteron tensor polarization we employ thirty-three local potential models of the nucleon-nucleon force. The thirtythree local potentials are denoted by the following notation: GK1, GK3 and GK8 of Glendenning and Kramer [5]; PARIS of Lacomb, et al. [6]; RHC, RSC,
RSCA of Reid [7]; TSB and TSC of de Tourreil and Sprung [8]; HJ of Hamada and Johnston [9]; TRS of de Tourreil, et al. [10]; L1, L2, 2, 4, ..., 6 of Mustafa [11]; r1, r3, ..., r7 of Mustafa, et al. [12]; MHKZ of Mustafa, et al. [13]; and a, b, c, ..., i of Mustafa [14]. These thirty-three potential models have different deuteron properties, such as deuteron quadrupole moment $Q_{D^{\prime}}$ D-state probability $P_{D^{\prime}}$ asymptotic $D$-state amplitude $A_{D}$ and asymptotic ratio $\xi$. The values of these properties are not equal, but have wide range of values in all potential models".

As we realize, as consequence of the adoption of the hypothesis of the strong nuclear force and a wrong model for the deuteron, the nuclear theorists were constrained to create several techniques of calculations, trying to understand the exotic nuclear properties of the deuteron, which according to the authors of [12] is "the lightest of all composite nuclei and has the unique property of having an abnormally low binding energy per nucleon, with no excited bound states. The binding energy $E_{B}$ of the deuteron is $2.22 \mathrm{MeV}$, that is $1.11 \mathrm{MeV} /$ nucleon, which is roughly a factor of eight lower than what is typical for stablenuclei (between $7\{9 \mathrm{MeV}$ ) nucleon)".

The reason why the deuteron is a very exotic nucleon is easy to understand, because there is no repulsion between a proton and a neutron, and as they are attracted by the strong nuclear force (as suppose the nuclear theorists), then the deuteron would ought be the nucleon with the highest binding energy existing in the nature. As it is not, the theorists had no other choice than to try all the sort of assumptions, and all they have failed, because it is impossible to reproduce the properties, of a structure existing in the nature, if one tries to get it by using a model whose structure is different. There is no way to get it, because the math cannot supply equal properties for different structures, unless a dirty math is used. But even by using a dirty math, probably it's impossible to get the same properties for a theoretical structure, where the proton and the neutron are bound by the strong nuclear force, and a physical structure existing in the nature, where the proton and the neutron are bound by other sort of force.

The electric quadrupole moment for the deuteron is calculated in [13], from the graphic on charge 
density of nuclei at the Figure 4 of that paper, together with the calculus between eq. (1) and (2) of that paper, by considering a structure formed by a proton and a neutron with structure $(u, d, u-e)$, where " $u$ " and " $d$ " are the up and down quarks, and " $\mathrm{e}$ " is the electron.

\section{The impossible cold fusion}

Nuclear theorists hate to read about such subject. But in 2019 the nuclear cold-fusion reactor eCat is already in the market, in spite of its work is impossible from the foundations of the current Nuclear Physics. So, as harder as can be for the nuclear theorists to recognize that cold fusion is really possible, and therefore the foundations of Nuclear Physics cannot be right, in the upcoming years they will be obliged to face the reality, because year after year will increase the quantity of customers buying the cold-fusion reactor, since it is impossible to prevent the companies to buy a reactor capable to supply heat at prices very lower than any other source can supply. Companies that need heat, as pulp and paper manufacturers, will be obliged to use cold fusion, otherwise they will go bankrupt, because they will not be able to sustain the competitive prices of manufacturers who adhere to the use of the cold-fusion reactor.

Cold fusion was definitively confirmed in 2014 by experiments made in the universities of Bologna and Uppsala, and the Royal Institute of Technology, Stockholm, Sweden. The results were published by a report known as "Observation of abundant heat production from a reactor device and of isotopic changes in the fuel".

\section{New Nuclear Model with Hexagonal Floors}

As shown in three papers published by SciFed Journal of Nuclear Science, from the new nuclear model proposed by the author are calculated with good accuracy the magnetic moments for exotic nuclei with number of protons $Z$ between 3 and 30 .

The basic foundations for the new nuclear model were proposed in the author's book QRT. After 2006, when the book was published, between 2009 and 2012 the author wrote some papers, and submitted them to some mainstream journals of Physics, but as all they were rejected, he felt himself unmotivated to continue improving his theory. But in 2016, as many of the predictions of the book were confirmed between 2009 and 2015, he decided to take again his research. Between 2016 and 2017 he improved his nuclear model, with the introduction of some nuclear properties which he discovered along the two years of research. The three papers where are calculated magnetic moments for exotic nuclei, and one paper where it is demonstrated the viability of the new Coulomb's Law in distances shorter than Bohr's radius, published by SciFed, are:

\section{1. "Calculation of Magnetic Moments of Light Nuclei with Number of Protons between $Z=3$ and $Z=30$ " [3].}

Subject: It is proposed the structure of the nucleus with a central $2 \mathrm{He} 4$, and the laws that rule the behavior of the nucleons inside the atomic nuclei. Magnetic moments for several exotic nuclei are calculated successfully, among which many of them have never been calculated with satisfactory accuracy from any current nuclear model.

\section{2. "Testing the Equations of the New Nuclear Model of Hexagonal Floors" [4].}

Subject: As the title indicates, the new nuclear model is submitted to the hardest test to which never any current nuclear model survived: to calculate the magnetic moments for exotic nuclei, while also is shown that, qualitatively, the electric quadrupole moments also fit to the experimental data. In particular, the orbit radius $R(d)=0.417 \mathrm{fm}$ (radius of deuteron orbit around the central $2 \mathrm{He} 4$, in 3Li6) is calculated with no any arbitrary assumption (eq. 163, page 30). The procedure of calculation involved at the same time the calculation of the magnetic moment $\mu(\mathrm{Li} 6)=+0.8271 \mu \mathrm{N}$, (eq. 174), in a feedback loop where the conversion of $\mathrm{K}_{\mathrm{TH}}(\mathrm{Li} 6)$ to $\mathrm{K}_{\mathrm{EMP}}(\mathrm{O} 15)$ led to the result $\mathrm{R}(\mathrm{d})=0.417$. The experimental value is $+0.822 \mu \mathrm{N}$. As there was no any arbitrary assumption for the calculation of the orbit radius $\mathrm{R}(\mathrm{d})=0.417$, as consequence the value $\mu(\mathrm{Li} 6)=+0.8271$ was calculated with no any arbitrary assumption, with an accuracy of $\Delta=$ $0.0051 \mu \mathrm{N}$.

\section{3. "Mathematical Confirmation for the Nuclear Properties $K(\mathrm{O})=K(\mathrm{Ca})=1 / 2 . K(\mathrm{Si})=1 / 2 . K(\mathrm{Fe})$ " [5].}

Subject: Beyond the successful calculation of magnetic moments for iron isotopes, using an equation obtained from the behavior of the excited silicon-28, in the paper is also addressed the subject regarding the Gamow's unacceptable quantum tunneling theory. In the end of the paper it is also 
exhibited a successful calculation from which is obtained the same tax of nuclear fusions in the stars, without the need to consider the quantum tunneling proposed by Gamow.

\section{4. "Demonstration of the New Coulomb's Law F = $K Q q / d^{X}, X<2$, for Distances $d$ between $10^{-15} \mathrm{~m}$ and $10^{-12} \mathrm{~m}^{\prime \prime}[8]$.}

Subject: Here it's shown how the strings of the electric fields, composed by elementary fermions of the quantum vacuum, interact one each other, so that violate the inverse-square law in the short distance within the atomic nuclei, with repercussions in the field of Particle Physics.

\section{Structure of the author's new nuclear model}

It is composed by:

a) A central alpha particle

b) A flux of gravitons produced by the spins of the protons and neutrons that compose the central $2 \mathrm{He} 4$. This flux is named $\mathrm{n}(\mathrm{o})$-flux.

c) The nucleus has two sides, "Ana" and "Douglas", and they compose a magnet, being Douglas the North pole, and Ana the South pole.

\section{Resume of the nuclear properties of the Hex- agonal Floors model}

\section{A) First property. On the rotation of even $Z=N$ nuclei at the ground state}

The first important nuclear property of this new nuclear model appears as consequence of the two poles of the magnet. The even $\mathrm{Z}=\mathrm{N}$ nuclei rotate at the ground state, without inducing additional magnetic moment due to the rotation of the electric charges of the protons. Such property does not occur in any current nuclear model, because they are not magnets with two poles, and therefore, if the even $\mathrm{Z}=\mathrm{N}$ nuclei would rotate in the ground state, then the rotation of the protons charges would induce a magnetic moment, and by consequence they could not have null magnetic moments. The null magnetic moment for even $\mathrm{Z}=\mathrm{N}$ nuclei was confirmed by experiments, and that's why any current nuclear model cannot rotate at the ground state. Let us see the consequence of the impossibility for the even $\mathrm{Z}=\mathrm{N}$ nuclei to rotate at the ground state, according to the foundations of the current Nuclear Physics, face to an experiment published by Nature in 2012 [14]. In 2012 the nuclear theorists faced a terrible discovery about the even $\mathrm{Z}=\mathrm{N}$ nuclei at the ground state, demolishing a dogma of 80 years. The experiments detected that even $\mathrm{Z}=\mathrm{N}$ nuclei have ellipsoidal shape, instead of spherical as expected from the foundations of the Standard Nuclear Physics. But nuclei with $\mathrm{Z}=\mathrm{N}$ at the ground state has null quadrupole moment. And as nuclei with ellipsoidal shape cannot have null electric quadrupole moment, the author of this paper sent an email to the journal Nature, asking how that puzzle was solved. The nuclear theorist Martin Freer sent the response ahead. 'The nucleus is intrinsically deformed as shown, but has spin 0. Consequently, there is no preferred orientation in the laboratory frame and thus the experimental quadrupole is an average over all orientations and hence is zero. Experimentally is possible to show that the deformation of the ground state is non zero by breaking the symmetry and rotating the nucleus. Martin'.

His argument makes no sense, because the rotation of the protons charges would induce a magnetic field, and it would be detected by experiments. And this magnetic moment of the even $\mathrm{Z}=\mathrm{N}$ nuclei could be aligned toward the direction of an external magnetic moment, and the measurement of the electric quadrupole moment would have to detect a no null value. As conclusion, the discovery that even $\mathrm{Z}=\mathrm{N}$ nuclei have ellipsoidal shape requires that they rotate at the ground state, in spite of the rotation of the charges of their protons do not induce any magnetic moment, and such nuclear property is possible only by considering the new Hexagonal Floors model.

\section{B) Second property. Sign of intrinsic magnetic moment does not change with change of spin from up to down}

In the Figure 4 the symbol $\mathrm{B}(+)$ means that, due to the rotation of the nucleus, the magnetic moment +1.913 of the neutron at the right (with up-spin) induces a positive magnetic moment. Note: in order to find easily, in the several figures of a paper, what nucleons have $\mathrm{B}(+)$ or $\mathrm{B}(-)$, for each nucleon in the figures was adopted to illustrate it by a black circle, with an white sign "+", or "-", inside it. From the Figure 4 one also realizes two nuclear properties:

a) Due to the interaction between the vector $n(0)$ flux and a neutron. When a neutron with magnetic moment +1.913 changes its spin from up to down (or vice-versa), due to its interaction with the $n(0)$-flux its magnetic moment continues to 
be +1.913 . So, the contribution of each of the two neutrons shown in the figure, for the total magnetic moment of the nucleus, is the same, a positive +1.913 . The same property is applied to protons and deuterons.

b) Different signs for magnetic moments induced by rotation. In the Figure 4 , the vector $\mathrm{n}(0)$-spin is down, and so, due to the rotation of the nucleus, the neutron with up-spin induces $\mathrm{B}(+)$, while the neutron with down-spin contributes with $B(-)$, in spite of both them have positive intrinsic $\mu=+1.913$. It happens with neutrons and also with protons and deuterons. The situation in the

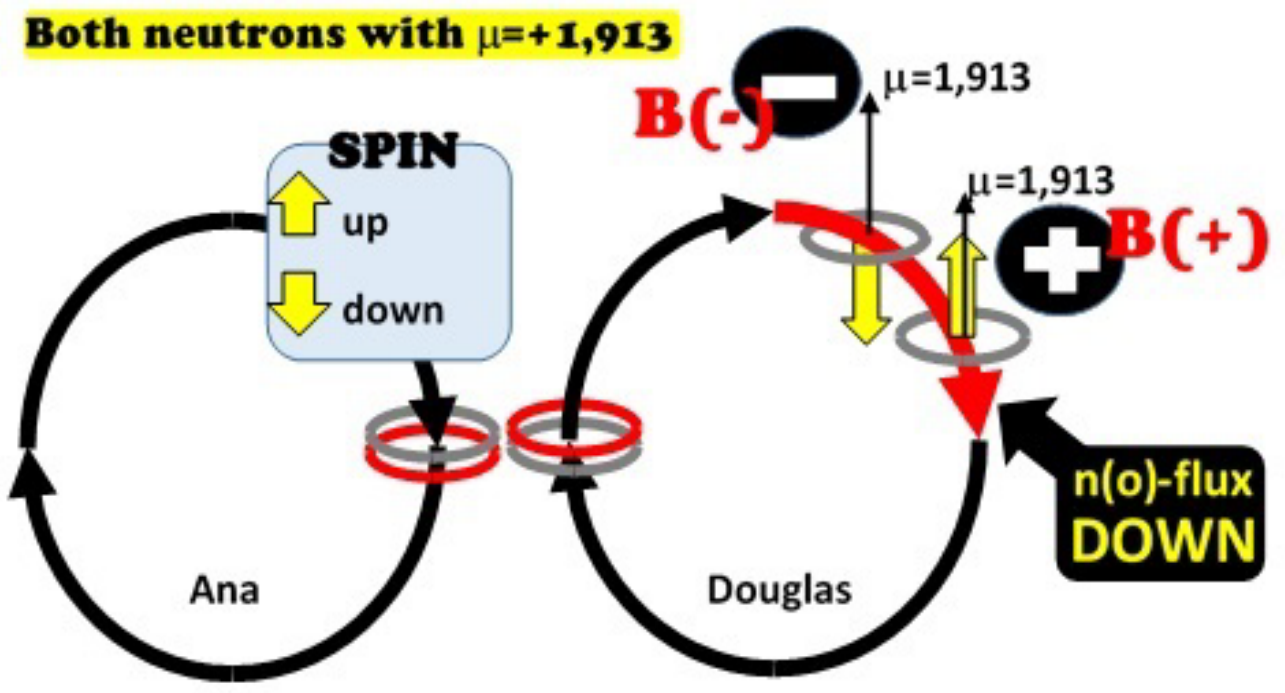

Figure 4: The sign of the intrinsic magnetic moment of a nucleon does not change if its spin is changed from up to down, when the nucleon is crossed by the $n(0)$-flux of the central $2 \mathrm{He} 4$.

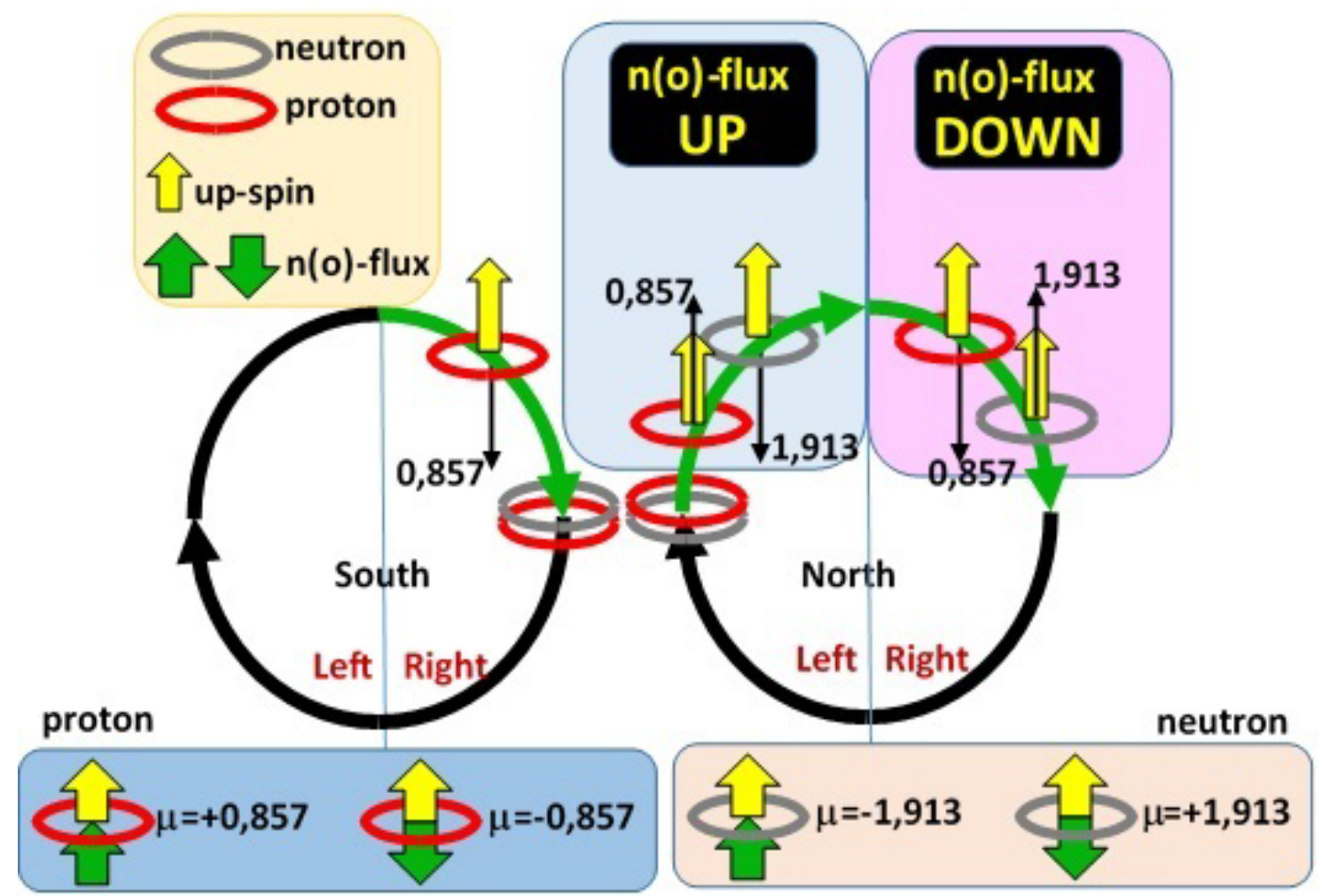

Figure 5: Signs of magnetic moments 0.857 and 1.913 of proton and neutron in a nucleus with positive rotation $R(+)$. 
Figure 4 occurs for positive rotation $\mathrm{R}(+)$ of the nucleus. For the case of a negative rotation $\mathrm{R}(-)$ of the nucleus, the signs of $\mathrm{B}(+)$ and $\mathrm{B}(-)$ in the Figure 4 convert to $B(-)$ and $B(+)$.

C) Third property. On the contribution of the intrinsic magnetic moments of protons, neutrons, and deuterons

The total magnetic moment of a nucleus is due to:

- The sum of the intrinsic magnetic moments of all protons, neutrons, and deuterons

- The total magnetic moment induced by the rotation of those nucleons around the central $2 \mathrm{He} 4$.

- For the calculation of the total magnetic moment of a nucleus, a third important nuclear property is shown in the Figure 5 . The signs of the contribution of $\mu=0.857$ and $\mu=1.913$ of two protons and two neutrons, all them with up-spin in that figure, depends on:

- Regarding magnetic moments induced by rotation, it depends on whether the nucleus has positive rotation $\mathrm{R}(+)$ (counter-clockwise) or negative $\mathrm{R}(-)$.

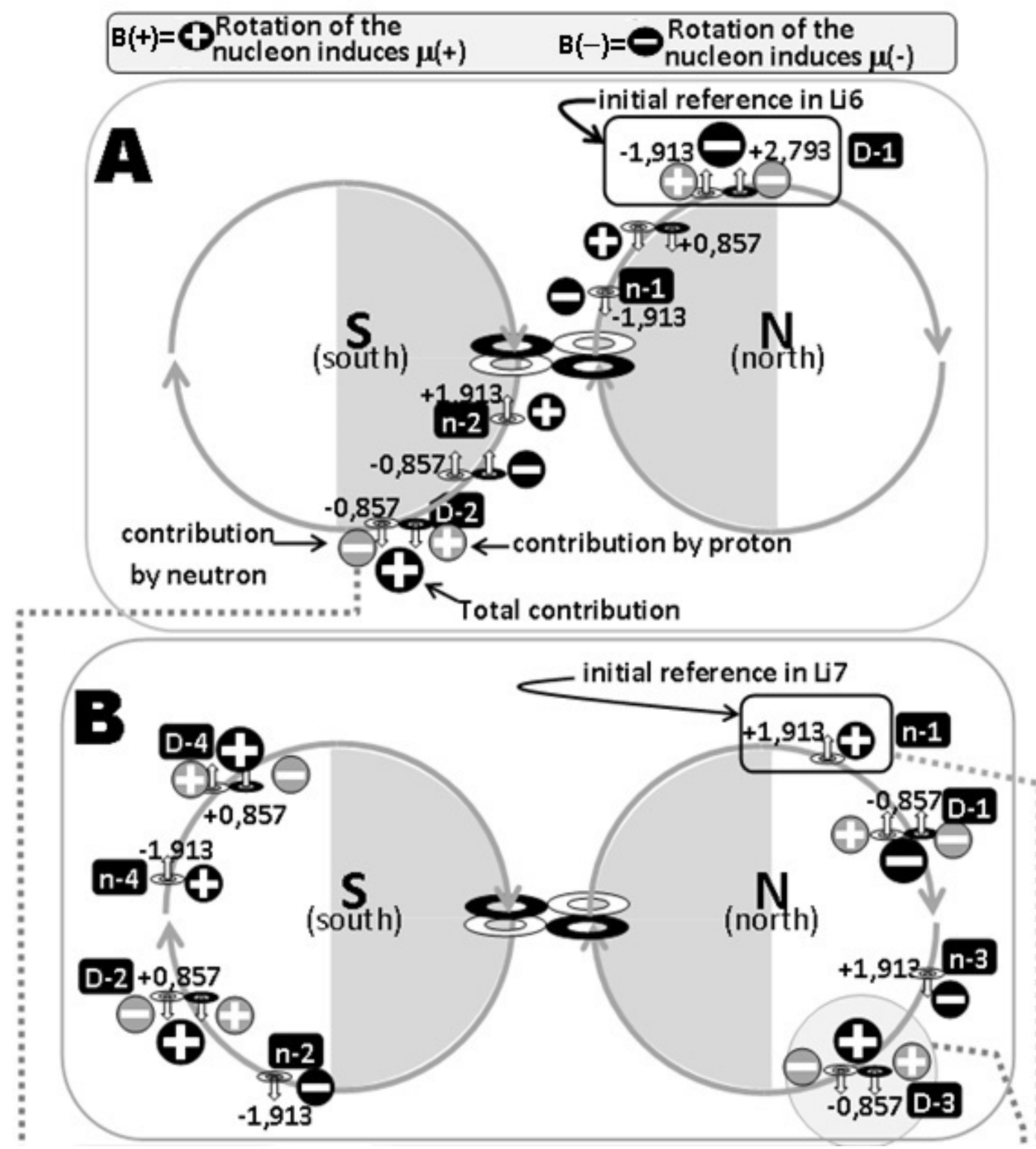

Figure 6: The signs for deuterons and neutrons in several positions. 
- Regarding magnetic moments intrinsic of each nucleon, it depends on whether the nucleon is trespassed by a vector $n(0)$-flux-up or $n(0)$-fluxdown, and whether the nucleon is in the North or South pole of the nucleus.

- Whether a nucleon has $B(+)$, or whether it has $\mathrm{B}(-)$. And $\mathrm{B}(+)$ and $\mathrm{B}(-)$ depends on the position of the nucleon, if in Left or Right side of North pole, or if in the Left or Right side of South pole, and also on the sign of the rotation of the nucleus, whether $\mathrm{R}(+)$ or $\mathrm{R}(-)$.

Figure 5 shows the change of the signs of the intrinsic magnetic moments for protons and neutrons, for positive rotation $\mathrm{R}(+)$, all the nucleons with up-spins. Figure 6 shows the signs for neutrons and deuterons, in several positions, deduced from the magnetic moments of two isotopes, $3 \mathrm{Li} 6$ and 3 Li7.

\section{D) Forth property. The power-rotation of a nucleus, PwR}

Each nucleon contributes with its intrinsic capacity for the power rotation of the nucleus. For the first hexagonal floor, we have:

- The spin of a nucleon contributes for the direction of rotation of the nucleus. In the Douglas side, up-spins of nucleons contribute for positive rotation $\mathrm{R}(+)$ of the nucleus, and down-spins contribute for $\mathrm{R}(-)$. In the Ana side, up-spins contribute for $\mathrm{R}(-)$, and down-spins contribute for $\mathrm{R}(+)$.
- The value of the contribution depends on the intrinsic magnetic moment of the nucleon. Por instance, regarding the intrinsic $P w R(n)$ contribution of a neutron:

- It is proportional of the intrinsic magnetic moment, 1.913. For the proton, it is proportional to 2.793 , and for the deuteron it is proportional to 0.857 .

- The total contribution of several nucleons, for the total $\operatorname{PwR}(X)$ of an isotope $X$, is inversely proportional to the inertia moment of the nucleus, whose value is $I m=A \cdot R^{2}$, where $A$ is the atomic mass of the nucleus, and $R$ is the radius of the nucleus, $R=1.25 \cdot A^{1 / 3}$.

E) Fifth property. Induction-factors $K_{E M P}$ and $K_{T H}$

The rotation of a nucleus $X$ induces an additional magnetic moment $\mu(A d)$, which added to the sum $\Sigma \mu(n, p, d)$ of all intrinsic magnetic moments of neutrons, protons, and deuterons, result in the total magnetic moment $\mu(X)$ of the nucleus. The K-factor which gives the tax of induction of a nucleus is named "induction-factor". There are two sort of $\mathrm{K}$-factors. The empirical induction-factor $\mathrm{K}_{\mathrm{EMP}}$ of a nucleus $X$ is calculated from the experimental value of $\mu(X)$, and it can be used for the calculation of magnetic moments for several isotopes. The theoretical $\mathrm{K}_{\mathrm{TH}}$ is calculated from an equation.

Figure 7 shows the structure of oxygen-15, and ahead is shown how is calculated its empirical induction-factor $\mathrm{K}_{\mathrm{EMP}}(\mathrm{O} 15)$, which is used for calcu-
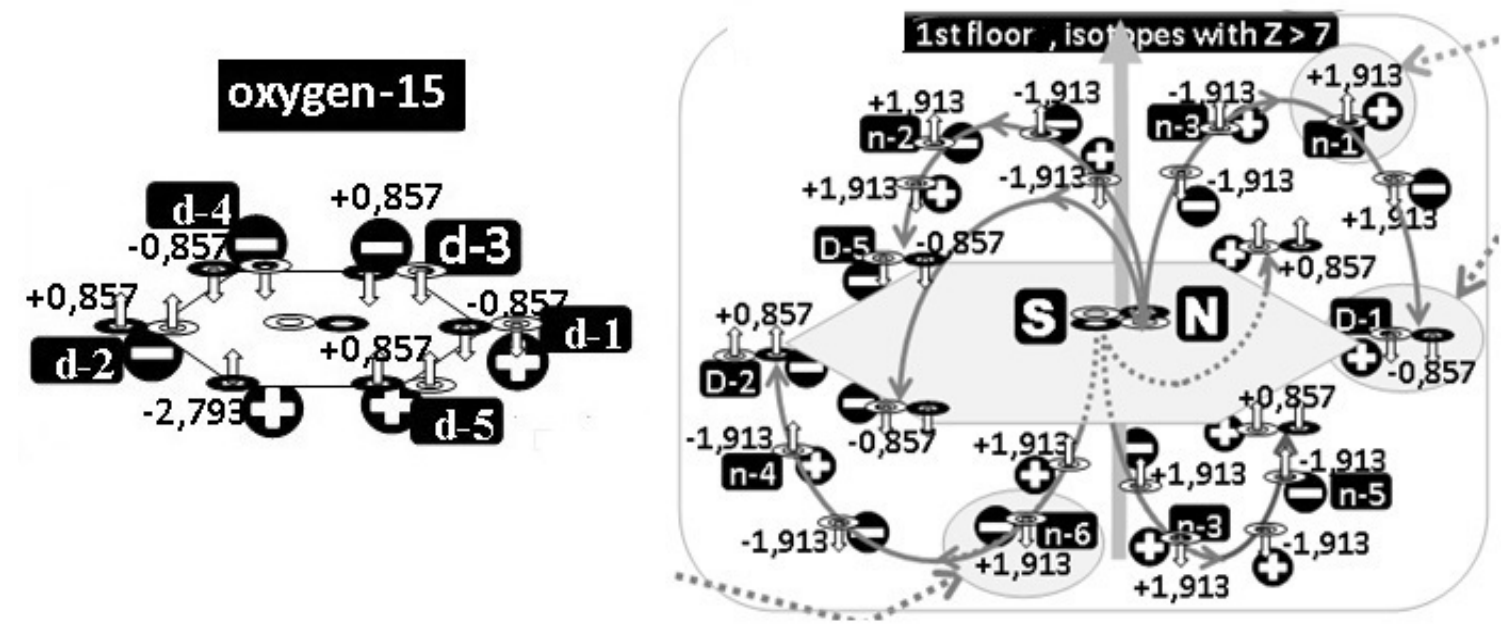

Figure 7: At left: the hexagonal distribution of nucleons in the unique floor of oxygen-15. At right: how the vectors $n(0)$-flux are distributed in the oxygen-16, and the signs of $B(+)$ and $B(-)$ for each deuteron and neutron. They are used as reference in oxygen-15. Note: the $\mathrm{B}(+)$ - factor is represented in the figures by the white sign "+" inside a black disk, and B(-) by the black disk with "-“. 
lation of magnetic moments for isotopes with odd quantity of hexagonal floors, as oxygen, calcium, sodium, vanadium, etc.

In nuclear tables the magnetic moment of oxygen-15 is $\mu=0.719 \mu \mathrm{N}$. From the structure of oxygen-15 in the Figure 7 we see that the deuterons $d-1$ and $d-2$ cancel their intrinsic magnetic moments, as also happens with $d-4$ and $d-5$. Remains two contributions, +0.857 by $d-3$ and -2.793 by the proton. As seen in the figure, $d-2$ and $\mathrm{d}-4$ cancel their respective $\mathrm{B}(-)$ and $\mathrm{B}(+)$, as also occurs with $B(+)$ and $B(-)$ of the deuterons $d-1$ and $d-3$. Then remains $B(+)$ of proton and $B(-)$ of the deuteron $d-5$.

$$
\begin{aligned}
& -2.793+0.857+K .(+2.793-0.857)=0.719 \\
& K=(+2.793-0.857+0.719) /(+2.793-0.857) \\
& K(015)=1.3715
\end{aligned}
$$

\section{Calculation of the power-rotation PwR}

A nucleus rotates due to the contribution of

- The intrinsic $m(n)=1,913, m(p)=2,793$, and $m(d)=0,857$, of all nucleons of the nucleus.

- The spins of all nucleons interacting with the magnetic moments in the north and south poles of Douglas and Ana. The spins are always up or down, because are aligned with the z-axis (the axis rotation of the central $2 \mathrm{He} 4$ ). To be up-spin or down-spin depends on the place where the nucleon is situated, and also depends on the direction of the vector $\mathrm{n}(\mathrm{o})$-flux crossing the nucleon.

Look at the central $2 \mathrm{He} 4$ at Figure 6 , where it's seen that the deuteron in the Douglas side has up-spin with $\mu=+0.857 \mu \mathrm{N}$, and in the Ana side the deuteron has down-spin with $\mu=-0.857 \mu \mathrm{N}$. By convention, was established that up-spin has counter-clockwise rotation. Both the two deuterons of the central $2 \mathrm{He} 4$ produce rotation, and then the central $2 \mathrm{He} 4$ rotates, in the ground state, in counter-clockwise direction (later this conclusion is revised). Therefore:

a) In Douglas side: In any nucleus with $Z>2$, all the nucleons with positive up-spin $\mathrm{s}(+)$ produce rotation $\mathrm{R}(+)$ in counter-clockwise direction, and the nucleons with negative down-spin s(-) produce rotation $\mathrm{R}(-)$ in clockwise direction.

b) In Ana side: Occurs the contrary: In any nucleus with $Z>2$, all nucleons with $s(+)$ produce rotation
$\mathrm{R}(-)$ in clockwise direction, while, and nucleons with $\mathrm{s}(-)$ produce rotation $\mathrm{R}(+)$ in counterclockwise direction.

The PWR is calculated as explained ahead:

1. Protons, deuterons, and neutrons, with $s(+)$ in the Douglas side, cancel respectively the powerrotation of protons, deuterons, and neutrons with $\mathrm{s}(-)$ in the same Douglas side.

2. The same is applied to the Ana side.

3. Therefore, there is need to consider, in Douglas side, only those nucleons whose power-rotation are not cancelled by other nucleons.

4. The same is applied for Ana.

5. A neutron with $s(+)$ in Douglas side and a neutron with $\mathrm{s}(+)$ in Ana side cancel each other their contribution for rotation. The same is applied for protons and deuterons.

6. A neutron with $s(+)$ in Douglas side and a neutron with $s(-)$ in Ana side add their contribution for rotation. The same is applied for protons and deuterons.

7. After eliminating the nucleons which cancel their PwR with other ones, PwR is calculated (as explained, for instance, for the oxygen-15 shown in Figure 7, whose atomic mass is $A=15$ ), as follows:

8. In the Douglas side there is one deuteron of the hex. floor, with $\mu(+)$.

9. In the Ana Side there is a proton with $\mu(-)$.

10. The contributions $\operatorname{PwC}(p), \operatorname{PwC}(d), \operatorname{PwC}(n)$, of each proton, deuteron, and neutron, for the power-rotation of an isotope, are defined as follows:

Proton: $\operatorname{PwC}(p)$ contribution $=2.793 / 0.857^{2}=$ 3.7988

Deuteron: $\mathrm{PwC}(\mathrm{d})$ contribution $=0.857 / 0.857^{2}=$ 1.1663

Neutron: $\operatorname{PwC}(\mathrm{n})$ contribution $=1.913 / 0.857^{2}=$ 2.6021

11. Deuterons $d-3$ and $d-5$ cancel each other their respective $s(-)$ and $s(+)$, while $d-4$ and $d-2$ cancel their $s(-)$ and $s(+)$, and therefore no one of them contributes for PwR(O15).

12. Deuteron $d-1$ in the Douglas side has $s(-)$, and so it contributes for negative rotation. Proton 
in the Ana side has $s(+)$, and therefore contributes for negative rotation too. So, as both the proton and $d-1$ contributes for the same direction of rotation, their total contribution is $3.7988 \times 1.1663$. If they had contributions in contrary directions, their total contribution would be $3.7988 / 1.1663$, in a direction determined by the proton contribution, because it has biggest $\mu(p)=2.793$.

13. For oxygen-15, whose radius is $R=1.25 \times 15^{1 / 3}$ $=3.08277$, the power rotation is:

$\operatorname{PwR}(015)=(1.1663 \times 3.7988) /\left(15 \times 3.08277^{2}\right)=$ 0.03108

where $15 \times 3.08277^{2}$ is the inertia moment of the isotope.

Note: When the author initiated his research for the discovery of the laws that rule his new nuclear model proposed in his book QRT, he did not know yet that the nucleus has two poles North and South. And he wrongly supposed that the structure of the central $2 \mathrm{He} 4$ had to be perfectly symmetric, in order to have two protons with contrary spins, and two neutrons with contrary spins (see the two red arrows in the Figure 8 pointing out the contrary spins of the two protons). In this situation, the central $2 \mathrm{He} 4$ would have rotation (in spite of its PwR(He4) would be very weak, because the orbit radii of the protons and neutrons in the $2 \mathrm{He} 4$ are very short). So, the rotation of the $2 \mathrm{He} 4$ was considered in the calculation of magnetic moments in the first version of paper [3], submitted to the International Journal of Modern Physics, in 2016. But due to the fact that the nucleus has two poles, this changes the situation, and actually the two protons have up-spins, and the two neutrons have downspins. Then he finally understood that the central $2 \mathrm{He} 4$ does not contribute for the rotation of the nuclei.

\section{Calculation of magnetic moments}

From the empirical induction-factor $\mathrm{K}(\mathrm{O} 15)=$ 1.3715 we can calculate the magnetic moment for a nucleus $X$ with hexagonal floor, from the following "conversion":

$\mu(X)=K(015) \cdot[R(X) \cdot P w R(X)] /[R(015) \cdot P w R(015)]$

Several conversions are calculated in [3]. But by considering that the rotation (of the electric charge of one proton in the hexagonal floor of the oxygen-15, which has six proton charges), is responsible for a contribution $1.3715^{1 / 6}$, we reach to the following equation for the calculation of a theoretical induction factor $\mathrm{K}_{\mathrm{TH}}(\mathrm{X})$, for any nucleus " $X$ " with $Z>2$, where the number of protons is $\Phi$ :

$$
\mathrm{K}_{\mathrm{TH}}(\mathrm{X})=1.37145^{\frac{\Phi}{6}} \cdot \frac{\operatorname{PwR}(\mathrm{X}) \cdot \mathrm{R}(\mathrm{X})}{\operatorname{PwR}(015) \cdot \mathrm{R}(015)}
$$

This equation is used for the calculation of magnetic moments for several nuclei, in the paper [4].

Note: The value $\mathrm{K}(\mathrm{O} 15)=1.3715$ was calculated

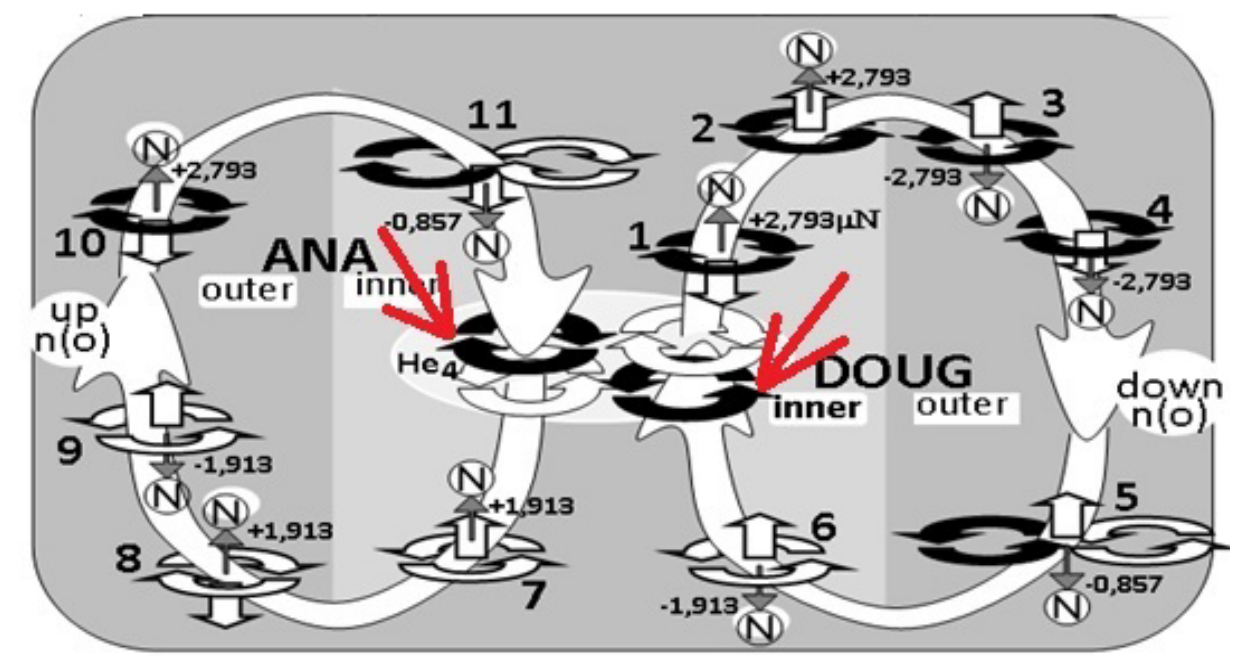

Figure 8: Contrary to what was initially supposed in [4], the protons of the central $2 \mathrm{He} 4$ have no contrary spins(the two red arrows call the attention to their spins in paper [4], where was wrongly supposed that they must be in contrary directions). Later the author changed such initial supposal. So, the central $2 \mathrm{He} 4$ does not contribute for rotation. 

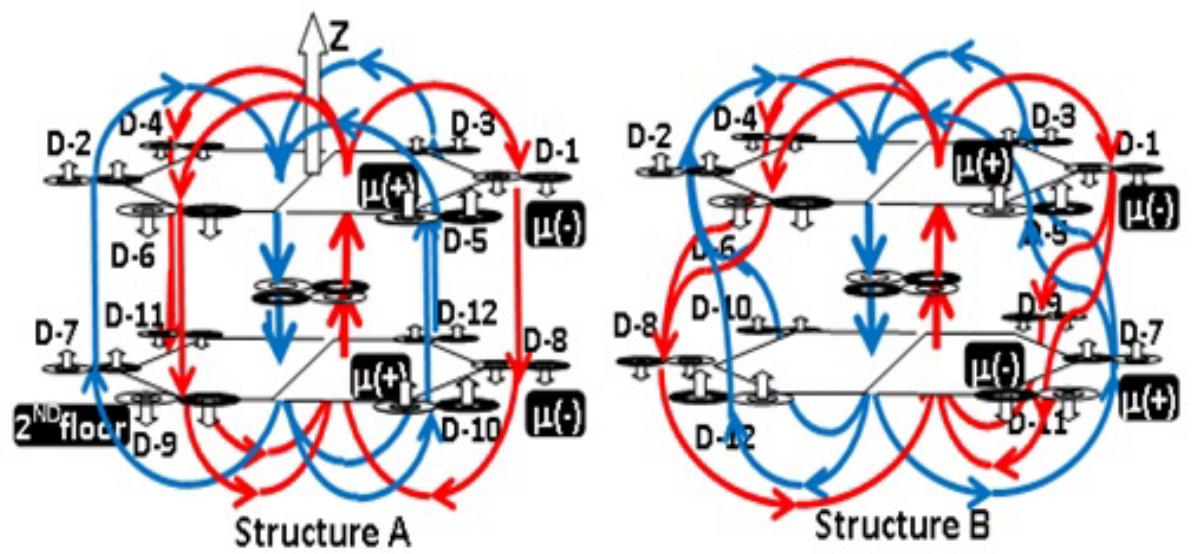

Figure 9: The second floor in silicon isotopes rotates by $180^{\circ}$.

in the first paper [3], published by SciFed, by considering $\mu(015)=0.719 \mu \mathrm{N}$. But the value quoted in nuclear tables is actually $\mu(015)=0.71951 \mu \mathrm{N}$. As magnetic moments are very sensible to any small difference, in his second paper [4] the author calculated again $\mathrm{K}(015)$, with the value $0.71951 \mu \mathrm{N}$, reaching the new value.

$$
\begin{aligned}
& -2.793+0.857+K .(+2.793-0.857)=0.71951 \\
& K=(+2.793-0.8570 .71951) /(+2.793-0.857) \\
& K(015)=1.3717612
\end{aligned}
$$

Mathematical Confirmation for the Nuclear Properties for Oxygen, Calcium, Silicon, and Iron Isotopes

Oxygen isotopes work as one magnet, inducing additional magnetic moments due to the rotation of the nucleus. When the second hexagonal floor is completely formed in silicon isotopes, occurs an interesting anomaly, because the second floor rotates by $180^{\circ}$ regarding the first floor, as shown in the structure $B$ of the Figure 9. If the silicon isotopes had the structure " $A$ " in that figure, their induction-factor would be $\mathrm{K}(\mathrm{Si})=0$, because the first and second hexagonal floors cancel each other their induction-factors. However, as the silicon isotopes rotate by $180^{\circ}$, the induction-factor of silicon isotopes is twice of the oxygen and calcium isotopes, because silicon isotopes work as two magnets, while oxygen and calcium isotopes work as one magnet.

In calcium isotopes two hexagonal floors cancel each other their induction-factor, remaining the contribution of one hexagonal floor only, and therefore oxygen and calcium isotopes have the same induction-factor. Regarding the iron isotopes, they have induction-factor equal to silicon isotopes, because in iron two hexagonal floors cancel each other. This is shown in the Figure 10. In the paper [5], are calculated several magnetic moments for iron isotopes, by using such nuclear property K(Si) $=\mathrm{K}(\mathrm{Fe})$.

\section{Empirical induction-factor K(exSi28) and pow- er-rotation PwR for the second floor}

For isotopes of oxygen (with one hexagonal floor only), from the analysis of the oxygen-15 the author wrongly supposed initially, in the beginning of his research, that:

a) In Douglas side, a nucleon with $\mu(+)$ applies $R(+)$, and with $\mu(-)$ applies $\mathrm{R}(-)$.

b) In Ana side, a nucleon with $\mu(-)$ applies $R(+)$, and with $\mu(+)$ applies $R(-)$.

From the analysis of the behavior of the excited silicon-28, he discovered the true mechanism on how each nucleon contributes for the power-rotation of the nucleus. The rule applied to oxygen isotopes cannot be applied to the silicon isotopes, because their second floor have a rotation by $180^{\circ}$ around the z-axis. In Figure 11, when 13Al28 decays, the deuteron D-1 occupies the last weakly energetically bound state in the newborn silicon-28. Then, when $\mathrm{Si} 28$ at the ground state is excited, the deuteron which change its spin is D-1.

At the Figure 11 occurs the following:

- One of the neutrons of Al28 decays, the newborn proton captures the other neutron, and the newborn deuteron D-8 goes to its place in the first hex. floor. 


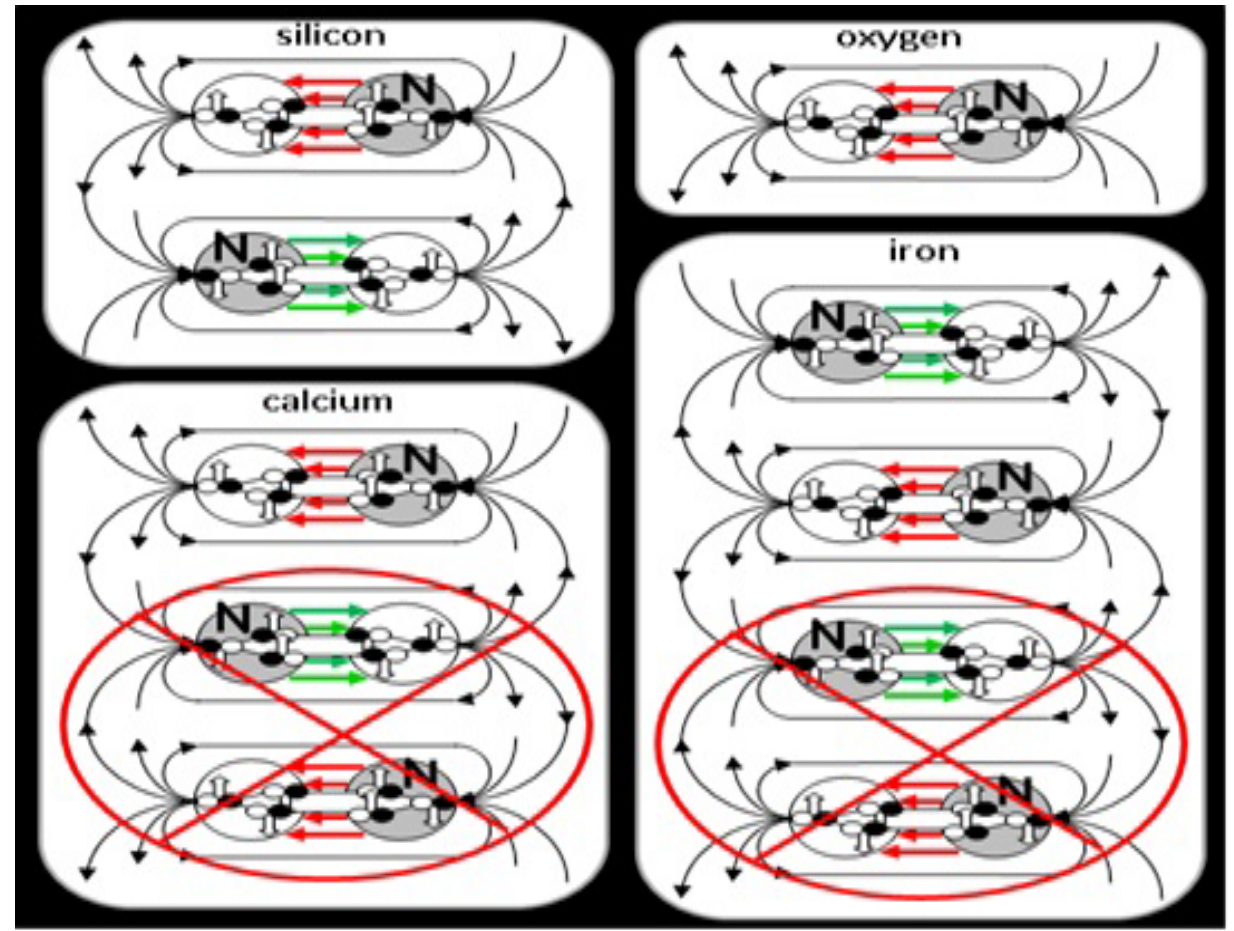

Figure 10: Oxygen and calcium isotopes have one magnet. Silicon and iron isotopes have two magnets.

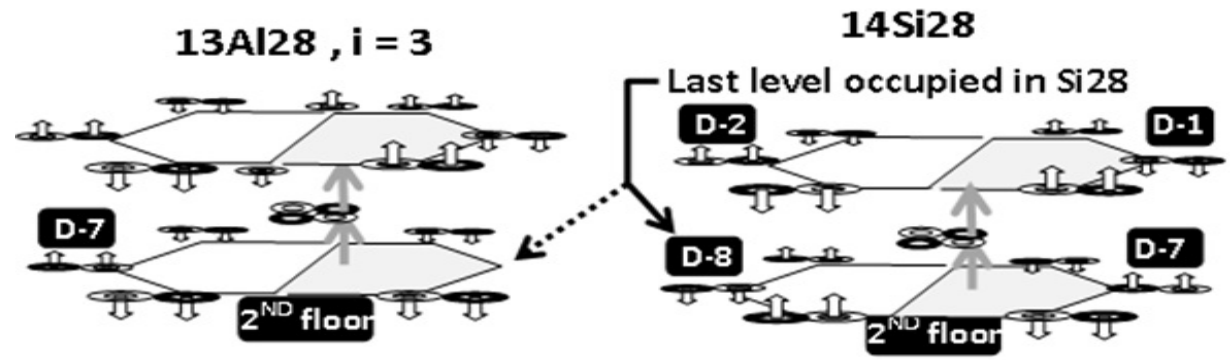

Figure 11: Last level occupied in Si28.

- After being entirely formed the first hexagonal floor, the second floor rotates by $180^{\circ}$, in order that D-7 \& D-8 (in the old Al28) go to take their new places in the newborn Si28.

- The last level in Si28 is occupied by D-8, which is therefore the weakly energetically bound in Si28.

- Ahead is explained what happens when Si28 is excited. At the ground state, Si28 has null nuclear spin and null magnetic moment. Suppose that for silicon isotopes the sign of rotation applied by a nucleon would be consequence of the sign of the intrinsic magnetic moment of that nucleon, as was supposed that occurs for oxygen isotopes. But when Si28 is excited, the deuteron $D-12$ with $\mu=+0.857$ changes its spin from $s(+)$ to $s(-)$, as shown in Figure 12. As known, the sign of the intrinsic magnetic moment of a nucleon, which is crossed by the n(o)-flux, does not change if the nucleon inverts its spin from up to down, or from down to up. As the sign (+) of its intrinsic magnetic moment $\mu=+0.857$ does not change when the spin is inverted, then the excited Si28 would have to continue having null magnetic moment, because PwR(exSi28) would continue being zero, equal to $\mathrm{PwR}(\mathrm{Si} 28)$ at the ground state. Thereby the sign of the intrinsic magnetic moment $\mu=+0.857$ of the deuteron D-12 in Si28 cannot be responsible for the direction of the rotation applied by the PwR(exSi28). There must have other mechanism responsible for the contribution of D-12 for the PwR(exSi28) of the exSi28.

- In the Figure 12 occurs the following: 


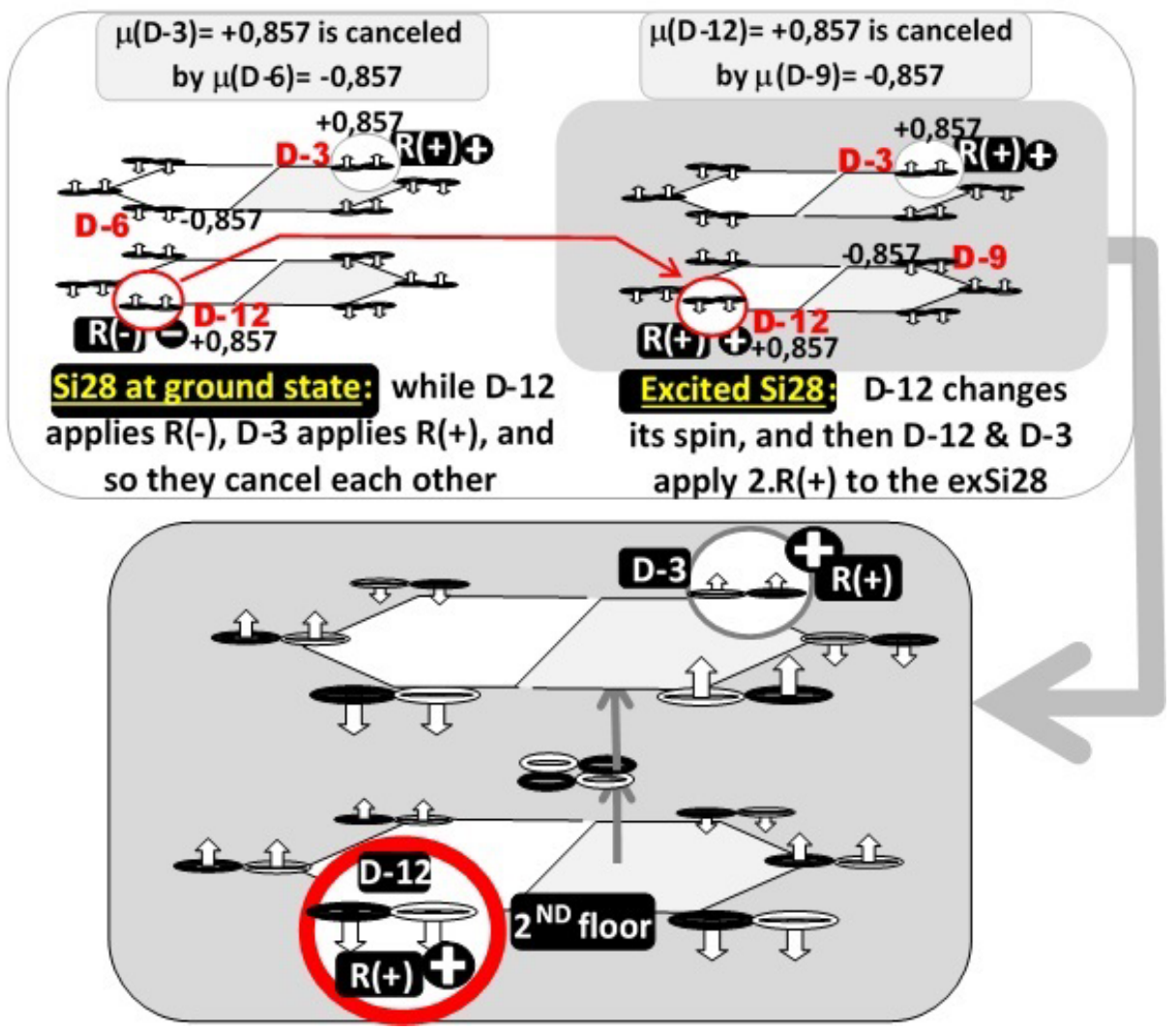

Figure 12: How silicon-28 becomes excited. The deuteron D-12 in Si28 at ground state changes its spin s(+) to $\mathrm{s}(-)$ in excited $\mathrm{Si} 28$.

- Si28 at ground state: All deuterons of the first and second floors cancel their contribution to rotation, and $\mathrm{Si} 28$ rotates does not rotate. While D-3 applies $\mathrm{R}(+), \mathrm{D}-12$ applies $\mathrm{R}(-)$, and so they cancel each other their contribution for PwR(Si28) at the ground state.

- Excited Si28: When D-12 changes its spin, D-3 \& D-12 apply together $2 . R(+)$, and then the excited silicon-28 gyrates with $2 . R(+)$.

So, from Figure 12, we deduce that the powerrotation is driven by the following combination:

a) The intensity of the PwR of an isotope is directly proportional to the intrinsic magnetic moments of protons, deuterons, and neutrons, captured by the $\mathrm{n}(\mathrm{o})$-flux of the central $2 \mathrm{He} 4$.

b) But the direction of the contribution for rotation, applied by a nucleon, depends on the following combinations:

- The direction of the spin: $s(+)$ or $s(-)$.

- Sign of the magnetic pole, $\mathrm{M}(+)$ in Douglas side, or $M(-)$ in Ana side, where the particles is situated, as follows:
- $s(+)$ in $M(+)$ induces $R(+)$

- $s(-)$ in $\mathrm{M}(+)$ induces $\mathrm{R}(-)$

- $s(+)$ in $\mathrm{M}(-)$ induces $\mathrm{R}(-)$

- $s(-)$ in $\mathrm{M}(-)$ induces $\mathrm{R}(+)$

\section{Calculation of empirical $\mathrm{K}_{\mathrm{EMP}}(\mathrm{exSi28})$}

At the top of the Figure 12 are shown the two structures of silicon-28, when it is in the ground state and when it is excited. At the ground state, the deuteron $D-12$ has positive spin $s(+)$, and its PwC(D-12) $=1.1663$ contribution, applied to the rotation of $\mathrm{Si28}$, is negative, $\mathrm{R}(-)$, whereas $\mathrm{D}-3$ contribution $\operatorname{PwC}(D-3)=1.1663$ is positive, $R(+)$, and so they cancel each other their contribution for the PwR(Si28) at the ground state. Therefore, $\mathrm{Si} 28$ has no rotation at the ground state. Note also that at the ground stage D-12 has $\mu(+)$, which is cancelled by $\mu(-)$ of D-9, whereas $D-3$ has $\mu(+)$, which is cancelled by $\mu(-)$ of D-6.

When Si28 is excited, the spin $s(+)$ of $D-12$ is inverted to $s(-)$, and its $\operatorname{PwC}(D-12)=1.1663$ takes the contrary direction, becoming $R(+)$, in the same direction of PwC(D-3). Then the excited Si28 
has rotation $2 . R(+)$. But $\mu(D-12)$ continues being cancelled by $\mu(D-9)$, and $\mu(D-3)$ continues being cancelled by $\mu(D-6)$, so that the total $\Sigma \mu(d)$ due to all intrinsic magnetic moments of deuterons is $\Sigma \mu(d)=0$. But the rotation of D-12 and D-3 induce magnetic moment due to their $\mathrm{B}(+)$. In other words: Excited Si28 has $\mu($ exSi28) $=+1.10 \mu \mathrm{N}$ measured by experiments, induced by the rotation of the nucleus. In $\mathrm{Si} 28$ at the ground state each deuteron of the first floor cancels its $\mathrm{PwC}(\mathrm{d})$ contribution with a deuteron of the second floor, while the intrinsic magnetic moment of each deuteron of the first floor is cancelled by a deuteron of the first floor too. For instance, D-1 of the first floor cancels its $R(-)$ with the $R(+)$ of $D-8$ in the second floor, but D-1 cancels its $\mu(-)$ with $\mu(+)$ of D-2 in the first floor. As $\mu(D-12)=+0.857$ does not change its positive sign when its spin is changed from $s(+)$ to $s(-)$ in excited Si28, then the total contribution of intrinsic spins $\mu=+0.857$ and $\mu=-0.857$ of the whole twelve deuterons continues zero in the excited Si-28. And so one concludes that $\mu($ exSi28) $=+1.10$ is due to only the magnetic moment induced by the rotation of the deuterons D-12 and D-3.

Then now we are able to calculate $\mathrm{K}_{\mathrm{EMP}}$ (exSi28). From Figure 12:

$$
\begin{aligned}
& 2 . \mathrm{K}(\mathrm{exSi} 28) \times 0.857=1.10 \\
& \mathrm{~K}(\mathrm{exSi} 28)=1.10 /(2 \times 0.857) \\
& \mathrm{K}(\mathrm{exSi} 28)=0.6414
\end{aligned}
$$

Its power-rotation is calculated ahead.

$$
\mathrm{R}(\mathrm{Si} 28)=1.25 \times 28^{1 / 3}=3.7957
$$

$$
\operatorname{PwR}(\text { exSi28 })=2 \times 1.1663 /\left(28 \times 3.7957^{2}\right)=
$$
0.005782

\section{Conversion $\mathrm{K}_{\mathrm{EMP}}(015)=>\mathrm{K}_{\mathrm{TH}}(\mathrm{exSi28})$}

As already calculated in eq. (18) that $\mathrm{PwR}(015)$ $=0.03108$, and as $\mathrm{R}(015)=1.25 \times 15^{1 / 3}=3.08276$, then if the isotope oxygen-15 had:

- $A$ radius $\mathrm{R}(\mathrm{O} 15)=\mathrm{R}(\mathrm{Si} 28)=3,795736$

- And a power-rotation PwR(O15) = PwR(exSi28) $=0.00578219$, then the value of its $K_{E M P}(015)$ would be:

${ }_{E M P}(015)=1.371761193 \times \frac{[0.0057822 \times 3.795736]}{0.03108 \times 3.08276}=0.31422782$

The result obtained in eq. (29) shows that, together with eq. (26), lead to the conclusion that, if oxygen-15 was working with the same conditions of exSi28, the ratio $\mathrm{K}(\mathrm{exSi28}) / \mathrm{K}(\mathrm{O} 15)$ would be:

$$
\frac{K(\text { exsi28) }}{K(015)}=\frac{0.6414}{0.31422782}=2.041
$$

This result implies that, if excited silicon-28 would be working in the same conditions under which oxygen-15 works, then its induction-factor $\mathrm{K}_{\mathrm{EMP}}(\mathrm{exSi28})$ would be twice of $\mathrm{K}_{\mathrm{EMP}}(\mathrm{O} 15)$. Therefore, exSi28 has a power for magnetic induction, due to the rotation of the nucleus, twice stronger than oxygen-15, and so silicon isotopes do not follow the eq. (20). For the silicon and iron isotopes, or any nucleus with pair quantity of hexagonal floors, the equation for the theoretical induction-factor is

$$
\mathrm{K}_{\mathrm{TH}}(\mathrm{X})=\left(\frac{\Phi}{6}\right) \cdot 1.37176 \cdot \frac{\operatorname{PwR}(\mathrm{X}) \cdot \mathrm{R}(\mathrm{X})}{\operatorname{PwR}(015) \cdot \mathrm{R}(015)}
$$

where $\Phi=12$, because there are twelve deuterons in each pair formed by two hexagonal floors, and oxygen-15 has only six deuterons in its floor. Equation (31) can also be put either as

$$
\mathrm{K}_{\mathrm{TH}}(\mathrm{X})=2 \cdot \mathrm{K}_{\mathrm{EMP}}(015) \cdot \frac{\operatorname{PwR}(\mathrm{X}) \cdot \mathrm{R}(\mathrm{X})}{\operatorname{PwR}(015) \cdot \mathrm{R}(015)}
$$

Thereby, the theoretical induction-factor $\mathrm{K}_{\mathrm{TH}}(\mathrm{X})$ for calculation of the magnetic moment for a silicon isotope $X$, by using the $K_{E M P}(015)$, is:

$$
\mathrm{K}_{\mathrm{TH}}(\mathrm{X})=2 \times 1,37176 \cdot \frac{\operatorname{PwR}(\mathrm{X}) \cdot \mathrm{R}(\mathrm{X})}{\operatorname{PwR}(015) \cdot \mathrm{R}(015)}
$$

Obviously the calculation can be made by using eq. (34):

$$
\mathrm{K}_{\mathrm{TH}}(\mathrm{X})=\frac{\operatorname{PwR}(\mathrm{X}) \cdot \mathrm{R}(\mathrm{X})}{\mathrm{PwR}(\mathrm{exSi28)} \cdot \mathrm{R}(\mathrm{exSi28})}
$$

From eq. (33), in the third paper [5] published by SciFed, are calculated several magnetic moments for iron isotopes, by using such nuclear property $\mathrm{K}(\mathrm{Si})=\mathrm{K}(\mathrm{Fe})=2 \cdot \mathrm{K}(\mathrm{O} 15)$. As the values obtained are very close to the experimental data, this successful result implies that silicon and iron isotopes really work as two magnets, while oxygen and calcium isotopes work as one magnet.

It's interesting to call attention to some very important results. In paper [3] the magnetic moments of some isotopes are calculated from the eq. (34) of this paper, and in the paper [5] the same isotopes, with the same structure used in paper [3], the calculation is made by using the eq. (32) of this paper, and the results are very close.

$$
\text { Conversion } \mathrm{K}_{\mathrm{EMP}}(\mathrm{O})=>\mathrm{K}_{\mathrm{TH}}(\mathrm{Ca})
$$




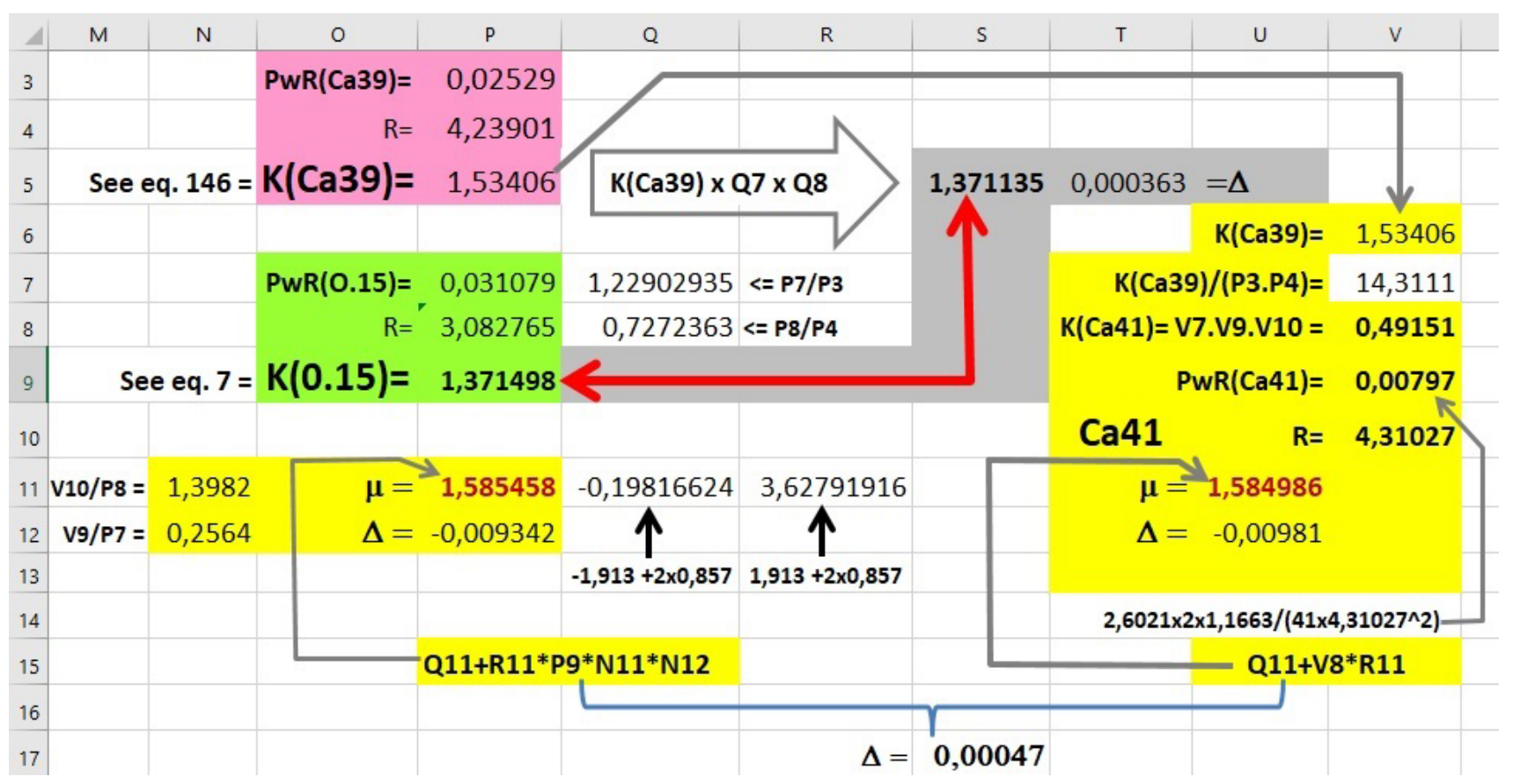

Figure 13: Conversion $\mathrm{K}_{\mathrm{EMP}}(\mathrm{O} 15)=>\mathrm{K}_{\mathrm{TH}}(\mathrm{Ca39})$, calculated by Excel, seen in the Figure 30 of paper [3], by using the eq. (35). And from $\mathrm{K}_{\mathrm{TH}}(\mathrm{Ca39})=1.371135$ is calculated $\left.\mu \mathrm{Ca} 41\right)$, with a difference $\Delta=0.00047 \mu \mathrm{N}$ from the experimental value.

As oxygen and calcium isotopes have only one magnet, then the conversion $\mathrm{K}_{\mathrm{EMP}}(\mathrm{O})$ to $\mathrm{K}_{\mathrm{TH}}(\mathrm{Ca})$ can be obtained by eq. (35).

$$
\frac{K_{T H}(C a)}{K_{E M P}(0)}=\frac{P w R(c a) \cdot R(c a)}{P w R(0) \cdot R(0)}
$$

The most impressive result among several conversions, calculated in [3] and [4], was obtained in [3] with the isotope Ca39, and we actually may consider it as spectacular. As oxygen and calcium isotopes have only one magnet, then it is possible to convert successfully the induction-factor $K(X)$ for an oxygen isotope $X$ to the $K(Y)$ factor for a calcium isotope $Y$. Several conversions $K_{E M P}(O)$ to $K_{T H}(C a)$ are calculated successfully in the paper [3]. But the most striking success occurs in the conversion $\mathrm{K}_{\mathrm{EMP}}(\mathrm{O} 15)$ to $\mathrm{K}_{\mathrm{TH}}(\mathrm{Ca39})$, because both them have an unpaired proton, and because of this reason the two magnets, one of oxygen-15 and the other of calcium-39, have magnetic configurations very, very close. The value $\mathrm{K}_{\mathrm{EMP}}(\mathrm{O} 15)=1.371498$, converted to $\mathrm{K}_{\mathrm{TH}}(\mathrm{Ca39})$, by using the eq. (35) has obtained the value $\mathrm{K}_{\mathrm{TH}}(\mathrm{Ca} 39)=1.371135$, with a difference $\Delta=0.000363$, as seen in the Figure 13 .

\section{New Coulomb's Law for Distances Shorter than Bohr's Radius}

Einstein solved the puzzle of the Michelson-Mor- ley experiment by using the pure mathematics, and he introduced a new way of thinking with his proposal that an empty space, with no any structure, can have physical properties, as its contraction and its inherent capability of producing magnetic fields. The confirmation of his theory led himself and also the physicists to believe that the process of discovery in the field of Physics can be successful by using the pure mathematics. Inspired by that new philosophy, the physicists introduced in the development of the Theoretical Physics the concept that the mysteries of nature can be discovered from a theory developed from a beauty in mathematics, and the first premise to be filled to achieve that beauty would be the symmetry. So, Nuclear and Particle Physics were developed from the concept of symmetry, and as the first fundamental principle required by symmetry is the parity, they were sure that if we look the phenomena by a mirror, we will not see any distinction between the real phenomena and their image in the mirror. But along the decades some phenomena violated the parity principle, as for instance the $\mathrm{Wu}$ experiment. And in 2012 the experiments in the Large Hadron Collider did not detect the particles predicted in the Super symmetry, and nowadays the particle physicists are losing their faith in the beauty of mathematics, as we realize from the book written by the particle 


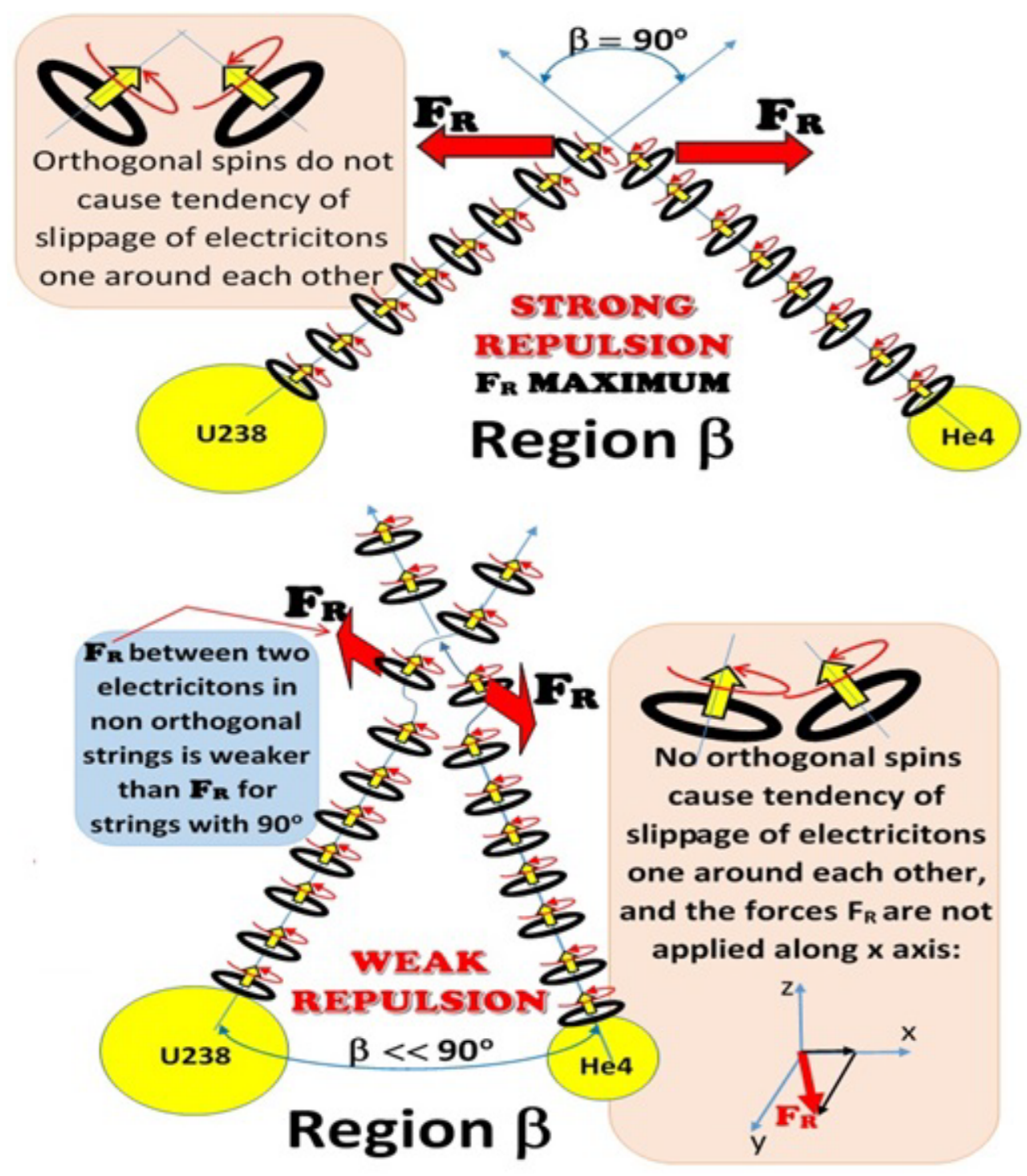

Figure 14: Strong Coulomb interaction occurs between two orthogonal strings.

physicist Sabine Hossenfelder [15], and therefore many of them are sure that there is need to change the philosophy that led the physics astray. Unlike, in spite of the pillars of the Nuclear Physics are being bombarded and demolished, year after year, by more and more experiments, the nuclear theorists continue with their faith in the theory, and there is no way to predict when they finally will realize that their work was developed from wrong initial principles.

The author always believed that phenomena cannot be produced by empty things without structure, as proposed by Einstein. And he always was sure that the structure of the quantum vacuum is responsible for the behavior of light and matter, and this is the reason he felt the need of discovering the physical structure of the electric fields, missing in the current theories. In his first attempt in [7], he wrongly supposed that the interactions, by Coulomb's Law, would be stronger when there is interaction between two parallel strings of fermions of the quantum vacuum (see Figure 1b). And he had supposed that the only challenge there was need to face would be to undertake an effort so that to prove it by mathematics. But later he realized that the inverse-square law cannot be generated from the interaction by two parallel strings, and thereby there was need to introduce some changes in that model of electric field. He did it in the paper [8].

Figure 14 compares two sort of interactions, one by orthogonal strings, and other by strings interacting with angles less than 90 degrees. The stronger interaction by Coulomb's Law actually occurs between two orthogonal strings, and they occur in the 


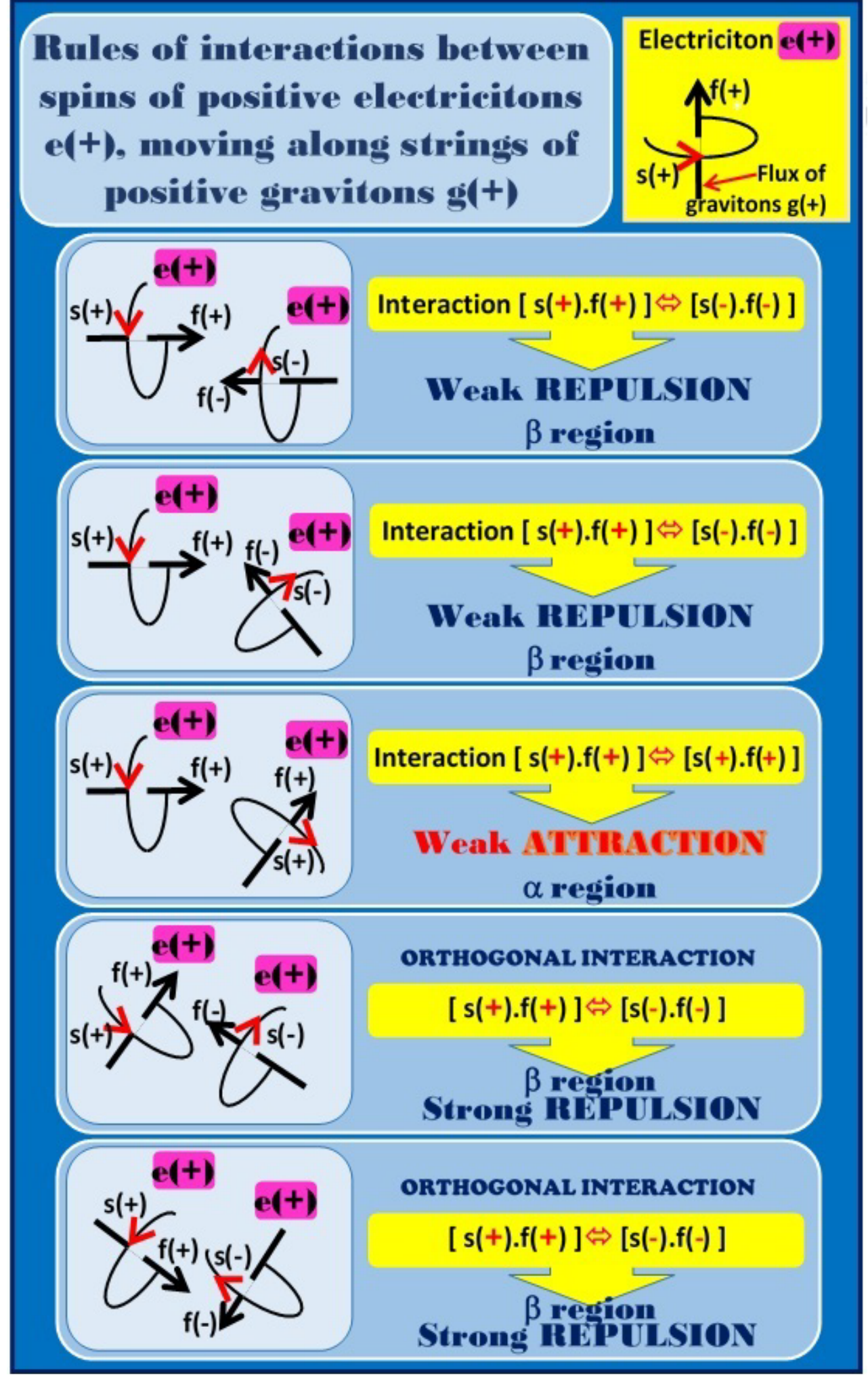

Figure 15: The origin of the weak and strong interactions in the alpha and beta regions, for the interaction of electric fields composed by electriciton se(+).

region $\beta$, also indicated as $\phi(s)$ in the Figure 1 . They are named "Sci" (Sci = strong Coulomb interaction). And weaker Coulomb interactions occur in the regions $\phi(w)$ of the Figure 1 , because in those regions occurs a slippage between two electricitons of two strings, when the angle between the strings is less than 90 degrees. The interaction between electricitons $\mathrm{e}(+)$ of two protons is shown in the Figure 15, and between $\mathrm{e}(+)$ and $\mathrm{e}(-)$ of one proton and one electron is shown in the Figure 16. 


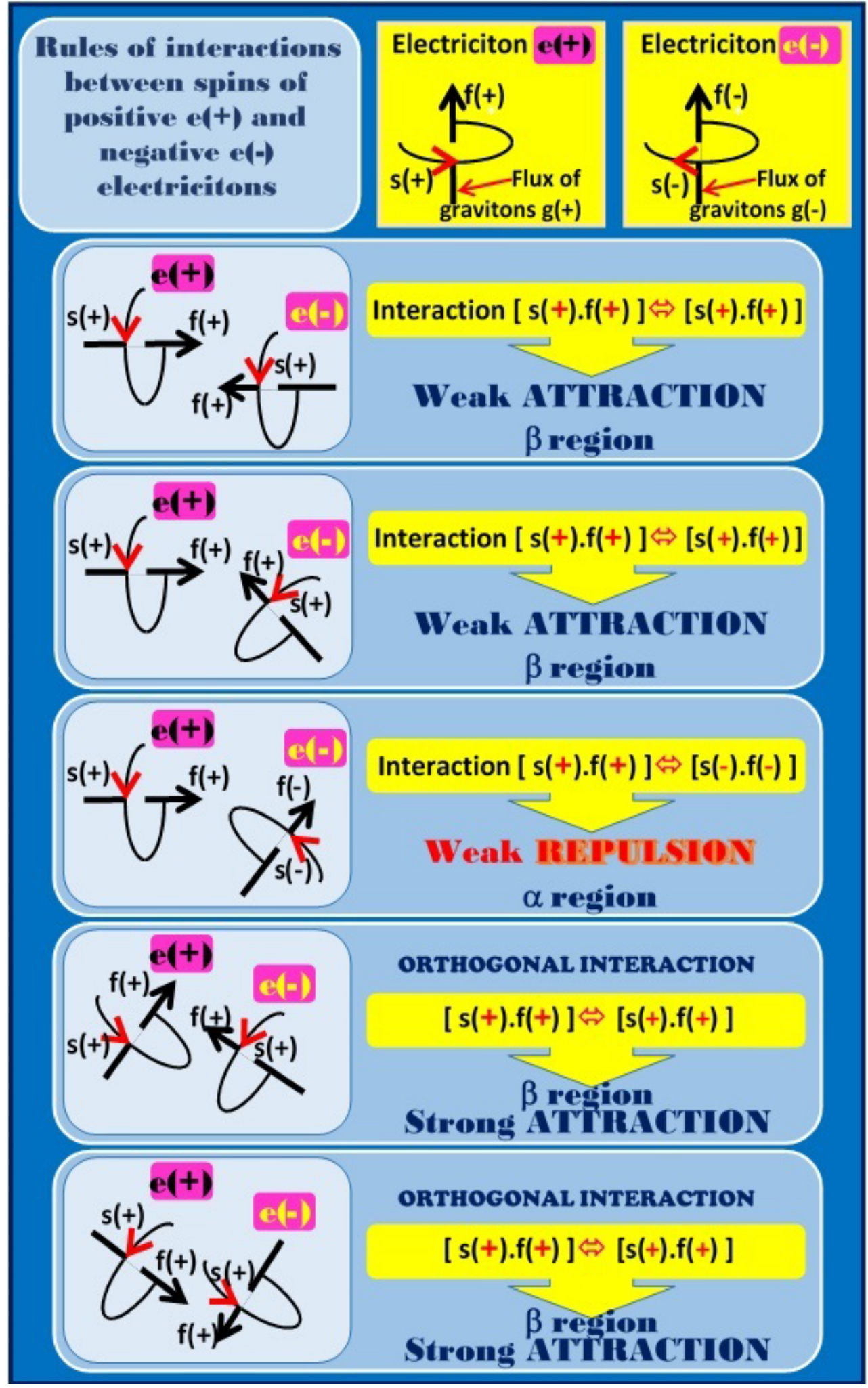

Figure 16: The origin of the weak and strong interactions in the alpha and beta regions, for the interaction between electric fields composed by electriciton se $(+)$ and $\mathrm{e}(-)$, as occurs in the case of the Coulomb attraction between the proton and electron. Note that in the alpha region occurs weak repulsion.

The reason why Coulomb's interactions follow the inverse-square law, for long distances, is shown in the Figure 17. The decrease by the square of distance begins for distances in the range of Bohr's radius.

In order to get a quantitative description on the repulsion force $F_{R}$ between orthogonal strings interacting by Sci, when a particle alpha is at rest re- 


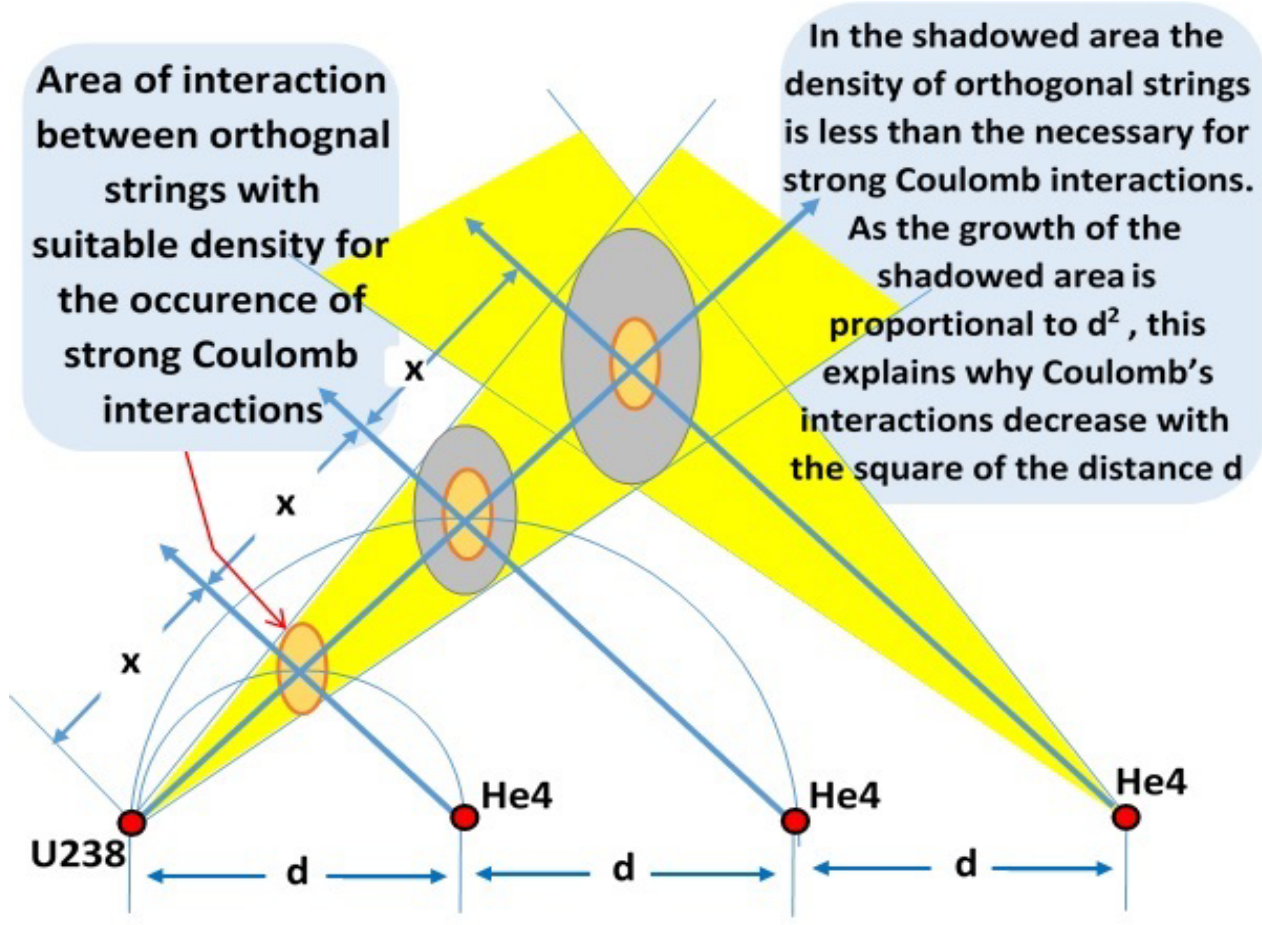

Figure 17: Why Coulomb interactions are inversely proportional to the square of distance, for long distances.

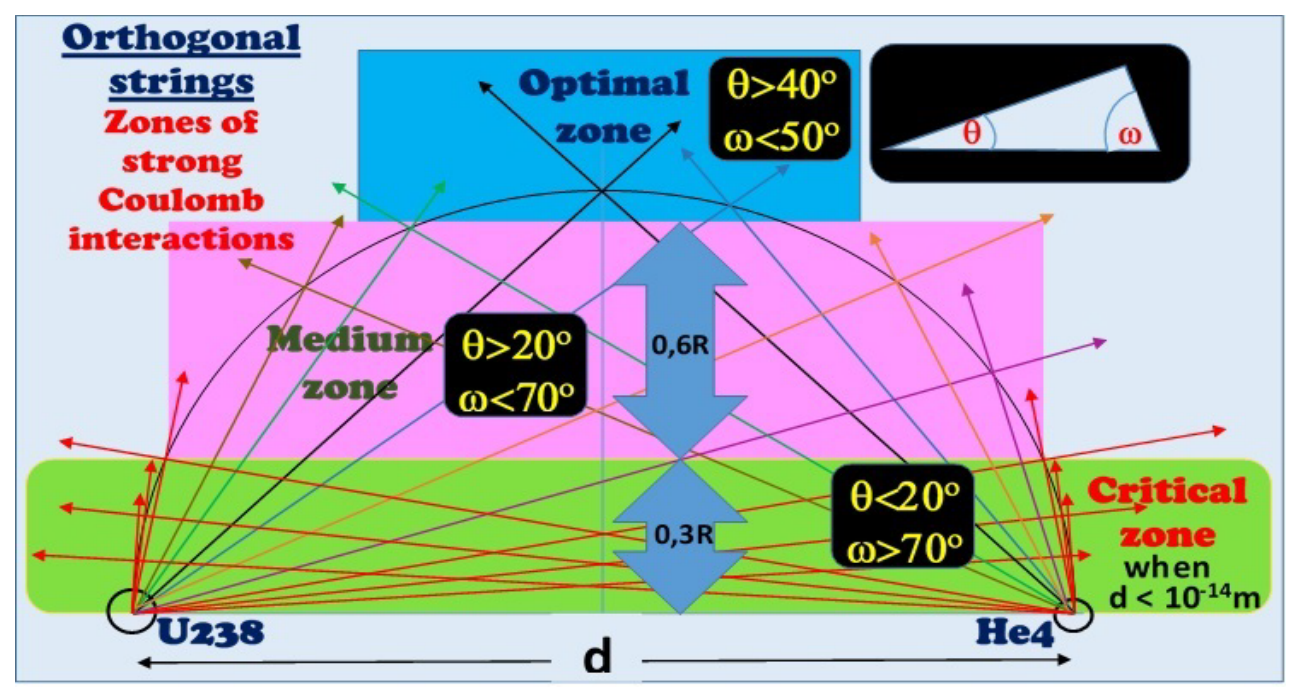

Figure 18: The three zones of interactions by orthogonal strings. The width of the zones are respectively $0.1 \mathrm{R}$, $0.6 R$, and $0.3 R$.

garding a U238 nucleus, the space around the two particles is divided in three zones, named Optimal, Medium, and Critical, as seen in the Figure 18.

The density of the quantity of strings in each of the three zones is calculated in the Figure 19, where is also defined a parameter " $U$ ", with the aim to eliminate an indetermination regarding a question: where is the point, along the distance " $d$ " between the $\mathrm{U} 238$ and the alpha particle, where the interactions by Sci start to decrease drastically?
The three zones of densities $\lambda_{\mathrm{OP}}, \lambda_{\mathrm{MED}}$ and $\lambda_{\mathrm{CR}^{\prime}}$ seen in the Figure 19, are calculated as follows.

a) Critical zone: The strings coming from the U238 are firstly distributed in the yellow cone whose height is $h=2.8+1.4$, seen in the Figure 19. The density $\lambda_{C R}$ is proportional to the area $S_{C R}$ situated in the point with distance $2 \mathrm{~h} / 3=2.8$.

b) Medium zone: The cone trunk is limited by the red line, whose height is $h=5.6+2.8$. The area $S_{M E D^{\prime}}$ used in the calculation of the density $\lambda_{\text {MED' }}$ 


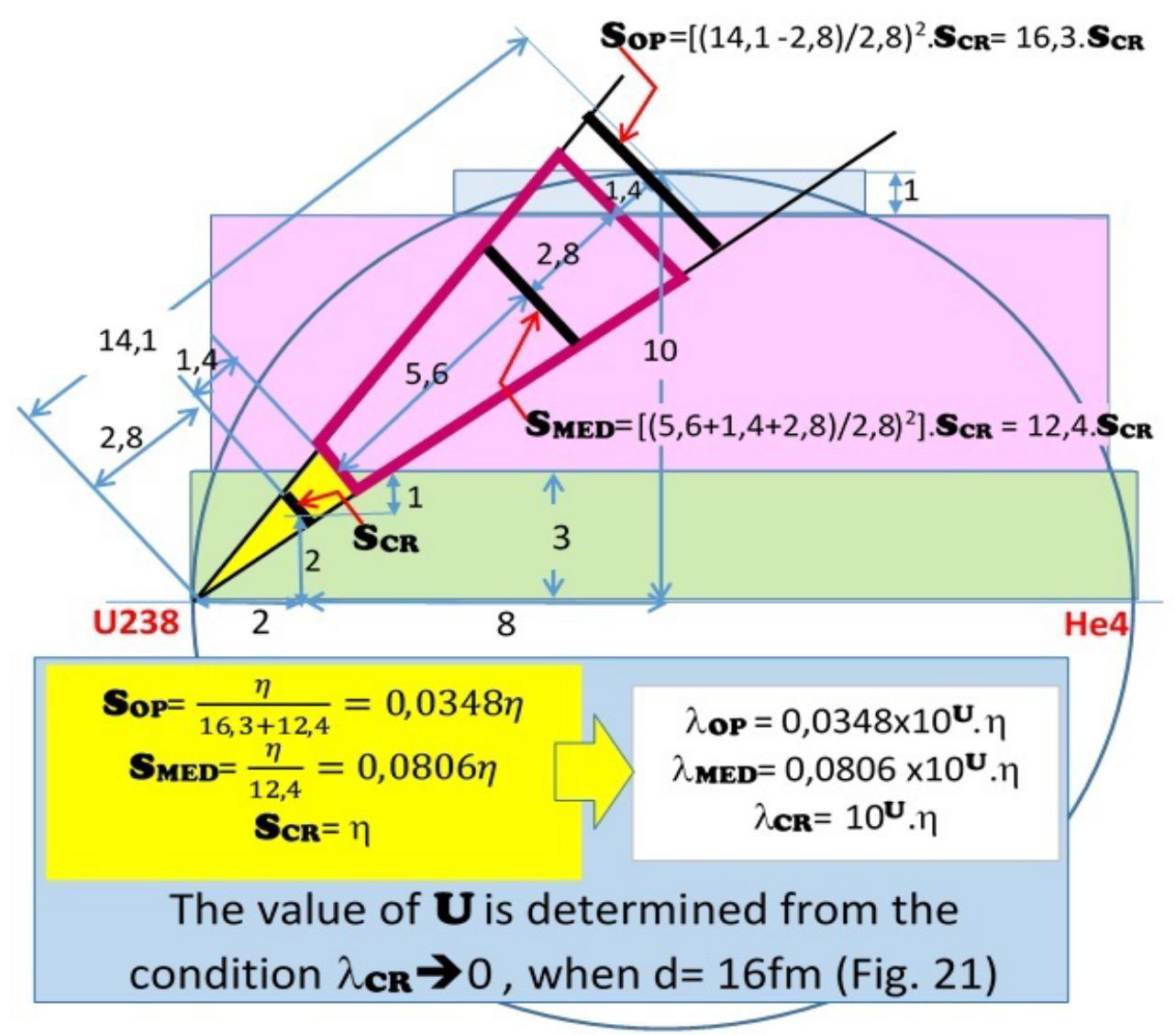

Figure 19: Calculation of the densities of strings in the three zones.

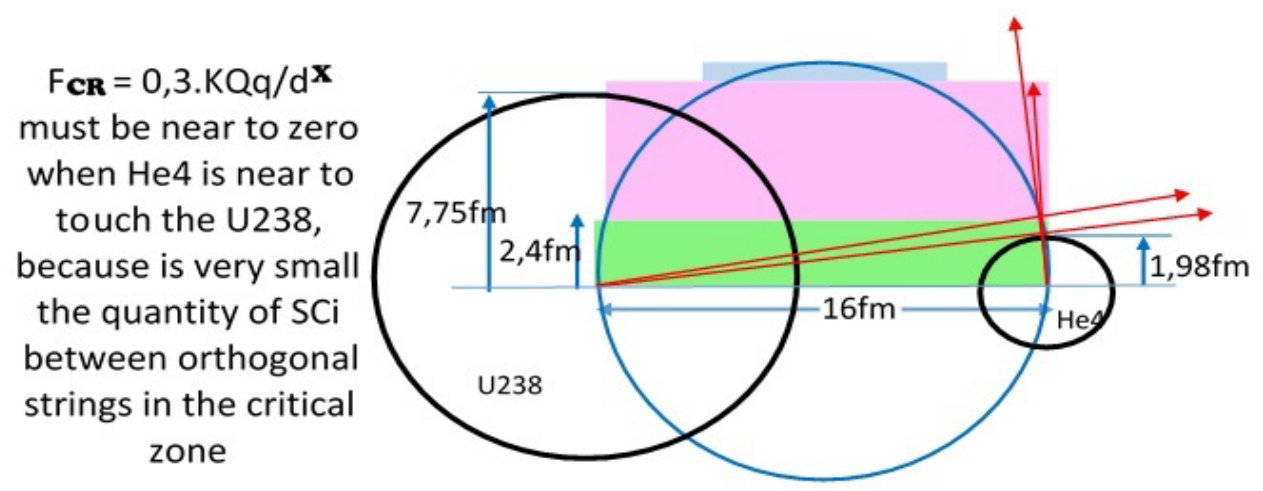

Figure 20: The necessary condition to be satisfied, for the calculation of the parameter $U$, is seen in the figure. When the distance between $\mathrm{U} 238$ and alpha particle is $\mathrm{d}=16 \mathrm{fm}$, the critical force $F_{C R}$ must experience a drastic decrease, because at that point the quantity of interaction between orthogonal strings decreases drastically.

is situated in the point with $h / 3=5.6$.

c) Optimal zone: The area $S_{O P}$ is used for the calculation of the density $\lambda_{\text {op }}$ and it is situated in the distance $d=(1+1)^{1 / 2}=1.4$ from the basis of the trunk cone of the medium zone (as seen, the basis is on the top of the trunk, because it is upside down).

But we have a problem. We don't have any information on how the Coulomb repulsion between
U238 and He4 varies in the range between $1 \mathrm{fm}$ and $30 \mathrm{fm}$. We have only the information given by Rutherford, but even that information is wrong, because he did not consider that Coulomb law varies with the speed $V=3.10^{7} \mathrm{~m} / \mathrm{s}$ of the He4 going toward the U238 (but the relative velocity between the particles must be considered, as shown in [8]). Then, if we begin our graphic plotted by Excel in the point $1 \mathrm{fm}$, the result is different if we begin to plot 
in the point $2 \mathrm{fm}$, or $3 \mathrm{fm}$. And if we use $\Delta=1 \mathrm{fm}$ as the distance between two consecutive points of the graphic, the result is different if we use $\Delta=$ $2 \mathrm{fm}$, or $\Delta=3 \mathrm{fm}$, or $\Delta=4 \mathrm{fm}$.

This problem is solved when we calculate the value of the parameter " $U$ " in $\lambda_{C R}=10^{u} . \eta$. The parameter $\mathrm{U}$ is calculated so that satisfy the condition seen in the Figure 20.

The calculation of the parameter $U$, by Excel, is shown in the Table 1. For the distance $d=$ $1.6 \mathrm{E}-14 \mathrm{fm}$, seen in Figure 20, are calculated in newtons the values of the repulsion force $F_{C R}$ at the "Critical Region", by considering four values for U:

1. $U=0, F_{C R}=49.6282 \mathrm{~N}$.

2. $U=1, F_{C R}=49.1644 \mathrm{~N}$.

3. $\mathrm{U}=2, \mathrm{~F}_{C R}=44.7585 \mathrm{~N}$.

4. $\mathrm{U}=3, \mathrm{~F}_{\mathrm{CR}}=17.5033 \mathrm{~N}$.

5. $U=4, F_{C R}=0.001146 \mathrm{~N}$.
So, from $U=2$ to $U=3$, the tax of change is $44.75 / 17.50=2.56$, but from $U=3$ to $U=4$ the tax change decreases drastically, 17.50/0.0011 = 15909.1. Therefore, occurs a brusque decrease of $F_{C R}$ when $U$ takes the value 4 .

\section{Procedure for the calculation of Coulomb forces in the three zones}

According to the old Coulomb law, when the distance $d$ decreases, the repulsion increases directly proportional to the square of the distance d. So, the phenomenon responsible for the decrease of the intensity of the interaction, when the distance decreases, needs to occur directly proportional to the square of the distance $d$ too. The reason why this occurs is seen in the Figure 21.

The point $d=30 \mathrm{fm}$ is where occurs the maximum SCi interactions, as detected experimentally by Rutherford. With the shrinkage of the distance $d$, the area of interaction diminishes, decreasing

Table 1: Calculation of the parameter $U$.

\begin{tabular}{|c|c|c|c|c|c|c|c|c|c|c|}
\hline 4 & A & B & $\mathrm{C}$ & D & $\mathrm{E}$ & $\mathrm{F}$ & G & $\mathrm{H}$ & 1 & $J$ \\
\hline 15 & & & 1 & 2 & 3 & 4 & 5 & 6 & 7 & 8 \\
\hline 16 & & & $4 E-15$ & $6 \mathrm{E}-15$ & $8 E-15$ & $1 E-14$ & $1,2 \mathrm{E}-14$ & $1,4 \mathrm{E}-14$ & $1,6 \mathrm{E}-14$ & $1,8 \mathrm{E}-14$ \\
\hline 17 & $\lambda_{\mathrm{CR}}=$ & $\mathbf{U}=\mathbf{0} \Rightarrow$ & 0,0625 & 0,027778 & 0,015625 & 0,01 & 0,0069444 & 0,005102 & 0,003906 & 0,003086 \\
\hline 18 & $\mathbf{h}=$ & $6 \mathrm{E}-16$ & $1,2 \mathrm{E}-15$ & $1,8 \mathrm{E}-15$ & $2,4 \mathrm{E}-15$ & $3 E-15$ & $3,6 \mathrm{E}-15$ & $4,2 \mathrm{E}-15$ & $4,8 \mathrm{E}-15$ & $5,4 \mathrm{E}-15$ \\
\hline 19 & $\mathrm{~S}_{\mathrm{CR}}=$ & $7,536 \mathrm{E}-30$ & $3,01 E-29$ & $6,78 \mathrm{E}-29$ & $1,206 \mathrm{E}-28$ & $1,884 \mathrm{E}-28$ & $2,713 E-28$ & $3,693 E-28$ & $4,82 E-28$ & $6,1 \mathrm{E}-28$ \\
\hline 20 & $F_{C R}=K Q q / d^{X}$ & & 728,9082 & 345,411 & 196,9415 & 126,61447 & 88,096635 & 64,786177 & 49,6282 & 39,2248 \\
\hline 21 & $F=K Q q / d^{2}$ & & 794,88 & 353,28 & 198,72 & 127,1808 & 88,32 & 64,888163 & 49,68 & 39,25333 \\
\hline \multicolumn{11}{|l|}{22} \\
\hline 23 & $\lambda_{\mathrm{CR}}=$ & $\mathbf{U}=\mathbf{1} \Rightarrow$ & 0,625 & 0,277778 & 0,15625 & 0,1 & 0,0694444 & 0,0510204 & 0,039063 & 0,030864 \\
\hline 24 & $\mathbf{h}=$ & $6 E-16$ & $1,2 \mathrm{E}-15$ & $1,8 \mathrm{E}-15$ & $2,4 \mathrm{E}-15$ & $3 E-15$ & $3,6 E-15$ & $4,2 E-15$ & $4,8 \mathrm{E}-15$ & $5,4 \mathrm{E}-15$ \\
\hline 25 & $\mathbf{S}_{\mathrm{CR}}=$ & $7,536 \mathrm{E}-30$ & $3,01 E-29$ & $6,78 \mathrm{E}-29$ & $1,206 \mathrm{E}-28$ & $1,884 \mathrm{E}-28$ & $2,713 E-28$ & $3,693 E-28$ & $4,82 E-28$ & $6,1 \mathrm{E}-28$ \\
\hline 26 & $F_{C R}=K Q q / d^{x}$ & & 334,2059 & 282,0269 & 181,63445 & 121,62968 & 86,111601 & 63,87548 & 49,16443 & 38,96897 \\
\hline 27 & $F=K Q q / d^{2}$ & & 794,88 & 353,28 & 198,72 & 127,1808 & 88,32 & 64,888163 & 49,68 & 39,25333 \\
\hline \multicolumn{11}{|l|}{28} \\
\hline 29 & $\lambda_{\mathrm{CR}}=$ & $\mathbf{U}=\mathbf{2} \Rightarrow$ & 6,25 & 2,777778 & 1,5625 & 1 & 0,6944444 & 0,5102041 & 0,390625 & 0,308642 \\
\hline 30 & $\mathbf{h}=$ & $6 \mathrm{E}-16$ & $1,2 \mathrm{E}-15$ & $1,8 \mathrm{E}-15$ & $2,4 \mathrm{E}-15$ & $3 E-15$ & $3,6 \mathrm{E}-15$ & 4,2E-15 & $4,8 \mathrm{E}-15$ & $5,4 \mathrm{E}-15$ \\
\hline 31 & $\mathbf{S}_{\mathrm{CR}}=$ & $7,536 \mathrm{E}-30$ & $3,01 E-29$ & $6,78 E-29$ & $1,206 \mathrm{E}-28$ & $1,884 \mathrm{E}-28$ & $2,713 E-28$ & $3,693 E-28$ & $4,82 E-28$ & $6,1 \mathrm{E}-28$ \\
\hline 32 & $F_{C R}=K Q q / d^{X}$ & & 0,137223 & 37,13935 & 80,873833 & 81,395712 & 68,562257 & 55,443729 & 44,75849 & 36,50068 \\
\hline 33 & $F=K Q q / d^{2}$ & & 794,88 & 353,28 & 198,72 & 127,1808 & 88,32 & 64,888163 & 49,68 & 39,25333 \\
\hline \multicolumn{11}{|l|}{34} \\
\hline 35 & $\lambda_{\mathrm{CR}}=$ & $\mathbf{U}=\mathbf{3} \Rightarrow$ & 62,5 & 27,77778 & 15,625 & 10 & 6,9444444 & 5,1020408 & 3,90625 & 3,08642 \\
\hline 36 & $\mathbf{h}=$ & $6 \mathrm{E}-16$ & $1,2 \mathrm{E}-15$ & $1,8 \mathrm{E}-15$ & $2,4 \mathrm{E}-15$ & $3 E-15$ & $3,6 \mathrm{E}-15$ & $4,2 \mathrm{E}-15$ & $4,8 \mathrm{E}-15$ & $5,4 \mathrm{E}-15$ \\
\hline 37 & $\mathrm{~S}_{\mathrm{CR}}=$ & $7,536 \mathrm{E}-30$ & $3,01 E-29$ & $6,78 \mathrm{E}-29$ & $1,206 \mathrm{E}-28$ & $1,884 \mathrm{E}-28$ & $2,713 E-28$ & $3,693 E-28$ & $4,82 \mathrm{E}-28$ & $6,1 \mathrm{E}-28$ \\
\hline 38 & $F_{C R}=K Q q / d^{X}$ & & $1,87 \mathrm{E}-35$ & $5,82 E-08$ & 0,0247689 & 1,4662948 & 7,0197158 & 13,459687 & 17,50328 & 18,97231 \\
\hline 39 & $F=K Q q / d^{2}$ & & 794,88 & 353,28 & 198,72 & 127,1808 & 88,32 & 64,888163 & 58 & 39,25333 \\
\hline \multicolumn{11}{|l|}{40} \\
\hline 41 & $\lambda_{\mathrm{CR}}=$ & $\mathbf{U}=\mathbf{4} \Rightarrow$ & 625 & 277,7778 & 156,25 & 100 & 69,444444 & 51,020408 & 25 & 30,8642 \\
\hline 42 & $\mathbf{h}=$ & $6 \mathrm{E}-16$ & $1,2 \mathrm{E}-15$ & $1,8 \mathrm{E}-15$ & $2,4 \mathrm{E}-15$ & $3 E-15$ & $3,6 \mathrm{E}-15$ & $4,2 \mathrm{E}-15$ & 15 & $5,4 \mathrm{E}-15$ \\
\hline 43 & $\mathbf{S}_{\mathrm{CR}}=$ & $7,536 \mathrm{E}-30$ & $3,01 \mathrm{E}-29$ & $6,78 \mathrm{E}-29$ & $1,206 \mathrm{E}-28$ & $1,884 \mathrm{E}-28$ & $2,713 E-28$ & $3,693 \mathrm{E}-28$ & $4,2 \geq 28$ & $6,1 \mathrm{E}-28$ \\
\hline 44 & $F_{C R}=K Q q / d^{X}$ & & $\mathbf{0}$ & $5,24 \mathrm{E}-96$ & $1,798 \mathrm{E}-37$ & $5,277 \mathrm{E}-18$ & $8,885 E-10$ & 9,569E-06 & 0,001464 & 0,027309 \\
\hline 45 & $F=K Q q / d^{2}$ & & 794,88 & 353,28 & 198,72 & 127,1808 & 88,32 & 64,888163 & 49,68 & 39,25333 \\
\hline
\end{tabular}


Area $\mathbf{S}_{\mathbf{A}}$ of maximum quantity of SCi orthogonal interaction in the distance $\mathrm{d}=30 \mathrm{fm}$

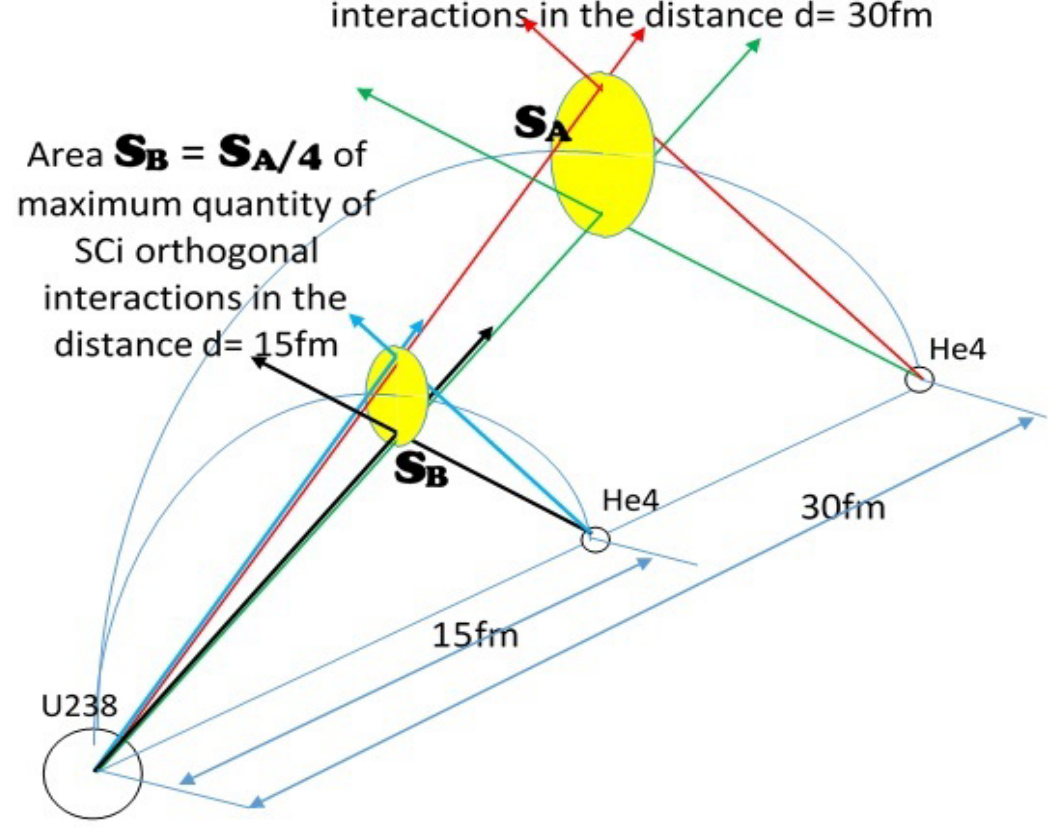

Figure 21: The quantity of SCi orthogonal interactions is inversely proportional to the square of distance $d$. And the tax of maximum quantity of $\mathrm{SCi}$ interactions by unity of area occurs in the distances shorter than $30 \mathrm{fm}$. Because of the saturation of strings in the area $S_{B}$ (caused by the excess of strings in a small area), the strings red and green (which did succeed to produce SCi in the area big area $S_{A}$ ) are not able to produce SCi in the small area $S_{B}$.

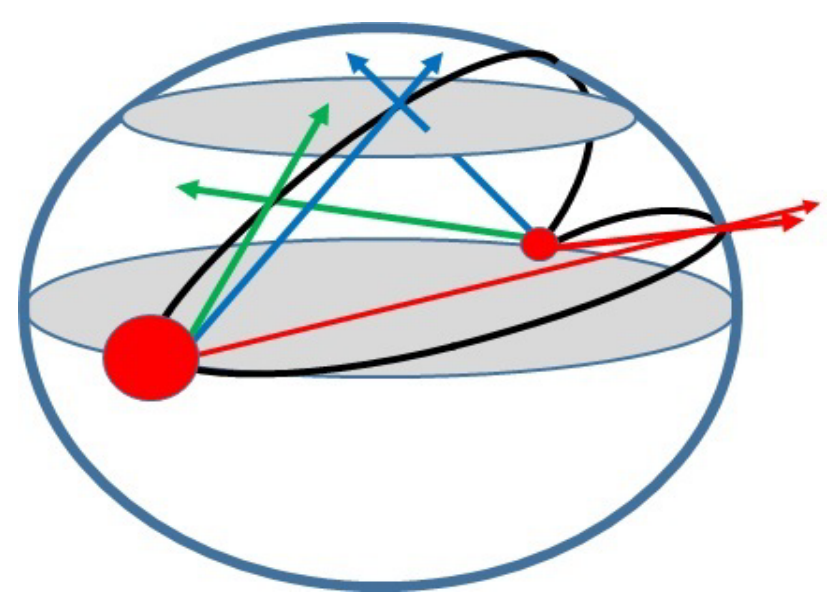

Figure 22: Why the contribution of each zone for the production of the total force following the new Coulomb law $F=K Q q / d^{\mathrm{x}}$ is proportional to the area of their surfaces.

the quantity of SCi interactions. As the reduction of the area is directly proportional to the decrease of the square of the distance $d$, then when $d$ is shorter than $30 \mathrm{fm}$ occurs a phenomenon "inverse" of what occurs for distances d larger than $30 \mathrm{fm}$. Let us call it "Inverse Coulomb" phenomenon.

Then let us to begin by explaining the procedure for calculation of Coulomb forces, as follows. a. In Excel is calculated the old Coulomb force of repulsion $F_{R}=K Q q / d^{2}$, where $K=9 \times 10^{9}$, $\mathrm{Q}$ and $\mathrm{q}$ are the charges of $\mathrm{U} 238$ and $\mathrm{He} 4$, respectively $92 \times 1,6 \times 10^{-19} \mathrm{C}$ and $2 \times 1,6 \times 10^{-19}$ $C$, the distance " $d$ " begins to increase in $4 \times 10^{-15} \mathrm{~m}$, and two consecutive points of the graphic are separated by a distance $\Delta d=$ $2 \times 10^{-15} \mathrm{~m}$.

b. The next step is to calculate the percentages 
of contribution of each one of the three zones, for the production of the total force, they following the new Coulomb law $F=$ $K Q q / d^{x}$, as explained in the item $c$ ahead.

c. The contribution of the force in a zone is proportional to the area of its surface. The reason why it is proportional do the area of the surface can be understood by looking at the Figure 22, where it is seen that, whereas the red and green strings interact in the medium zone, in the case of the two blue orthogonal strings the situation is different, because in spite of $90 \%$ of their lengths are situated in the medium zone, however they have interaction in the optimum zone. In another words, although the two blue strings travel along $90 \%$ of their trajectory inside the "volume" of the medium zone (where there is not any $\mathrm{SCi}$ ), however they interact in the "surface" of the cap of the optimal zone. So, the contribution of each zone is proportional to the area of its surface. d. The areas of the three zones are: $2 \pi R$. $(0.1 R)=0.2 \pi R^{2}$ for the optimal zone. As the total area of the cap is $2 \pi R^{2}$, then the contribution of the optimal zone is 0.1 . For the medium zone, the area of the surface is $2 \pi R \cdot(0.7 R-0.1 R)=1.2 \pi R^{2}$, and so the contribution of the medium zone is 0.6. Finally, the contribution of the critical zone is $1-(0.1+0.6)=0.3$.

e. The force in the optimal zone is calculated as follows:

$$
F_{o p}=0.1 \cdot \mathrm{F}_{\mathrm{R}} \cdot\left(\Delta S_{o p}\right)^{\lambda o p}
$$

where:

- $F_{R}$ was calculated in the item "a" above

- $\Delta S_{o p}$ is the growth of the surface $2 \pi R h$ of the cap

- $\lambda_{\text {op }}$ is $10^{u} \cdot\left[10^{-15} /\left(2 \times 10^{-15}+\Delta d\right)\right]^{2}$ of the density of strings, in the optimal zone (see Figure 19).

$F_{O P}$ is typed in the line 6 of the Excel in the Table 2, $F_{M E D}$ in the line 12 , and $F_{C R}$ in the line 18. The equations typed in Excel are seen in the Table 3.

Table 2: Calculation of the contribution of each of the three SCi forces. The red curve is the contribution of $F_{\text {MED }}$ in medium zone, and it is seen that it collapses strongly between the points $1.8 \mathrm{E}-14$ and $6 \mathrm{E}-15$. The blue curve for $F_{O P}$ in the optimal zone collapses not so strongly, between $1.2 \mathrm{E}-14$ and $6 \mathrm{E}-15$. The green curve for critical force $F_{C R}$ collapses slowly between $1.8 \mathrm{E}-14$ and $4 \mathrm{E}-14$. Note that the curves begin to be plot in the distance $4 \mathrm{fm}$ (the distance $2 \mathrm{fm}$, in the cell B2, is used only as a way to establish the step of the growth of the distances: $\Delta d=2 \mathrm{E}-15$ ).

\begin{tabular}{|c|c|c|c|c|c|c|c|c|c|c|c|c|c|}
\hline 4 & A & B & C & D & $E$ & $F$ & G & $\mathrm{H}$ & 1 & J & K & L & M \\
\hline 1 & & & 1 & 2 & 3 & 4 & 5 & 6 & 7 & 8 & \multirow{2}{*}{$2 E-14$} & 10 & 11 \\
\hline 2 & $d=R=$ & $1 \mathrm{E}-15$ & 3E-15 & $5 E-15$ & $7 \mathrm{E}-15$ & $9 \mathrm{E}-15$ & $1 \mathrm{E}-14$ & $1 \mathrm{E}-14$ & $2 \mathrm{E}-14$ & $2 E-14$ & & $2 \mathrm{E}-14$ & $2 \mathrm{E}-14$ \\
\hline 3 & $\lambda_{\mathrm{OP}}=$ & & 38,66667 & 13,92 & 7,10204 & 4,2963 & \multirow{2}{*}{$\begin{array}{l}2,87603 \\
1.1 F-1.5\end{array}$} & 2,05917 & 1,54667 & 1,20415 & 0,96399 & 0,78912 & 0,65784 \\
\hline 4 & $h=$ & $1 \mathrm{E}-16$ & $3 E-16$ & $5 E-16$ & $7 E-16$ & $9 \mathrm{E}-16$ & & $1.3 F-1.5$ & $1.5 \mathrm{~F}-1.5$ & 1.7F-1.5 & $1.9 F-1.5$ & $2.1 \mathrm{~F}-1.5$ & $2.3 F-15$ \\
\hline 5 & $\mathrm{~S}_{\mathrm{OP}}=$ & $6,28 \mathrm{E}-31$ & $5,65 \mathrm{E}-30$ & $1,6 \mathrm{E}-29$ & $3,1 \mathrm{E}-29$ & $5 \mathrm{E}$ & & & & & & & 8 \\
\hline 6 & $F=K Q q / d^{x}$ & & $5,97 \mathrm{E}-35$ & 0,00011 & 0,72693 & & & & & & & & \\
\hline 7 & $F=K Q q / d^{2}$ & & 471,04 & 169,574 & 86,5176 & $52, z^{4}$ & & & & & & & \\
\hline 8 & & & & & & & & & & & & & \\
\hline 9 & $\lambda_{\text {MED }}=$ & & 89,55556 & 32,24 & 16,449 & $9,9 \underbrace{}_{30}$ & & & & & & & \\
\hline 10 & $h=$ & $6 \mathrm{E}-16$ & $1,8 \mathrm{E}-15$ & $3 \mathrm{E}-15$ & $4,2 \mathrm{E}-15$ & $5 \mathrm{E} \cdot 25$ & & & & & & & \\
\hline 11 & $\mathrm{~S}_{\text {MED }}=$ & $3,768 \mathrm{E}-30$ & $3,39 \mathrm{E}-29$ & $9,4 \mathrm{E}-29$ & $1,8 \mathrm{E}-28$ & $3 \mathrm{E} \cdot{ }_{20}$ & & & & & & & 7 \\
\hline 12 & $F=K Q q / d^{x}$ & & $9,85 E-83$ & $5 \mathrm{E}-12$ & 0,00809 & $2,11_{15}$ & & & & & & & \\
\hline 13 & $\mathrm{~F}=\mathrm{KQq} / \mathrm{d}^{2}$ & & 2826,24 & 1017,45 & 519,105 & $314{ }_{10}$ & & & & & & & \\
\hline 14 & & & & & & & & & & & & & \\
\hline 15 & $\lambda_{\mathrm{CR}}=$ & & 1111,111 & 400 & 204,082 & 1230 & & & & & & & \\
\hline 16 & $h=$ & $3 \mathrm{E}-16$ & $9 \mathrm{E}-16$ & $1,5 \mathrm{E}-15$ & $2,1 \mathrm{E}-15$ & $3 \mathrm{E}$ & $\begin{array}{llll}12 & 2 & 4\end{array}$ & $\begin{array}{llll}4 & 5 & 6 & 7\end{array}$ & 89101 & 111213141 & 15161718 & 181920212 & 2223 \\
\hline 17 & $\mathrm{~S}_{\mathrm{CR}}=$ & $1,884 \mathrm{E}-30$ & $1,7 \mathrm{E}-29$ & $4,7 E-29$ & $9,2 E-29$ & $2 E-28$ & $2,3 \mathrm{E}-28$ & $3,2 \mathrm{E}-28$ & $4,2 E-28$ & $5,4 \mathrm{E}-28$ & $6,8 \mathrm{E}-28$ & $8,3 \mathrm{E}-28$ & $1 E-27$ \\
\hline 18 & $F=K Q q / d^{x}$ & & 0 & $2 \mathrm{E}-175$ & $5,9 \mathrm{E}-58$ & $2 \mathrm{E}-25$ & 4,1E-13 & $2 \mathrm{E}-07$ & 0,00017 & 0,00762 & 0,07426 & 0,30814 & 0,77136 \\
\hline 19 & $\mathrm{~F}=\mathrm{KQq} / \mathrm{d}^{2}$ & & 1413,12 & 508,723 & 259,553 & 157,01 & 105,108 & 75,2549 & 56,5248 & 44,0072 & 35,2301 & 28,8392 & 24,0417 \\
\hline
\end{tabular}


Table 3: The equations used for calculations in Excel, for the three zones.

\begin{tabular}{|c|c|c|c|c|}
\hline$\Delta$ & A & B & $\mathrm{C}$ & $\mathrm{D}$ \\
\hline 1 & & & 1 & 2 \\
\hline 2 & $\mathbf{d}=\mathbf{R}=$ & $2 * 10^{\wedge}-15$ & $B 2+2 * 10^{\wedge}-15$ & \\
\hline 3 & $\boldsymbol{\lambda}_{\mathrm{OP}}=$ & \multirow{5}{*}{$\begin{array}{c}=0,1{ }^{*} \mathrm{~B} 2 \\
2 * 3,14{ }^{*} \mathrm{~B} 2{ }^{*} \mathrm{~B} 4\end{array}$} & $348^{*}\left(\left(10^{\wedge}-15 / \mathrm{C} 2\right)^{\wedge} 2\right)$ & \\
\hline 4 & $\mathbf{h}=$ & & $0,1 * \mathrm{C} 2$ & \\
\hline 5 & $\mathbf{S}_{\mathrm{OP}}=$ & & $2 * 3,14 * \mathrm{C} 2 * \mathrm{C} 4$ & \\
\hline 6 & $F=K Q q / d^{X}$ & & $C 7^{*}\left(\left((B 5 / C 5)^{\wedge} C 3\right)\right)$ & \\
\hline 7 & $F=K Q q / d^{2}$ & & $0,1^{*}\left(9^{*} 10^{\wedge} 9\right)^{*}\left(92^{*} 1,6^{*} 10^{\wedge}-19\right)^{*}\left(2^{*} 1,6^{*} 10^{\wedge}-19\right) /\left((C 2)^{\wedge} 2\right)$ & \\
\hline \multicolumn{5}{|l|}{8} \\
\hline 9 & $\boldsymbol{\lambda}_{\mathrm{MED}}=$ & \multirow{5}{*}{$\begin{array}{c}0,6^{*} \mathrm{~B} 2 \\
22^{*}, 14^{*} \mathrm{~B} 2{ }^{*} \mathrm{~B} 10\end{array}$} & $806^{*}\left(\left(10^{\wedge}-15\right) / C 2\right)^{\wedge} 2$ & \multirow{5}{*}{$\begin{array}{c}\text { copy } \\
\text { to } \\
\text { other } \\
\text { colunes }\end{array}$} \\
\hline 10 & $\mathbf{h}=$ & & $0,6 * \mathrm{C} 2$ & \\
\hline 11 & $\mathbf{S}_{\mathrm{MED}}=$ & & $2 * 3,14 * \mathrm{C} 2 * \mathrm{C} 10$ & \\
\hline 12 & $F=K Q q / d^{x}$ & & $\mathrm{C} 13^{*}\left((\mathrm{~B} 11 / \mathrm{C} 11)^{\wedge} \mathrm{C} 9\right)$ & \\
\hline 13 & $F=K Q q / d^{2}$ & & $0,6^{*}\left(9^{*} 10^{\wedge} 9\right)^{*}\left(92^{*} 1,6^{*} 10^{\wedge}-19\right)^{*}\left(2^{*} 1,6^{*} 10^{\wedge}-19\right) /\left((C 2)^{\wedge} 2\right)$ & \\
\hline \multicolumn{5}{|c|}{ : } \\
\hline 15 & $\boldsymbol{\lambda}_{\mathrm{CR}}=$ & \multirow{5}{*}{$\begin{array}{c}0,3^{*} \mathrm{~B} 2 \\
2 * 3,14^{*} \mathrm{~B} 2{ }^{*} \mathrm{~B} 16\end{array}$} & $10^{\wedge} 4^{*}\left(10^{\wedge}-15 / \mathrm{C} 2\right)^{\wedge} 2$ & \\
\hline 16 & $\mathbf{h}=$ & & $0,3^{*} \mathrm{C} 2$ & \\
\hline 17 & $\mathrm{~S}_{\mathrm{CR}}=$ & & $2 * 3,14 * \mathrm{C} 2 * \mathrm{C} 16$ & \\
\hline 18 & $F=K Q q / d^{x}$ & & $\mathrm{C} 19^{*}\left((\mathrm{~B} 17 / \mathrm{C} 17)^{\wedge} \mathrm{C} 15\right)$ & \\
\hline 19 & $F=K Q q / d^{2}$ & & $0,3^{*}\left(9^{*} 10^{\wedge} 9\right)^{*}\left(92^{*} 1,6^{*} 10^{\wedge}-19\right)^{*}\left(2^{*} 1,6^{*} 10^{\wedge}-19\right) /\left((C 2)^{\wedge} 2\right)$ & \\
\hline
\end{tabular}

Table 4: New calculation of ratio between the repulsion calculated by Rutherford and the real repulsion between U238 and He4, by comparing the old Coulomb force $F=K Q q / d^{2}$ with the new Coulomb force $F=K Q q / d^{x}=F_{O P}+F_{M E D}+F_{C R}$.

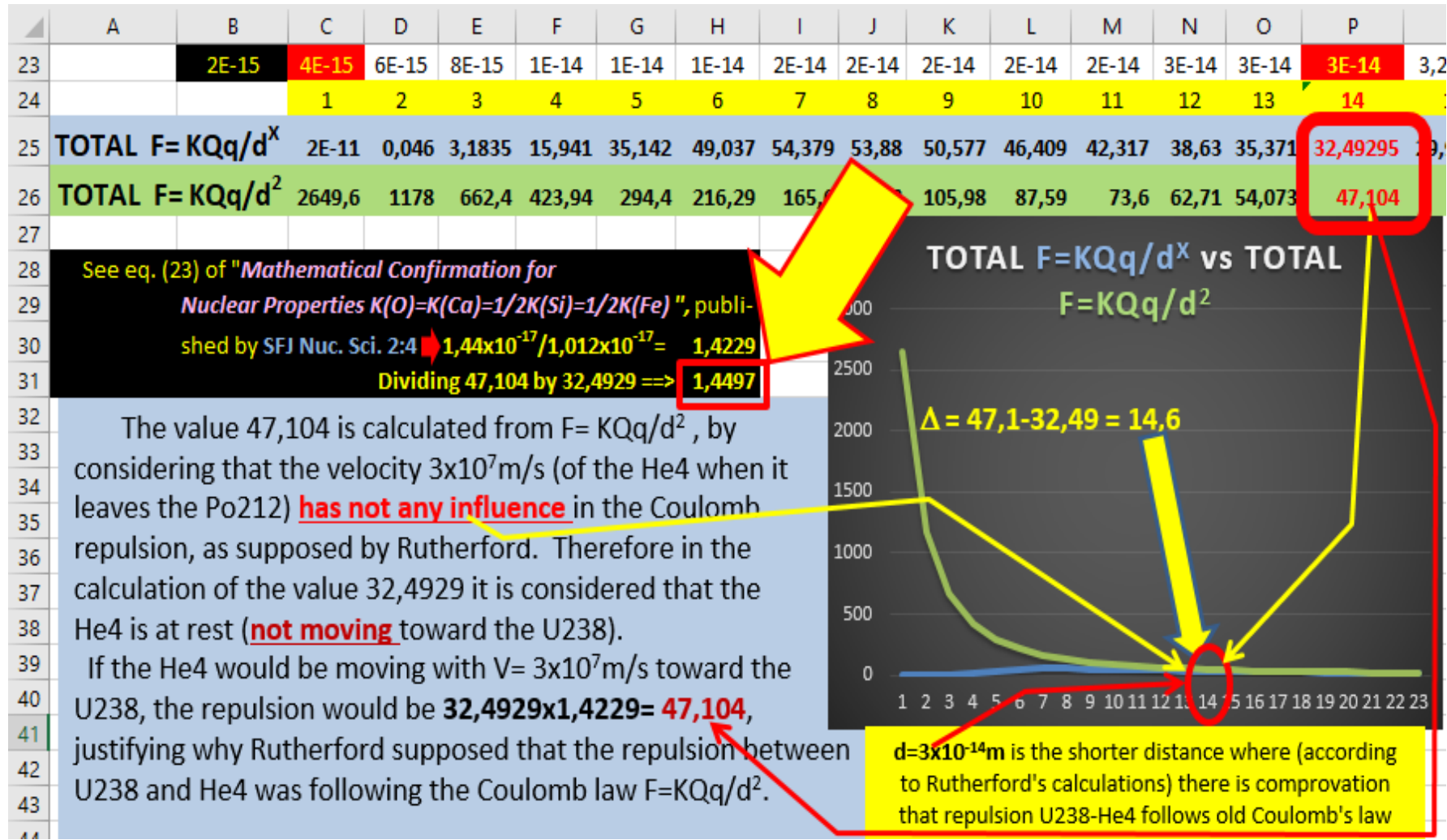

Two Different Procedures of Calculation Giving the Same Result

In this present paper was shown that Gamow's paradox can be solved by using eq. (3) and (4), a task performed in the paper [5].

- One particle moving with speed V toward other particle at rest 


$$
\Delta(\mathrm{sw})=\left[\left(\mathrm{c}+\left(\frac{\mathrm{Q}}{\mathrm{e}}\right) \cdot \mathrm{V}\right)^{2}\right]^{\left(1+\frac{\mathrm{d}}{\mathrm{\phi}}\right)}
$$

- One particle moving with speed $\mathrm{V}$ leaving a nucleus

$$
\Delta(\mathrm{sw})=\left[\left(\mathrm{c}-\left(\frac{\mathrm{Q}}{\mathrm{e}}\right) \cdot \mathrm{V}\right)^{2}\right]^{\left(1+\frac{d}{\Phi}\right)}
$$

The other procedure, used in the paper [8], gives the same result, where the new starting point is the eq. (36), previously mentioned here:

$$
F_{o p}=0.1 \cdot \mathrm{F}_{\mathrm{R}} \cdot\left(\Delta S_{o p}\right)^{\lambda o p}
$$

Table 4 shows what happens in the point $\mathrm{R}=$ $3 \mathrm{E}-14$, using the eq. (36). The aim was to reach to the ratio between the repulsion calculated by Rutherford and the real repulsion between U238 and $\mathrm{He} 4$ (moving toward U238), from the two different procedures ahead.

- From a procedure using the eq. (36) for the calculation of the ratio between the old Coulomb force $F=K Q q / d^{2}$ and the new Coulomb's force $\sum F\left[\frac{K Q q}{d X}\right]=F_{O P}+F_{M E D}+F_{C R}$, calculated in the paper [8]. It's seen at Table 4 that the ratio is 1.449 .

- From the procedure using eq. (3) and (4) in the calculus made in the paper [5]. The ratio is 1.4229 .

In the graphic at Table 4, the green curve is the old Coulomb force, and the blue curve is the total contribution of the three forces in the three zones, $\sum F=F_{O P}+F_{M E D}+F_{C R}$.

\section{Troubles in Hartree-Fock Methods}

\section{Rose-Bethe calculations}

Hartree-Fock method [16] has failed for the calculation of magnetic moments for some nuclides, as seen in Table 5. In particular, the wrong prediction of nuclear spin for B10 (with five protons and five neutrons) contributes for reinforcing the need of considering a new nuclear model, but with some new principles missing in the current Nuclear Physics, as exposed ahead.

- Consider that all nuclei have a central $2 \mathrm{He} 4$, around which deuterons are distributed. In the case of B10, the three deuterons captured by the central $2 \mathrm{He} 4$ align their spins, giving to boron-10 the spin 3+. As in Hartree-Fock method it is not considered a central $2 \mathrm{He} 4$, this is the reason why it has failed in the prediction of its nuclear spin. Because without considering a central $2 \mathrm{He} 4$, we have to expect a structure of B10 with spin 1, as predicted by Rose-Bethe calculation. As consequence, they also calculated a wrong magnetic moment, only $47 \%$ of the experimental value +1.80 . And the reason of the large difference $\Delta$ $=1.80-0.85=0.95$ is because, due to the rotation of the nucleus, the rotation of the electric charges of the three protons induce a big magnetic moment, which is not considered in the Rose-Bethe calculation. Boron-10 is the unique nucleus, with $Z<8$, where three deuterons interact with aligned spins, surrounding a central $2 \mathrm{He} 4$, and that's why Hartree-Fock method fails drastically. There are other nuclei with three deuterons with their spins aligned, but all them have $\mathrm{Z}>8$ (as for instance $\mathrm{Na22}$ ) and their structures are completely different, because starting from oxygen isotopes the structures of nuclei

Table 5: Rose-Bethe calculation of magnetic moments and spins through Hartree-Fock Methods (1937).

\section{Calculated nuclear magnetic moments and spins.}

\begin{tabular}{|l|l|l|l|l|l|}
\hline Nucleus & Mag. Mom. & Spin & Nucleus & Mag. Mom. & Spin \\
\hline $\mathrm{Li}^{6}$ & 0.85 & 1 & $\mathrm{~B}^{11}$ & 3.50 & $3 / 2$ \\
\hline $\mathrm{Li}^{7}$ & 3.15 & $3 / 2$ & $\mathrm{~B}^{12}$ & 1.73 & 2 \\
\hline $\mathrm{Li}^{8}$ & 0.97 & 2 & $\mathrm{C}^{11}$ & -1.65 & $3 / 2$ \\
\hline $\mathrm{Be}^{7}$ & -1.30 & $3 / 2$ & $\mathrm{C}^{13}$ & 1.13 & $1 / 2$ \\
\hline $\mathrm{Be}^{9}$ & -1.65 & $3 / 2$ & $\mathrm{~N}^{13}$ & -0.75 & $1 / 2$ \\
\hline $\mathrm{B}^{9}$ & 3.50 & $3 / 2$ & $\mathrm{~N}^{14}$ & 0.85 & 1 \\
\hline $\mathrm{B}^{10}$ & 0.85 & 1 & $\mathrm{~N}^{15}$ & -0.28 & $1 / 2$ \\
\hline & & & $\mathrm{O}^{15}$ & 0.67 & $1 / 2$ \\
\hline
\end{tabular}




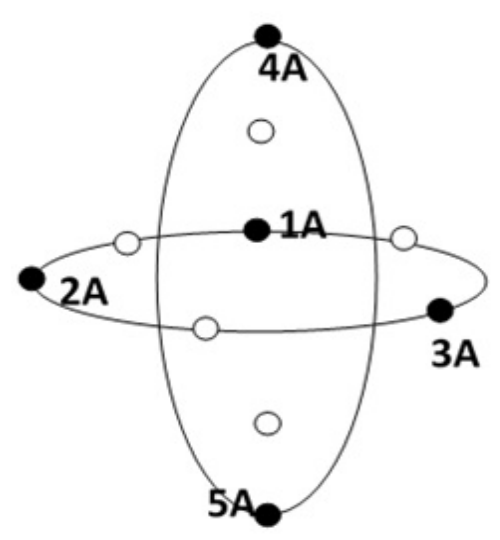

5B10

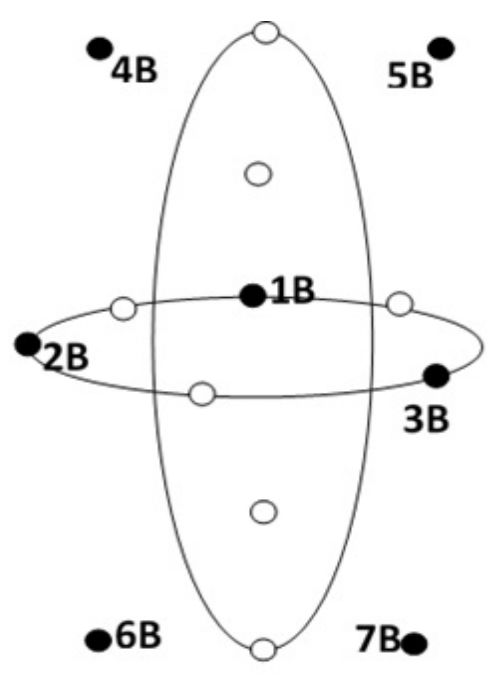

7N14

Figure 23: Structures for 5B10 and 7N14, as expected from the principles of the current Nuclear Physics, by considering repulsion by traditional Coulomb law and attraction by strong nuclear force.

have a drastic change.

- From the model with a central $2 \mathrm{He} 4$ is calculated successfully the magnetic moment for B10, whose structure is shown in the Figure 35 at the page 28 of [4]. The calculation in the Figure 36 shows that, without considering the rotation, B10 would have $\mu(B 10)=0.504$. The same happens with the others nuclei having odd protons, as for instance N14, seen in Figure 33. But the contribution of rotation for $\mu(\mathrm{N} 14)$ is very smaller than in the case of B10, because while B10 has three unpaired deuterons, N14 has only one unpaired deuteron, and also because the angular velocity $\omega$ of its rotation is slower than in B10 (for N14, $\omega=0.02753$, seen in the eq. 127 , and for B10 $\omega=0.04824$, seen in eq. 142). The relation between the PWR and the angular velocity of two nuclei $\mathrm{A}$ and $\mathrm{B}$ is $\frac{P w R(A)}{P w R(B)}=\frac{\omega(A)}{\omega(B)}$.

- Bethe had estimated that $10 \%$ of the nuclear magnetic moment is due to the contribution of the rotation of the nuclei, responsible for the difference between the calculation from Hartree-Fock method and the experimental value. But he could not imagine that, in the case of some nuclei with three unpaired protons, the contribution can reach to the order of $150 \%$.

- Some nuclear properties are missing in the current Nuclear Physics, because they were not discovered by nuclear theorists. For instance, that silicon and iron isotopes have induction-factors twice of that for oxygen and calcium isotopes. Then it is obvious that it is impossible from the current nuclear models to calculate the magnetic moments for many exotic isotopes, no matter what sort of arbitrary assumptions a nuclear theorist may desperately to adopt.

\section{Impossible electric quadrupole moment for 5B10}

Several exotic nuclei have nuclear properties impossible to be explained from the current principles of the Nuclear Physics. Concerning the electric quadrupole moments $\mathrm{Q}(\mathrm{B} 10)$ and $\mathrm{Q}(\mathrm{N} 14)$ of the isotopes 5B10 and 7N14, ahead is proven to be impossible for $Q(B 10)$ to be 4.2 times larger than $Q(N 14)$. But the experiments have detected that $\mathrm{Q}(\mathrm{B} 10)=4.2 \mathrm{Q}(\mathrm{N} 14)$.

On the reasons why electric quadrupole moment $Q(5 B 10)$ cannot be 4.2 times greater than $\mathbf{Q}(\mathbf{7 N 1 4 ) :}$ Figure 23 shows the distribution of protons and neutrons, that we have to expect to be, in the structures of the atomic nuclei $5 \mathrm{~B} 10$ and $7 \mathrm{~N} 14$, by considering the foundations of the Standard Nuclear Physics, and of course considering that protons-protons, neutrons-neutrons, and protons-neutrons, are bound via strong force, and protons-protons have repulsion through the old traditional Coulomb's Law.

Note that, in $7 \mathrm{~N} 14$, the distance of the two 

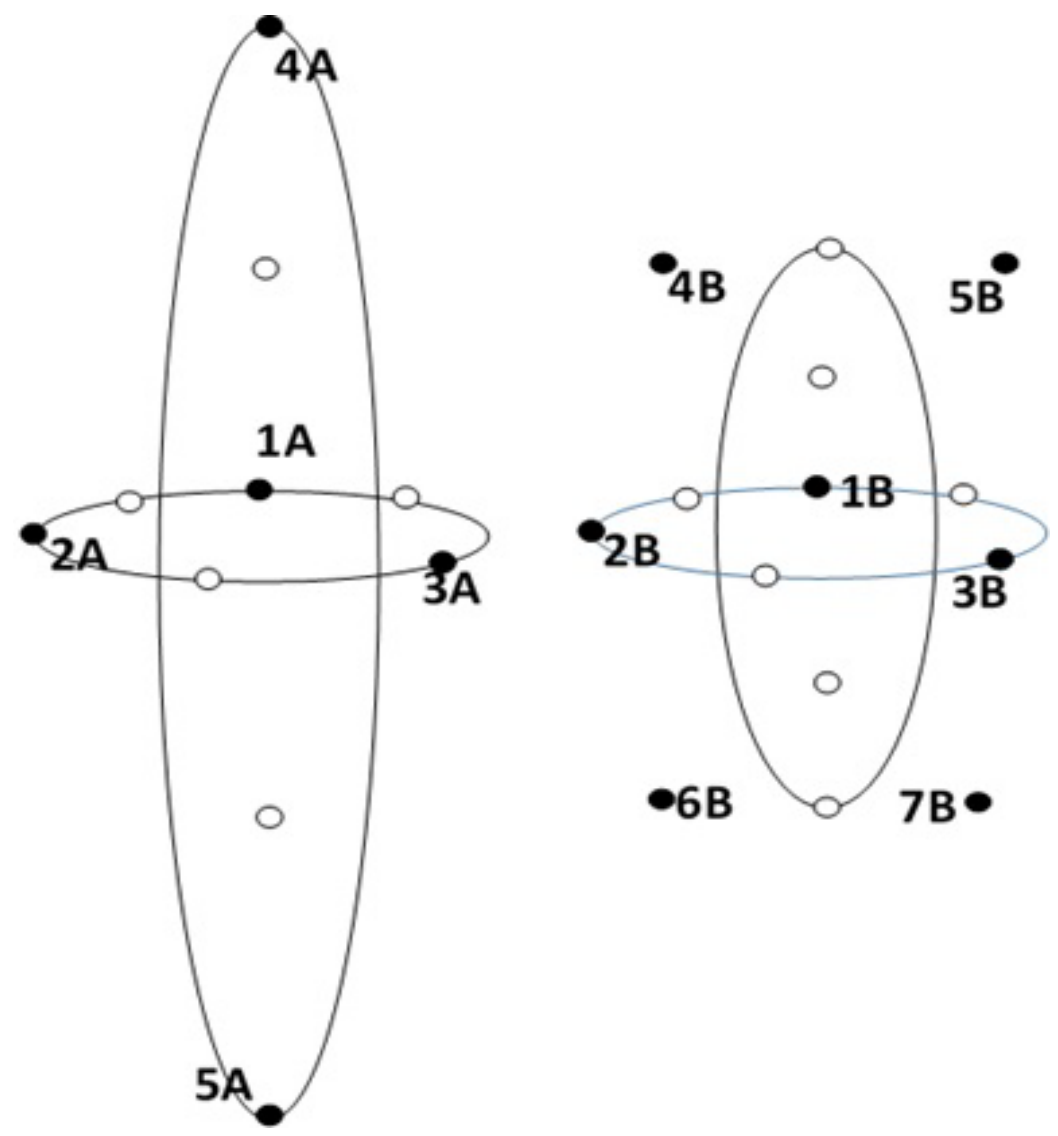

Figure 24: The structures of B10 and N14 as they have to be, by considering that experiments detected the relation $\mathrm{Q}(\mathrm{B} 10)=4.2 \mathrm{Q}(\mathrm{N} 14)$.

protons $4 \mathrm{~B}$ and $5 \mathrm{~B}$ to the center of the nucleus is larger than the distance of the proton $4 \mathrm{~A}$ to the center of 5B10. The reason is because the Coulomb repulsion between $4 B$ \& $5 B$ and the central protons $1 \mathrm{~B}-2 \mathrm{~B}-3 \mathrm{~B}$, in $7 \mathrm{~N} 14$, is stronger than the repulsion between $4 A$ and the central protons $1 A-2 A-3 A$, in 5B10. So, from the structure predicted from the foundations of the Standard Nuclear Physics, we have to expect that 7N14 needs to have an electric quadrupole moment $\mathrm{Q}(\mathrm{N} 14)$ a little larger than Q(B10):

$$
\mathrm{Q}(\mathrm{N} 14)>\mathrm{Q}(\mathrm{B} 10)
$$

But the experiments have measured the following values, in barns.

$$
\begin{aligned}
& \mathrm{Q}(\mathrm{B} 10)=+0.084 \\
& \mathrm{Q}(\mathrm{N} 14)=+0.020
\end{aligned}
$$

Therefore, $Q(B 10)$ is 4.2 times larger than $\mathrm{Q}(\mathrm{N} 14)$. Then, by considering their electric quadrupole moments measured in the experiments, we have to consider a structure for $5 \mathrm{~B} 10$ as shown in the Figure 24.
But from the foundations of the Standard Nuclear Physics, it's impossible a structure for 5B10 as shown in Figure 24.

As seen in the Rose-Bethe calculation at the Table 5, their theory predicted a spin 1 for 5B10, and here is the origin of the error. Such prediction of spin 1 for boron-10 is due to the fact that, from the sort of interactions expected from the principles of the Nuclear Physics, the distribution of nucleons in 5B10 must be similar to that in 7N14. Therefore, if Nuclear Physics was correct, 5B10 would have a structure like seen in the Figure 23 , having $Q(B 10)$ a little smaller than $Q(N 14)$. So, there is no any doubt that the calculations based on the Hartree-Fock Methods fail because there is not a central $2 \mathrm{He} 4$ in the current nuclear models. Often it fails for the prediction of magnetic moments, nuclear spins, and quadrupole moments. But to say that Hartree-Fock Methods are unable to predict quadrupole moments is very little, because as already was showed in this paper, it's impossible to explain why the ratio of quadrupole moments $Q(B 10) / Q(N 14)=$ 4.2. And this is no small thing. One thing is when a 


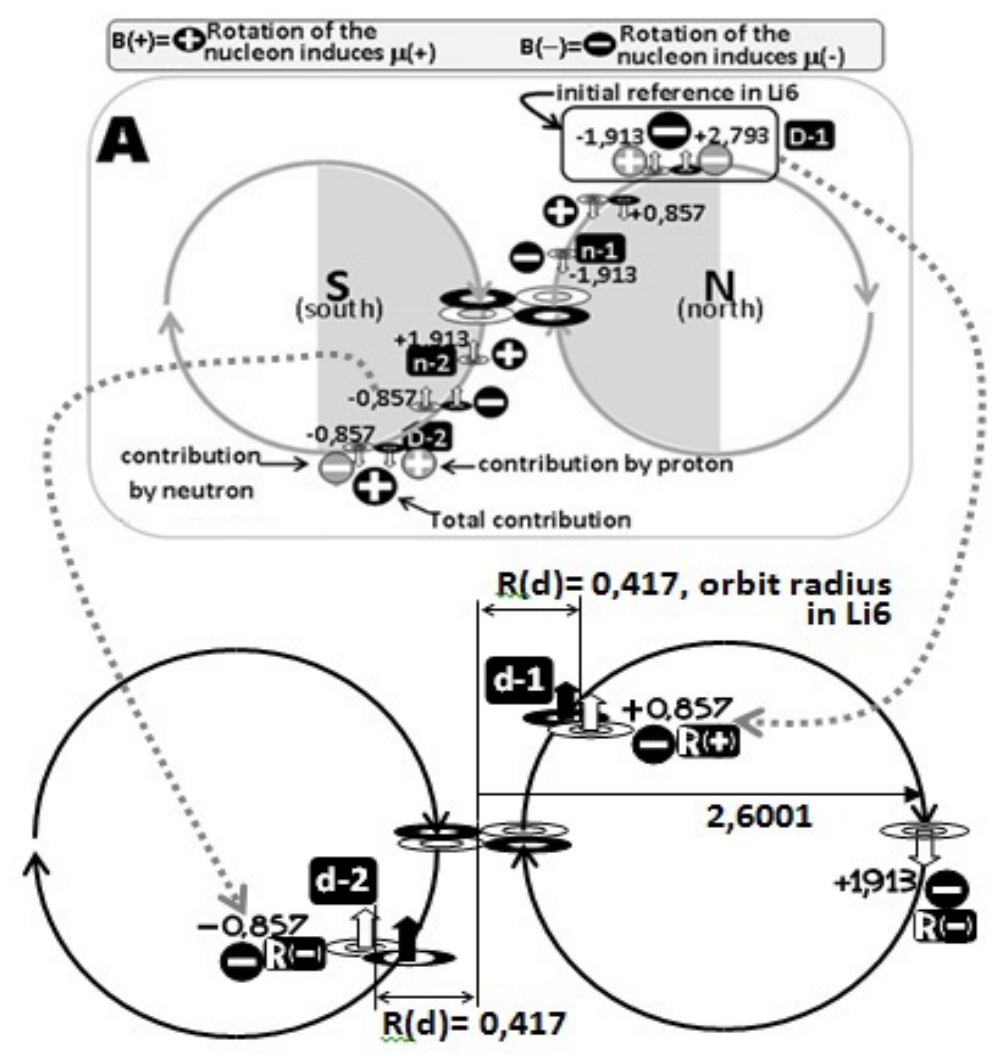

Figure 25: The structure of $4 \mathrm{Be} 9$ has $Q(B e 9)=+0.0529$.
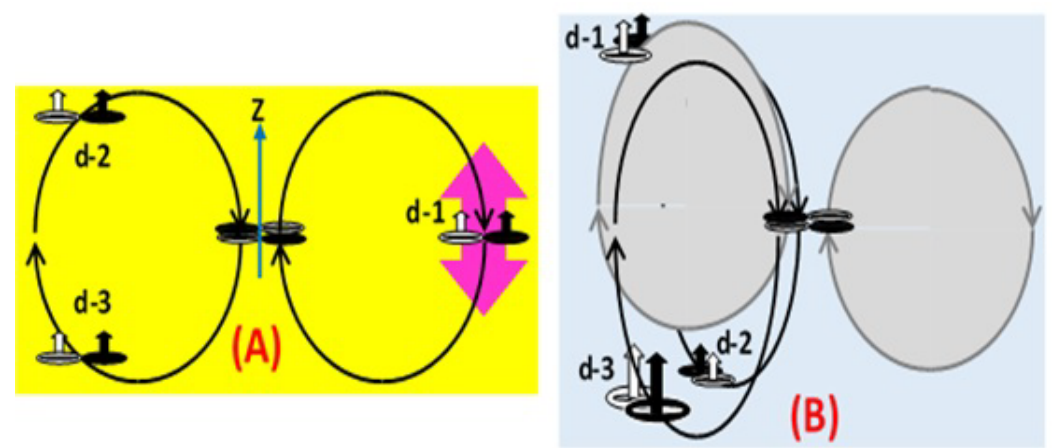

Figure 26: Distribution of the three deuterons in $5 B 10,3+$, with $Q(B 10)=+0.0845$.

theory cannot predict correctly the value of something whose correct value however is no impossible, according to the theory. Other thing completely different is when a theory cannot predict correctly the value of something, whose correct value is impossible, according to the theory. And finally note that, as even by using a dirty mathematics it's impossible to explain the puzzle why $Q(B 10)=4.2 \times$ (N14), then we realize that the foundations of the current Nuclear Physics cannot be correct.

\section{The impossible 5B10 with spin 3+}

Hartree-Fock Methods fail to explain the spin of $5 B 10$, and ahead it is explained the reason why it has spin $3+$, in spite of it is impossible from the principles of the current Nuclear Physics.

Let us understand the structure of 5B10, by comparing it with that of $4 \mathrm{Be} 9$.

The structure of $4 \mathrm{Be} 9$ is seen in the Figure 25, which is a reproduction of the Figure 39 of the paper [4]. Concerning the length of the orbit radii of the two deuterons in 4Be9, if the deuteron $\mathrm{d}-1$ were alone, it would slide along the $\mathrm{n}(\mathrm{o})$-flux, pulled by the centrifugal force of the neutron, applied on it, as occurs with the deuteron in $3 \mathrm{Li} 7$, and 

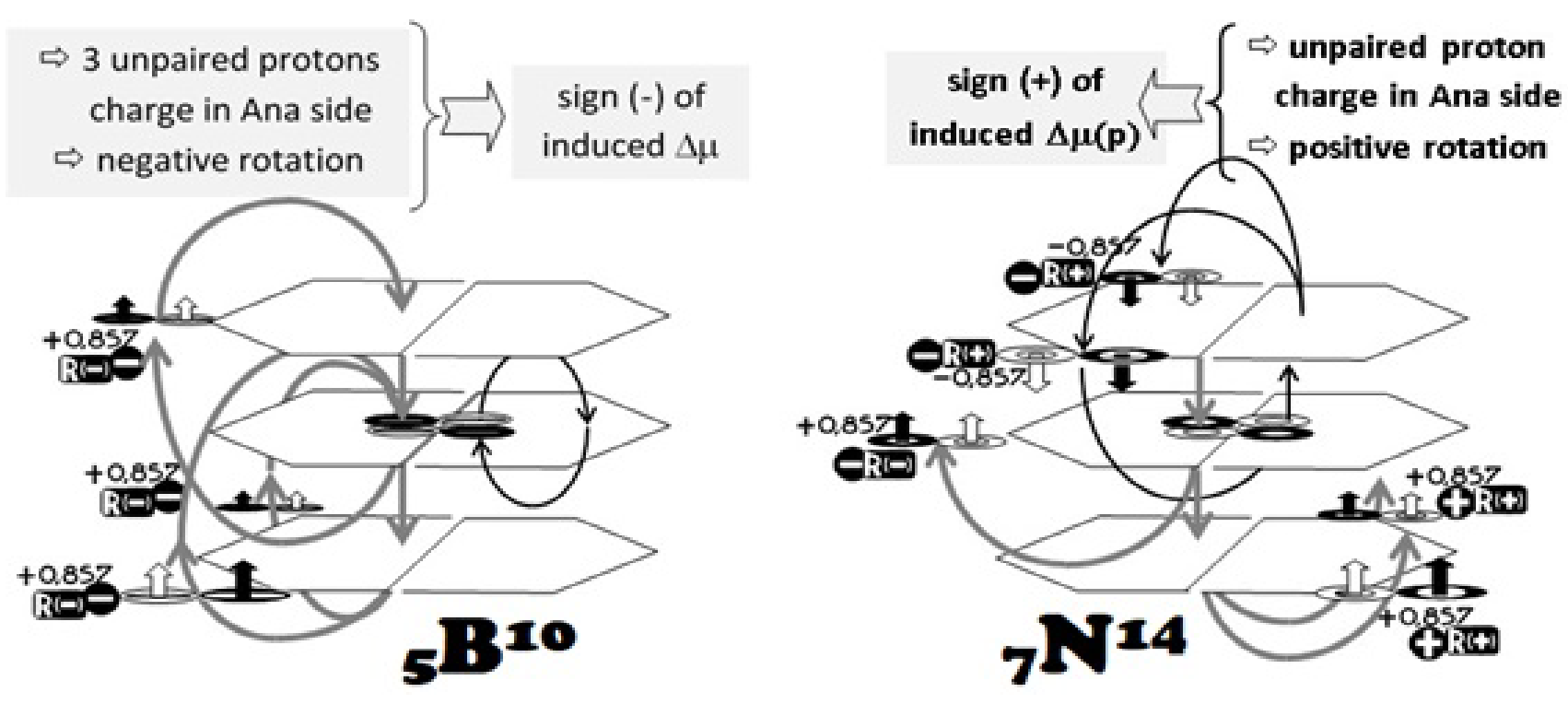

Figure 27: Structure for $5 \mathrm{~B} 10$ from which was calculated $\mu(B 10)=+1.918 \mu \mathrm{N}$. The "induced $\Delta \mu$ " seen in the figure is referred to the magnetic moment induced by the rotation (around the central 2He4) of the charges of the three unpaired deuterons situated in the Ana side, while the "sing (-)" indicates that the negative total rotation of the nucleus (with the three deuterons in the Ana side) induces an additional negative magnetic moment.

therefore the orbit radius $\mathrm{R}(\mathrm{d})$ of $\mathrm{d}-1$ in $\mathrm{Be} 9$ would be larger than $R(d)=0.417 \mathrm{fm}$ in $3 \mathrm{Li6}$. But the force of interaction between the two deuterons prevents the slide of the $d-1$ (and $d-2$ too). Therefore, it is reasonable to suppose that the value of $R(d)$ in Be9 is approximately the same value $R(d)=0.41689$ in lithium-6, calculated in the eq. (163) of the paper [4]. That's why $R(d)=0.41689$ will be used in the calculation for $\mu$ (Be9).

Magnetic moment $\mu(4 \mathrm{Be} 9)$ is calculated from $\mathrm{K}_{\mathrm{TH}}(\mathrm{Be} 9)$, as follows.

$$
\begin{aligned}
\mathrm{K}_{\mathrm{TH}}(\mathrm{X}) & =\left(\frac{\Phi}{6}\right) \cdot 1,37176 \cdot \frac{\operatorname{PwR}(\mathrm{X}) \cdot \mathrm{R}(\mathrm{X})}{\operatorname{PwR}(015) \cdot \mathrm{R}(015)} \\
\mathrm{R}(\mathrm{Be} 9) & =1.25 \times 9^{1 / 3}=2.6001
\end{aligned}
$$

The two deuterons cancel their $R(+)$ and $R(-)$, and then $\mathrm{PwR}(\mathrm{Be} 9)$ is produced by the neutron, whose contribution is negative, $R(-)$ :

$\operatorname{PwR}($ Be9 $)=2.6021 /\left(9 \times 2.6001^{2}\right)=0.0427656(38)$,

where 2.6021 is the neutron $\mathrm{PwC}(n)=2.6021$, seen in eq. (17) of this paper.

As $\Phi=2$ for Be9 and $R(d)=0.41689$ :

$$
\mathrm{K}_{\text {TH }}(\mathrm{Be} 9)=1.371761^{2 / 6} \times 0.41689 \times 0.0427656 /
$$

$(3.08276 \times 0.03179)=0.206763$

From the structure for $\mathrm{Be} 9$ in the Figure 25:
$\mu(\mathrm{Be} 9)=+1.913+0.857-0.857+0.206763($

$-1.913-2 \times 0.857)$

$\mu(\mathrm{Be} 9)=+1.1629 \mu \mathrm{N}$

As $\mathrm{Be} 9$ has negative rotation, then:

$\mu(\mathrm{Be} 9)=-1.1629 \mu \mathrm{N}$

and the experimental value is $\mu(\mathrm{Be} 9)=$ $-1.1778(9) \mu N$.

So, the successful calculation of $\mu(B e 9)$ suggests us that we can consider for Be9 the structure shown in Figure 25. Then, looking at the distribution of proton charges in its structure, we realize that the quadrupole moment $\mathrm{Q}(\mathrm{Be} 9)$ is agree to the experimental value $\mathrm{Q}(\mathrm{Be})=+0.0529$ barns. So, everything is very well with the theoretical structure for $4 \mathrm{Be} 9$, according to the Hexagonal Floors model.

But suppose that the neutron is removed from the $4 \mathrm{Be} 9$. Then in then newborn $4 \mathrm{Be} 8$ the two deuterons have attraction through spin-interaction and move toward the central $2 \mathrm{He} 4$, and $4 \mathrm{Be} 8$ decays. The stability of $4 \mathrm{Be} 9$ is due to the centrifugal force on the neutron, because it has spin-interaction with the two deuterons, and do not allow them to move toward the central $2 \mathrm{He} 4$.

Now consider that the neutron of 4Be9 captures a proton, and it transmutes to $5 \mathrm{~B} 10$, as seen in the Figure 26a. Then deuteron d-2 goes immedi- 
ately to left side, getting spin-interaction with $d-3$. But $d-1$ gets oscillatory motion toward the z-axis, as shown by the pink arrow. In that position $d-1$ does not get stability, because when it moves toward up direction, it has spin-interaction a little stronger with $d-2$ than with $d-3$. Besides, the flat structure formed by the three deuterons situated in the same plane is also unstable, because they have tendency to slip and to form a three-dimensional structure. Then finally $d-1$ goes to the left side, as seen in Figure 26b.

Note that 5B10 structure seen in Figure 26b has very big quadrupole moment $Q(B 10)$, because the charges of the three deuterons are all them situated in the same left side, with a big distance between $d-1$ and (d-2\&d-3). Also, we have to expect that $Q(B 10)$ for the structure in the Figure $26 \mathrm{~b}$ must be a little larger than $\mathrm{Q}(\mathrm{Be} 9)=+0.0529$. And this expectation is confirmed by experiments, the measured value is $Q(B 10)=+0.0845$ barns.

For the calculation of magnetic moment for the structure for 5B10, seen in the Figure 27, is used eq. (144) at the paper [4], ahead numbered as eq. (31). The value obtained is $+1.918 \mu \mathrm{N}$, and the experimental is $+1.801 \mu \mathrm{N}$.

$\mathrm{K}_{\mathrm{TH}}(\mathrm{X})=\left(\frac{\Phi}{6}\right) \cdot 1 \cdot 37176 \cdot \frac{\operatorname{PwR}(\mathrm{X}) \cdot \mathrm{R}(\mathrm{X})}{\operatorname{PwR}(015) \cdot \mathrm{R}(015)}$

Looking at the Figure 27, we realize why $Q(B 10)$ can be 4,2 times larger than $Q(N 14)$, because 7N14 has the charges of the protons distributed in the two sides around the central $2 \mathrm{He} 4$.

\section{Wrong Math Procedure used in Nuclear Physics for the Calculation of Magnetic Moments for Excited Even Z = N Nuclei}

Some excited $2+$ even-even nuclei have non-null magnetic moment, as for instance $10 \mathrm{Ne} 20,14 \mathrm{Si} 28$, 16S32. But there are some of them whose magnetic moment was not detected as non-null in the experiments. And as an excited even-even nucleus cannot have null magnetic moment, because that is impossible from the foundations of the Nuclear Physics, it was mandatory to develop some special techniques of measurements, with the aim to get non-null magnetic moments for them.

In 2001 was published the Atomic Data and Nuclear Data Tables, by Raman, et al. and the nuclear physicists are using it for the calculation of magnetic moment for excited $2+$ even-even nuclei, and their calculations are being successful in their attempts of getting values non-null for the magnetic moment of those excited even-even nuclei.

However, as shown in [17], the procedure for calculation of the transition probability in even $\mathrm{Z}=\mathrm{N}$ nuclei, from the ground to the first-excited $2+$ state, used in Raman's table, was invalidated in 2012, when new experimental findings [14] have shown to be wrong a dogma considered by nuclear theorists as untouchable: the even-even nuclei at the ground state are no spherical, as considered along decades. As consequence, Raman's table cannot be used for the calculation of the excited even-even nuclei, and thereby since 2012 were invalidated those theoretical attempts, with the aim to prove that there is not any excited $2+$ even-even nucleus with null magnetic moment. In another words, since 2001 the nuclear theorists were trying to save the Nuclear Physics from the total collapse, by using a wrong mathematical procedure (because at that time it was impossible to know that their procedure was wrong). In 2012 a new experiment proved that their attempt had failed, but they did not understand that, and continued using the Raman's table for the excited even-even nuclei. Only in 2018 the author of the present paper discovered that their procedure is wrong.

Being invalidated the theoretical attempts with the aim to reach values non-null for the magnetic moment of some excited $2+$ even-even nuclei (by using a correct procedure of calculation), the situation nowadays is that there are, indeed, some excited $2+$ even-even nuclei with null magnetic moment. And as this is impossible, by considering the foundations of the Nuclear Physics, this implies in the breakdown of the fundamental pillars on which the theory was developed. New foundations are required.

\section{Conclusions}

Since the beginning of the development of the Standard Nuclear Physics, some experimental findings were pointing out that something is wrong with the way adopted for the development of the theory. But along the years the nuclear theorists have neglected them, supported by that sort of philosophy according to which the theorists never have the final answer, since the advancement of science occurs step by step, by one unsatisfactory 
theory being replaced by a better one. Besides, in spite of the nuclear theorists realized that it is impossible to find a unique nuclear model from the foundations of nuclear physics, in order that, from such a unique model, all the nuclear properties of atomic nuclei could be calculated in good agreement with the data obtained from experiments, no matter how much uncomfortable was their disappointment of being obliged to develop a theory by using several nuclear models, however they had at hand some palliative justification for that undesirable failure, as to quote Bohr's Complementarity Principle, or to claim that from Nuclear Theory the advancement of technology was exceptional.

But along the current beginning of the $21^{\text {th }}$ Century the situation was changed, because the foundations of the Standard Nuclear Physics have been losing credibility face to several experimental findings, denying them, as for instance the discovery that even-even nuclei at the ground state have ellipsoidal shape, and this is impossible from its foundations. Besides, Bohr's Complementarity experienced a hard blow [18], invalidating the philosophical viewpoint that physical phenomena require different structures to produce them.

As less paradoxes a theory has, as better it is. If Nuclear Physics was established on correct foundations, we had to expect that, taking in consideration the claim that the evolution of science occurs steps by steps, by one unsatisfactory model being replaced by a new one, the advancement of Nuclear Physics along the years should be occurring in parallel to the gradual elimination of the paradoxes. But what we see nowadays is just the contrary. Year after year the quantity of paradoxes increases, and what is worst, the new paradoxes of the $21^{\text {th }}$ Century are appearing in the pillars of the theory, demolishing them, one by one, day after day. The quantity of paradoxes also grows because some nuclear theorists try to explain a paradox by introducing a new paradox, and so, instead of one paradox, the initial paradox is converted to two paradoxes. For instance, some theorists try to explain the Gamow's paradox by claiming that "the kinetic energy is negative in the forbidden region, so the velocity is formally an imaginary number". We could accept such nonsense if we had any idea of what a hell can be a velocity formally described by an imaginary number. So, when the theorists introduce a new paradox, in their attempt of trying to solve an old paradox, and the new paradox is born because a dirty math was applied, we have clear reasons to conclude that we must have a strong distrust about that theory. Using a dirty math we may confirm any absurd we wish.

So, face to the catastrophic situation of the Nuclear Physics, finally we have to ask to ourselves. "How many pillars have to be demolished, so that the nuclear theorists finally realize that they have a theory without foundations, since the foundations were invalidated by experiments.

\section{References}

1. M Urban, F Couchot, X Sarazin, A Djannati-Atai (2013) The quantum vacuum as the origin of the speed of light, European Physical Journal D.

2. Guglinski W (2006) Quantum Ring Theory, Bäuu Institute Press. Boulder, Co, USA.

3. Guglinski W (2018) Calculation of magnetic moments of light nuclei with number of protons between $Z=3$ and $Z=30$. SF J Nuclear Science 1: 1 .

4. Guglinski W (2018) Testing the equations of the new nuclear model of hexagonal floors. SF J Nuclear Science 2: 4.

5. W Guglinski (2018) Mathematical confirmation for the nuclear properties $\mathrm{K}(\mathrm{O})=\mathrm{K}(\mathrm{Ca})=1 / 2 . \mathrm{K}(\mathrm{Si})=$ $1 / 2 . \mathrm{K}(\mathrm{Fe})$. SF J Nuclear Science 2: 4.

6. Eisberg R, Resnick R (1974) Quantum, solids, physics of atoms, molecules nuclei and particles. John Wiley \& Sons, Inc.

7. Guglinski W (2018) On how Bohr model of hydrogen atom is connected to nuclear physics. IJFPS 8: 44-53.

8. Guglinski W (2018) Demonstration of the new Coulomb's Law $F=K Q q / d X, X<2$, for distances $d$ between 10-15m and 10-12m. SF J Nuclear Science 2: 5.

9. A Krieger, K Blaum, ML Bissell, N Frömmgen, Ch Geppert, et al. (2012) Nuclear Charge Radius of 12Be. Physical Review Letters 108: 14.

10.Nörtershäuser $W$, Tiedemann $D$, Záková $M$, Andjelkovic Z, Blaum K, et al. (2009) Nuclear Charge Radii of $7,9,10 \mathrm{Be}$ and the One-Neutron Halo Nucleus 11Be. Phys Rev Lett 102: 062503.

11.Gaffney LP, Butler PA, Scheck M, Hayes AB, Wenander F, et al. (2013) Studies of pear-shaped nuclei using accelerated radioactive beams. Nature 497: 199-204.

12.KA Azzam, MA Fawzy, EM Hassan, AM Yasser (2005) Electron-deuteron tensor polarization and D-State probability. Turk J Phys 29: 127-135. 
13.Guglinski W (2018) Re-evaluation of Fermi's theory of beta-decay. IJFPS 8: 19-43.

14.Ebran JP, Khan E, Nikšić T, Vretenar D (2012) How atomic nuclei cluster. Nature 487: 341-344.

15. Hessenfelder S (2018) Lost in Math, how beayty leads physics astray. Basic Books. New York, NY, USA.

16. NH Frank (1937) Note on the Hartree and HartreeFock Methods. Phys Rev 51: 577.
17.Guglinski W (2019) Wrong math procedure used in nuclear physics for the calculation of magnetic moments of excited Z5N even-even nuclei. Physics Essays 32: 307-312.

18.Rabinowitz M (2012) Challenges to Bohr's WaveParticle Complementarity Principle. International Journal of Theoretical Physics 52: 668-678. 